\title{
Stratifying Lie Strata of Hilbert Modular Varieties
}

\author{
Chia-Fu Yu, Ching-Li Chai* and Frans Oort
}

\begin{abstract}
In this survey we explain a stratification of a Hilbert modular variety $\mathscr{M}_{E}$ in characteristic $p>0$ attached to a totally real number field $E$. This stratification refines the stratification of $\mathscr{M}_{E}$ by Lie type, and has the property that many strata are central leaves in $\mathscr{M}_{E}$, called distinguished central leaves.

In the case when the totally real field $E$ is unramified above $p$, this stratification reduces to the stratification of $\mathscr{M}_{E}$ by $\alpha$-type first introduced by Goren and Oort and studied by $\mathrm{Yu}$, and coincides with the EO stratification on $\mathscr{M}_{E}$. Moreover it is known that every non-supersingular $\alpha$-stratum of $\mathscr{M}_{E}$ is irreducible. To treat the general case where $E$ may be ramified above $p$, a key ingredient is the notion of congruity, a $p$-adic numerical invariant for abelian varieties with real multiplication by $\mathcal{O}_{E}$ in characteristic $p$. For every Lie stratum $\mathcal{N}_{\underline{e}}$ on $\mathscr{M}_{E}$, this new invariant defines a finite number of locally closed subsets $\mathcal{Q}_{\underline{c}}\left(\mathcal{N}_{\underline{e}}\right)$, and $\mathcal{N}_{\underline{e}}$ is the disjoint union of these Liecongruity strata $\mathcal{Q}_{\underline{c}}\left(\mathcal{N}_{\underline{e}}\right)$ in $\mathcal{N}_{\underline{e}}$.

The incidence relation between the Lie-congruity strata enables one to show that the prime-to- $p$ Hecke correspondences operate transitively on the set of all irreducible components of any distinguished central leaf in $\mathscr{M}_{E}$, see Theorems 7.1, 8.1 and 9.1. The Hecke transitivity implies, according to the method of prime-to- $p$ monodromy of Hecke invariant subvarieties, that every non-supersingular distinguished central leaf in a Hilbert modular variety $\mathscr{M}_{E}$ is irreducible. The last irreducibility result is a key ingredient of the proof the Hecke orbit conjecture for Siegel modular varieties.
\end{abstract}

\section{Introduction}

Moduli spaces in characteristic $p>0$ have natural stratifications coming from $p$-adic invariants of the geometric objects they classify. For instance, the moduli space $\mathscr{A}_{g, 1, n}$ over $\overline{\mathbb{F}_{p}}$ which classifies $g$-dimensional principally polarized abelian varieties in characteristic $p$ with symplectic level- $n$ structure has several stratifications; e.g., the stratifications by the Newton polygon (respectively the $p$-rank, respectively the $a$-number) of an abelian variety. These stratifications are helpful in understanding the geometry and arithmetic of moduli spaces.

Received November 25, 2019; Accepted March 29, 2020.

Communicated by Jenn-Nan Wang.

2010 Mathematics Subject Classification. 14G35, $14 \mathrm{~K} 10$.

Key words and phrases. Hilbert modular varieties, Hecke orbits, stratifications.

*Corresponding author. 
This article is a survey of the congruity stratification of Lie strata in a Hilbert modular variety $\mathscr{M}_{E}$ over $\overline{\mathbb{F}_{p}}$ attached to a totally real number field $E$. We give complete definitions and statements of results but no proofs, so "summary of results" is perhaps more befitting than "survey". Detailed proofs can be found in [9, Chapter 4]. We have included a long introduction, together with preliminary materials in Sections 24, to make this article more accessible. The actual summary itself in Sections 5 9, which inevitably is quite technical, occupies about a third of the total number of pages. The last Section 10 , consists of a list of related questions.

Throughout this paper $p$ is a fixed prime number, $E$ is a totally real number field.

1.1. Motivation: the Hecke orbit conjecture for Siegel modular varieties

This paper was motivated by the Hecke orbit problem for the Siegel modular variety $\mathscr{A}_{g, 1, n}$ over $\overline{\mathbb{F}_{p}}$, with $n \geq 3$ and $\operatorname{gcd}(p, n)=1$. For every $\overline{\mathbb{F}_{p}}$-point $x$ of $\mathscr{A}_{g, 1, n}$ which corresponds to a principally polarized abelian variety with symplectic level- $n$ structure $\left(A_{x}, \lambda_{x}, \eta_{x}\right)$, the central leaf $\mathcal{C}_{\mathscr{A}_{g, 1, n}}(x)$ is the locally closed smooth subscheme of $\mathscr{A}_{g, 1, n}$ over $\overline{\mathbb{F}_{p}}$, characterized by

$$
\mathcal{C}_{\mathscr{A}_{g, 1, n}}(x)\left(\overline{\mathbb{F}_{p}}\right)=\left\{y \in \mathscr{A}_{g, 1, n}\left(\overline{\mathbb{F}_{p}}\right) \mid\left(A_{y}, \lambda_{y}\right)\left[p^{\infty}\right] \cong\left(A_{x}, \lambda_{x}\right)\left[p^{\infty}\right]\right\}
$$

see [30. In other words for every geometric point $y \in \mathscr{A}_{g, 1, n}\left(\overline{\mathbb{F}_{p}}\right), y$ lies in the central leaf $\mathcal{C}_{\mathscr{A}_{g, 1, n}}(x)$ if and only if the principally polarized $p$-divisible group $\left(A_{y}, \lambda_{y}\right)\left[p^{\infty}\right]$ attached to $y$ is isomorphic to $\left(A_{x}, \lambda_{x}\right)\left[p^{\infty}\right]$.

On the other hand, the prime-to- $p$ Hecke orbit of $x$ is the countable subset $\mathcal{H}_{\mathrm{Sp}_{2 g}\left(\mathbb{A}_{f}^{(p)}\right)}(x)$ of $\mathscr{A}_{g, 1, n}\left(\overline{\mathbb{F}_{p}}\right)$, consisting of all points $\left[\left(A_{z}, \lambda_{z}, \eta_{z}\right)\right] \in \mathscr{A}_{g, 1, n}\left(\overline{\mathbb{F}_{p}}\right)$ such that there exists a prime-to- $p$ quasi-isogeny $\alpha: A_{z} \rightarrow A_{x}$ such that $\alpha^{*}\left(\lambda_{x}\right)=\lambda_{z}$ and $\alpha^{*}\left(\eta_{z}\right)=\eta_{x}$.

The Hecke orbit conjecture for $\mathscr{A}_{g, 1, n}$ predicts that the prime-to-p Hecke orbit $\mathcal{H}_{\mathrm{Sp}_{2 g}\left(\mathbb{A}_{f}^{(p)}\right)}(x)$ is Zariski dense in the central leaf $\mathcal{C}_{\mathscr{A}_{g, 1, n}}(x)$.

A complete proof will appear in [9, Chapter 8]; see also [5], [9, 8.1], 11 for outlines of the proof. Below is a brief description of how Hilbert modular varieties enter the proof.

A starting point of the proof of the Hecke orbit conjecture for Siegel modular varieties is Tate's theorem that every abelian variety over a finite field admits sufficiently many complex multiplications. This implies the existence of a Hecke-equivariant correspondence from a product of Hilbert modular varieties to the Siegel modular variety, called the "Hilbert trick".

For every $x \in \mathscr{A}_{g, 1, n}\left(\overline{\mathbb{F}_{p}}\right)$, there exists a product of totally real number fields $E_{1}, \ldots, E_{r}$ with $\sum_{i=1}^{r}\left[E_{i}: \mathbb{Q}\right]=g$, central leaves $\mathcal{C}_{\mathscr{M}_{E_{i}}}\left(y_{i}\right)$ in Hilbert mod- 
ular varieties $\mathscr{M}_{E_{i}}, i=1, \ldots, r$, and a finite-to-one Hecke-equivariant correspondence $f: \mathcal{C}_{\mathscr{M}_{E_{1}}}\left(y_{1}\right) \times \cdots \times \mathcal{C}_{\mathscr{M}_{E_{r}}}\left(y_{r}\right) \rightarrow \mathscr{A}_{g, 1, n}$, such that the image under $f$ of an irreducible component of $\mathcal{C}_{\mathscr{M}_{E_{1}}}\left(y_{1}\right) \times \cdots \times \mathcal{C}_{\mathscr{M}_{E_{r}}}\left(y_{r}\right)$ is in finite isogeny correspondence with a locally closed subvariety of the Zariski closure of $\mathcal{H}_{\mathrm{Sp}_{2 g}\left(\mathbb{A}_{f}^{(p)}\right)}(x)$ in $\mathcal{C}_{\mathscr{A}_{g, 1, n}}(x)$.

In the above statement we have used the fact that the Zariski closure in $\mathcal{C}_{\mathscr{M}_{E_{i}}}\left(y_{i}\right)$ of the prime-to- $p$ Hecke orbit of $y_{i}$, a countable subset of $\mathcal{C}_{\mathscr{M}_{E_{i}}}\left(y_{i}\right)$ is both open and closed in the central leaf $\mathcal{C}_{\mathscr{M}_{E_{i}}}\left(y_{i}\right)$. See Subsections 1.2 and 1.3 for the definition of Hilbert modular varieties $\mathscr{M}_{E_{i}}$ and central leaves in them.

With the help of the action of the local stabilizer subgroup at a supersingular point of the Zariski closure of the Hecke orbit $\mathcal{H}_{\mathrm{Sp}_{2 g}\left(\mathbb{A}_{f}^{(p)}\right)}(x)$ in $\mathscr{A}_{g, 1, n}$, we may further assume that there is only one prime above $p$ in $\mathcal{O}_{E_{i}}$ for each $i=1, \ldots, r$.

Changing the $y_{i}$ 's by $E_{i}$-isogeny, we may also assume that each of the central leaves $\mathcal{C}_{\mathscr{M}_{E_{i}}}\left(y_{i}\right)$ in $\mathscr{M}_{E_{i}}$ is distinguished; see Subsection 1.7. The critical information which comes from the congruity stratification of the Lie strata is Corollary 9.4

Every non-supersingular distinguished central leaf in $\mathscr{M}_{E_{i}}$ is irreducible.

It is known that every central leaf in $\mathscr{M}_{E_{i}}$ contains a robust hypersymmetric point which corresponds to an abelian variety $B_{i}$ over $\overline{\mathbb{F}_{p}}$ with at most two slopes, such that the natural $\operatorname{map} \operatorname{End}\left(B_{i}\right) \otimes_{\mathbb{Z}} \mathbb{Q}_{p} \rightarrow \operatorname{End}\left(B_{i}\left[p^{\infty}\right]\right) \otimes_{\mathbb{Z}_{p}} \mathbb{Q}_{p}$ is an isomorphism. So we conclude that the Zariski closure in $\mathcal{C}_{\mathscr{A}_{g, 1, n}}(x)$ of the prime-to- $p$ Hecke orbit $\mathcal{H}_{\mathrm{Sp}_{2 g}\left(\mathbb{A}_{f}^{(p)}\right)}(x)$ contains a robust hypersymmetric point; cf. 9, Chapter 8] and 10 .

With a hypersymmetric point at hand, we are in a position to apply the local rigidity of $p$-divisible groups [7], and the density of $\mathcal{H}_{\operatorname{Sp}_{2 g}\left(\mathbb{A}_{f}^{(p)}\right)}(x)$ in $\mathcal{C}_{\mathscr{A}_{g, 1, n}}(x)$ follows.

To recapitulate: the irreducibility of non-supersingular distinguished central leaves in a Hilbert modular variety $\mathscr{M}_{E}$ implies the existence of a robust hypersymmetric point in the Zariski closure of a Hecke orbit $\mathcal{H}_{\mathrm{Sp}_{2 g}\left(\mathbb{A}_{f}^{(p)}\right)}(x)$ in the central leaf $\mathcal{C}_{\mathscr{A}_{g, 1, n}}(x)$. This irreducibility result is non-trivial - we don't know of any proof by "pure thought".

In Subsections 1.21 .8 below we give a very brief introduction to Lie strata and Liecongruity strata on Hilbert modular varieties, as a tour map for Sections 29.

\subsection{Hilbert modular varieties}

Given an element $[\widetilde{\mathscr{L}}]$ of the strict class group $\widetilde{\mathrm{Cl}} l_{E}$ of $E$, a positive integer $n \geq 3$ prime to $p$, and a generator $\bar{\delta}$ of the free rank-one $\left(\mathcal{O}_{E} / n \mathcal{O}_{E}\right)$-module

$$
\left(\mathscr{L}^{\vee} \otimes_{\mathcal{O}_{E}} \mathcal{D}_{E / \mathbb{Q}}^{-1} / n \mathscr{L}^{\vee} \otimes_{\mathcal{O}_{E}} \mathcal{D}_{E / \mathbb{Q}}^{-1}\right) \otimes_{(\mathbb{Z} / n \mathbb{Z})} \mu_{n}\left(\overline{\mathbb{F}_{p}}\right)
$$


we have an associated Hilbert modular variety $\mathscr{M}_{E, n, \bar{\delta}, \overline{\mathbb{F}_{p}}}^{\widetilde{L}}$ over $\overline{\mathbb{F}_{p}}$, which classifies principally $\widetilde{\mathscr{L}}$-polarized abelian schemes of relative dimension $g$ over $\overline{\mathbb{F}_{p}}$-schemes, with real multiplication by $\mathcal{O}_{E}$ with symplectic level- $n$ structures. See Subsection 2.1, Definitions 2.2, 2.3 and 2.4 for the precise definitions.

The Hilbert modular variety $\mathscr{M}_{E, n, \bar{\delta}, \overline{\mathbb{F}_{p}}}^{\widetilde{\mathscr{L}}}$ is a reduced irreducible normal $g$-dimensional scheme over $\overline{\mathbb{F}_{p}}$, and is a local complete intersection. It is smooth over $\overline{\mathbb{F}_{p}}$ if and only if $E$ is unramified above $p$. When $E$ is ramified above $p$, the locus in $\mathscr{M}_{E, n, \bar{\delta}, \overline{\mathbb{F}_{p}}}^{\widetilde{\mathscr{L}}}$ consisting of singular points is a closed subset of $\mathscr{M}_{E, n, \overline{\mathscr{L}}, \overline{\mathbb{F}_{p}}}^{\widetilde{C}}$ all of whose irreducible components have codimension 2 in $\mathscr{M}_{E, n, \bar{\delta}, \overline{\mathbb{F}_{p}}}^{\widetilde{\mathscr{L}}}$.

Since geometric properties of the modular variety $\mathscr{M}_{E, n, \bar{\delta}, \overline{\mathbb{F}_{p}}}^{\widetilde{\mathscr{L}}}$ are all independent of the parameters $(\widetilde{\mathscr{L}}, n, \bar{\delta})$, we will shorten $\mathscr{M}_{E, n, \bar{\delta}, \overline{\mathbb{F}_{p}}}^{\widetilde{\mathscr{L}}}$ to $\mathscr{M}_{E}$ if no confusion is likely.

Remark. Strictly speaking the Hilbert modular variety $\mathscr{M}_{E, n, \bar{\delta}, \overline{\mathbb{F}_{p}}}^{\widetilde{\mathscr{L}}}$ are not a special cases of PEL type modular varieties as defined in 23 unless the element $[\widetilde{\mathscr{L}}]$ is 0 in the strict class group $\widetilde{\mathrm{Cl}} l_{E}$. Still, the modular varieties $\mathscr{M}_{E, n, \overline{\mathcal{L}}, \overline{\mathbb{F}_{p}}}^{\widetilde{\mathscr{S}}}$ should be regarded as analogs of the Siegel moduli space $\mathscr{A}_{g, 1, n}$ of $g$-dimensional principally polarized abelian varieties with level- $n$ structures; cf. Remark 2.2.3.

We will not discuss those moduli spaces which classify $g$-dimensional $\mathcal{O}_{E}$-linear abelian varieties in characteristic $p$ together with $\mathcal{O}_{E}$-linear polarizations of some fixed degree which is divisible by $p$.

\subsection{Central leaves in $\mathscr{M}_{E}$}

For an $\overline{\mathbb{F}_{p}}$-point $x$ of a Hilbert modular variety $\mathscr{M}_{E}$ which corresponds to an abelian variety with real multiplication by $\mathcal{O}_{E}$ with level- $n$-structure $\left(A_{x}, \lambda_{x}, \iota_{x}, \eta_{x}\right)$, the central leaf in $\mathscr{M}_{E}$ passing through $x$ is, by definition, the locally closed smooth subvariety of $\mathscr{M}_{E}$ over $\overline{\mathbb{F}_{p}}$ such that

$$
\mathcal{C}_{\mathscr{M}_{E}}(x)\left(\overline{\mathbb{F}_{p}}\right)=\left\{y \in \mathscr{M}_{E}\left(\overline{\mathbb{F}_{p}}\right) \mid\left(A_{y}, \lambda_{y}, \iota_{y}\right)\left[p^{\infty}\right] \cong\left(A_{x}, \lambda_{x}, \iota_{x}\right)\left[p^{\infty}\right]\right\}
$$

Certain central leaves in Hilbert modular varieties $\mathscr{M}_{E}$, called distinguished central leaves, are irreducible. Every distinguished central leaf in $\mathscr{M}_{E}$ is a Lie-congruity stratum of $\mathscr{M}_{E}$. See Subsection 1.6 for a short description of Lie-congruity strata, and Definitions 6.2.1 and 6.3.5 for the definition of distinguished central leaves. Every central leaf in $\mathscr{M}_{E}$ is in a finite isogeny correspondence with a distinguished central leaf. As already mentioned, the proof of the Hecke orbit conjecture in [9, Chapter 8] depends heavily on the fact that every non-supersingular distinguished central leaf in $\mathscr{M}_{E}$ is irreducible. 


\subsection{The Lie stratification of $\mathscr{M}_{E}$}

Deligne and Pappas [13] introduced a stratification of $\mathscr{M}_{E}$ by the isomorphism classes of $\operatorname{Lie}\left(A_{x}\right)$ as vector spaces with actions by $\mathcal{O}_{E} / p \mathcal{O}_{E}$, where $x$ runs through all geometric points of $\mathscr{M}_{E}$, and $\operatorname{Lie}\left(A_{x}\right)$ is the Lie algebra of the fiber at $x$ of the universal abelian scheme with real multiplication by $\mathcal{O}_{E}$ over $\mathscr{M}_{E}$. Under this stratification by Lie type, or the Lie stratification of $\mathscr{M}_{E}$, the set underlying $\mathscr{M}_{E}$ is the disjoint union of Lie strata: $\mathscr{M}_{E}=\bigsqcup_{\underline{e} \in \mathcal{T}_{E}^{\text {sd }}} \mathcal{N}_{\underline{e}}$. Here $\mathcal{N}_{\underline{e}}$ is the locally closed subset of $\mathscr{M}_{E}$ such that a point $x$ of $\mathscr{M}_{E}$ is in $\mathcal{N}_{\underline{e}}$ if and only if the isomorphism class of the $\left(\mathcal{O}_{E} / p \mathcal{O}_{E}\right)$-module $\operatorname{Lie}\left(A_{x}\right)$ corresponds to the element $\underline{e}$ of the indexing set $\mathcal{T}_{E}^{\text {sd }}$. Moreover each Lie stratum $\mathcal{N}_{\underline{e}}$ with the reduced scheme structure is a smooth locally closed subscheme of $\mathscr{M}_{E}$, and the Zariski closure of $\mathcal{N}_{\underline{e}}$ is a local complete intersection and is normal.

The indexing set $\mathcal{T}_{E}^{\text {sd }}$ for the Lie stratification of $\mathscr{M}_{E}$ has a natural structure as a finite poset (partially ordered set). The poset $\mathcal{T}_{E}^{\text {sd }}$ is ranked, in the sense that all maximal chains between any two elements of $\mathcal{T}_{E}^{\text {sd }}$ have the same length. Moreover it has a unique maximal element and also a unique minimal element. This partial ordering on $\mathcal{T}_{E}^{\mathrm{sd}}$ is compatible with the incidence relation of Lie strata, in the following sense: for any two elements $\underline{e}_{1}, \underline{e}_{2} \in \mathcal{T}_{E}^{\text {sd }}$, the Lie stratum $\mathcal{N}_{\underline{e}_{1}}$ is contained in the Zariski closure of $\mathcal{N}_{\underline{e}_{2}}$ if and only if $\underline{e}_{1} \preceq \underline{e}_{2}$. The Lie stratum corresponding to the maximal element in $\mathcal{T}_{E}^{\text {sd }}$ is the smooth locus $\mathscr{M}_{E}^{\mathrm{sm}}$, consisting of all smooth points of $\mathscr{M}_{E}$. The dimension of every irreducible component of the minimal Lie stratum $\mathcal{N}_{\text {min }}$, indexed by the minimal element $\underline{e}_{E \text {,min }}^{\text {sd }}$ of $\mathcal{T}_{E}^{\text {sd }}$, is $\sum_{e_{v} \text { odd }} f_{v}$. The sum here runs through all places $v$ of $E$ above $p$ whose ramification index $e_{v}$ is odd, and $f_{v}:=\left[\kappa_{v}: \mathbb{F}_{p}\right]$, the degree of the residue field $\kappa_{v}$ of $\mathcal{O}_{E_{v}}$.

\subsection{The $\alpha$-stratification of $\mathscr{M}_{E}$ when $E$ is unramified above $p$}

In this subsection we assume that the totally real field $E$ is unramified above $p$. In this case the poset $\mathcal{T}_{E}^{\text {sd }}$ is a singleton, the Hilbert modular variety $\mathscr{M}_{E}$ over $\overline{\mathbb{F}_{p}}$ is smooth, and the Lie stratification of $\mathscr{M}_{E}$ is trivial.

(i) The stratification of the Hilbert modular variety $\mathscr{M}_{E}$ by the isomorphism classes of the maximal $\alpha$-subgroup scheme of $A_{x}[p]$ with action by $\mathcal{O}_{E} / p \mathcal{O}_{E}$ attached to geometric points $\left[\left(A_{x}, \lambda_{x}, \iota_{x}, \eta_{x}\right)\right]$ of $\mathscr{M}_{E}$ was studied in [14], called the $\alpha$-stratification.

(ii) One of the main results of [14] says that if $k \supseteq \mathbb{F}_{p}$ is an algebraically closed field and $x, y$ are $k$-points of $\mathscr{M}_{E}$ such that the maximal $\left(\mathcal{O}_{E} / p \mathcal{O}_{E}\right)$-linear $\alpha$-subgroup schemes of $A_{x}[p]$ and $A_{y}[p]$ are isomorphic, then there exists an $\left(\mathcal{O}_{E} / p \mathcal{O}_{E}\right)$-linear isomorphism from $A_{x}[p]$ to $A_{y}[p]$ which respects the principal polarizations. In other words, the stratification of $\mathscr{M}_{E}$ by $\alpha$-types is a natural generalization of the EO stratification of Siegel modular varieties $\mathscr{A}_{g, 1, n}$ defined in 29 . 
(iii) The $\alpha$-stratification of $\mathscr{M}_{E}$ has a unique dense open stratum, which coincides with the ordinary locus $\mathscr{M}_{E}^{\text {ord }}$ in $\mathscr{M}_{E}$, i.e., the open subset $\mathscr{M}_{E}^{\text {ord }}$ of $\mathscr{M}_{E}$ whose points correspond to ordinary abelian varieties. The complement of $\mathscr{M}_{E}^{\text {ord }}$ in $\mathscr{M}_{E}$ is a smooth divisor $D$ with normal crossings, and the $\alpha$-stratification coincides with the stratification of $\mathscr{M}_{E}$ associated to the divisor $D$. Moreover, some of the $\alpha$-strata are actually central leaves in $\mathscr{M}_{E}$.

The congruity stratification of Lie strata of Hilbert modular varieties $\mathscr{M}_{E}$ for possibly ramified totally real fields $E$, described in Subsections 1.61 .8 below, extends the $\alpha$-stratification for Hilbert modular varieties attached to totally real fields unramified above $p$ considered in this subsection: if $E$ is unramified above $p$, then $\mathscr{M}_{E}$ has a single Lie stratum, and the congruity stratification of this unique Lie stratum coincides with the $\alpha$-stratification.

\subsection{The congruity stratification of Lie strata of $\mathscr{M}_{E}$}

A numerical invariant $\underline{c}\left(A_{x}\right)$ with values in a finite poset $\boldsymbol{\tau}_{E}$, for $g$-dimensional principally $\widetilde{\mathscr{L}}$-polarized abelian varieties $\left(A_{x}, \lambda_{x}, \iota_{x}\right)$ with real multiplication by $\mathcal{O}_{E}$ attached to points $x$ of $\mathscr{M}_{E}$, was defined in [40, 2.4]. This invariant, called congruity in [9, Chapter 4], is a refinement of the $\alpha$-type of $A_{x}$, the invariant which defines the $\alpha$-stratification. The invariant $\underline{c}\left(A_{x}\right)$ gives rise to a stratification on every Lie stratum $\mathcal{N}_{\underline{e}}$ of $\mathscr{M}_{E}$ : we have $\mathcal{N}_{\underline{e}}=\bigsqcup_{\underline{c}} \mathcal{Q}_{\underline{c}}\left(\mathcal{N}_{\underline{e}}\right)$, where $\mathcal{Q}_{\underline{c}}\left(\mathcal{N}_{\underline{e}}\right)$ is the locally closed subset of $\mathcal{N}_{\underline{e}}$, consisting of points of $\mathcal{N}_{\underline{e}}$ whose congruity is $\underline{c}$. Below are some properties of the stratification of $\mathcal{N}_{\underline{e}}$ by congruity, for every $\underline{e} \in \mathcal{T}_{E}^{\text {sd }}$.

(i) The finite poset $\boldsymbol{\tau}_{E}$ is ranked, and has a unique maximal element, denoted by $\underline{0}$.

(ii) Each stratum $\mathcal{Q}_{\underline{c}}\left(\mathcal{N}_{\underline{e}}\right)$ is a smooth locally closed subscheme of $\mathcal{N}_{\underline{e}}$.

(iii) The Zariski closure in $\mathcal{N}_{\underline{e}}$ of $\mathcal{Q}_{\underline{c}}\left(\mathcal{N}_{\underline{e}}\right)$, is equal to $\mathcal{Q}_{\preceq \underline{c}}\left(\mathcal{N}_{\underline{e}}\right):=\bigsqcup_{\underline{\underline{c}^{\prime}} \preceq \underline{c}} \mathcal{Q}_{\underline{c}^{\prime}}\left(\mathcal{N}_{\underline{e}}\right)$, which is smooth over $\overline{\mathbb{F}_{p}}$, for every $\underline{c} \in \boldsymbol{\tau}_{E}$.

(iv) The stratum $\mathcal{Q}_{\underline{0}}\left(\mathcal{N}_{\underline{e}}\right)$ is a dense open subscheme of $\mathcal{N}_{\underline{e}}$.

(v) The complement of $\mathcal{Q}_{\underline{0}}\left(\mathcal{N}_{\underline{e}}\right)$ in $\mathcal{N}_{\underline{e}}$, if non-empty, is a divisor in $\mathcal{N}_{\underline{e}}$ with normal crossings.

We call a subset of $\mathscr{M}_{E}$ of the form $\mathcal{Q}_{\underline{c}}\left(\mathcal{N}_{\underline{e}}\right)$ a Lie-congruity stratum of $\mathscr{M}_{E}$.

A warning is in order, that the incidence relation between Lie-congruity strata is not well understood yet. For instance we don't know whether the Zariski closure $\left(\mathcal{Q}_{\underline{c}}\left(\mathcal{N}_{\underline{e}}\right)\right)^{\text {zar }}$ in $\mathscr{M}_{E}$ of $\mathcal{Q}_{\underline{c}}\left(\mathcal{N}_{\underline{e}}\right)$ is a union of Lie-congruity strata, for every Lie-congruity stratum $\mathcal{Q}_{\underline{c}}\left(\mathcal{N}_{\underline{e}}\right) ;$ see Question 10.1 . 
The Lie type and the congruity of an $\widetilde{\mathscr{L}}$-polarized abelian variety $\left(A_{x}, \lambda_{x}, \iota_{x}\right)$ in characteristic $p$ depend only on the polarized $\mathcal{O}_{E}$-linear $p$-divisible group $\left(A_{x}\left[p^{\infty}\right], \lambda_{x}\left[p^{\infty}\right], \iota_{x}\left[p^{\infty}\right]\right)$ associated to $\left(A_{x}, \lambda_{x}, \iota_{x}\right)$. Since the isomorphism class of $\left(A_{x}\left[p^{\infty}\right], \lambda_{x}\left[p^{\infty}\right], \iota_{x}\left[p^{\infty}\right]\right)$ does not change under prime-to- $p$ Hecke correspondences associated to $\mathrm{SL}_{2}\left(\mathbb{A}_{f}^{(p)}\right)$, every Liecongruity stratum $\mathcal{Q}_{\underline{c}}\left(\mathcal{N}_{\underline{e}}\right)$ is stable under all prime-to- $p$ Hecke correspondences on $\mathscr{M}_{E}$. More information on prime-to- $p$ Hecke symmetries on Siegel and Hilbert modular varieties in characteristic $p$ can be found in [4, 42].

\subsection{Distinguished Lie-congruity strata}

We will define a subset of the set of all Lie-congruity strata of $\mathscr{M}_{E}$, called distinguished Lie-congruity strata; see Definition 5.6. They have two useful properties:

(a) For any algebraically closed field $k \supseteq \mathbb{F}_{p}$ and any two $k$-points $x_{1}, x_{2}$ on a distinguished Lie-congruity stratum, there exists an $\mathcal{O}_{E}$-linear isomorphism from $A_{x_{1}}\left[p^{\infty}\right]$ to $A_{x_{2}}\left[p^{\infty}\right]$ which respects the principal $\mathcal{O}_{E^{-}}$-linear polarizations induced by $\lambda_{x_{1}}$ and $\lambda_{x_{2}}$.

(b) For any algebraically closed field $k \supseteq \mathbb{F}_{p}$ and any $k$-point $x \in \mathscr{M}_{E}(k)$, there exists a $k$-point $y$ in a distinguished Lie-congruity stratum, and an $E$-linear quasi-isogeny from $A_{y}\left[p^{\infty}\right]$ to $A_{x}\left[p^{\infty}\right]$ which respects the principal $\mathcal{O}_{E}$-linear polarizations.

Property (a) says that each distinguished Lie-congruity stratum is a central leaf of the Hilbert modular variety $\mathscr{M}_{E}$, consisting of all points in $\mathscr{M}_{E}$ with a fixed geometric isomorphism class of principally polarized $\mathcal{O}_{E} \otimes_{\mathbb{Z}} \mathbb{Z}_{p}$-linear $p$-divisible groups; these central leaves are said to be distinguished. Property (b) implies that every central leaf on $\mathscr{M}_{E}$ is in isogeny correspondence with a distinguished central leaf.

\subsection{Specialization from distinguished central leaves to $E$-minimal points}

A key result on the geometry of distinguished central leaves on a Hilbert modular variety $\mathscr{M}_{E}$ is Theorem 9.1, which asserts that the Zariski closure of every irreducible component of a distinguished Lie-congruity stratum on $\mathscr{M}_{E}$ contains an E-minimal point, i.e., an $\overline{\mathbb{F}_{p}}$-point $x_{0} \in \mathcal{N}_{\min }\left(\overline{\mathbb{F}_{p}}\right)$ whose underlying abelian variety $A_{x_{0}}$ is superspecial. In other words $A_{x_{0}}$ is isomorphic to the product of $[E: \mathbb{Q}]$ supersingular elliptic curves over $\overline{\mathbb{F}_{p}}$.

Theorem 9.1 follows from the combination of Theorems 7.1 and 8.1. Theorem 7.1 says that the Zariski closure of every irreducible component of a distinguished Lie-congruity stratum which is not contained in the minimal Lie stratum $\mathcal{N}_{\text {min }}$ contains an irreducible component of a distinguished congruity stratum in $\mathcal{N}_{\text {min }}$. Its proof is intricate; see Subsection 7.4 for an impressionistic sketch. Theorem 8.1 says that the Zariski closure of every 
irreducible component of a congruity stratum in the minimal Lie stratum $\mathcal{N}_{\text {min }}$ contains an E-minimal point.

A consequence of Theorem 9.1 is the fact that the prime-to- $p$ Hecke correspondences operate transitively on the set of all irreducible components of any distinguished central leaf $\mathcal{Q}_{\underline{c}}\left(\mathcal{N}_{\underline{e}}\right)$ of $\mathscr{M}_{E}$; see Corollary 9.3 . This Hecke transitivity statement and the method of prime-to- $p$ monodromy $[6,4.4]$ imply the irreducibility of every non-supersingular distinguished central leaf in $\mathscr{M}_{E}$; see Corollary 9.4. The last irreducibility result in Corollary 9.4 is a crucial ingredient of the proof of the Hecke orbit conjecture for Siegel modular varieties in characteristic $p$; see Remark 9.6. This connection to the Hecke orbit problem was the main motivation of 40 .

The congruity stratification of a Lie stratum of a Hilbert modular variety was first defined in [40, 2.4]; a succinct proof of Theorem 7.1] is in [40, 3.3]. The observation that certain Lie-congruity strata are actually central leaves in Hilbert modular varieties appeared in [12, Section 6], 40, 2.5] and [40, 2.8]. The readers are referred to [9, Chapter 4], which is an expanded version of [40], for more information and proofs.

1.9 .

The length of this article is due partly to a long Section 3 on the definitions of type spaces attached to a totally real number field. Readers who are familiar with basic properties of Hilbert modular varieties are urged to go directly to Section 5 , and consult Section 3 only when necessary.

The rest of this article is organized as follows. In Section 2 we recall the definition of Hilbert modular varieties. In Section 3 we define indexing posets for the Lie stratification of $\mathscr{M}_{E}$ and for the congruity stratification on Lie strata of $\mathscr{M}_{E}$. The stratification of a Hilbert modular variety $\mathscr{M}_{E}$ by Lie type and its basic properties are reviewed in Section 4 . The congruity invariant is defined in Section 5. In Section 6 we explain some basic properties of the congruity stratification of a Lie stratum, including the definition of distinguished Lie congruity strata. The main results on distinguished central leaves, Theorems 7.1 and 8.1. are explained in Sections 7 and 8 respectively. In Section 9 we explain how these two theorems imply the irreducibility of non-supersingular central leaves in Hilbert modular varieties. In Section 10 we discuss some questions on stratifications of $\mathscr{M}_{E}$.

\section{Hilbert modular varieties}

We fix a totally real field $E$, a prime number $p$, and an integer $n \in \mathbb{Z}$ with $n \geq 3$ which is relatively prime to $p$. Let $\mathbb{Z}\left[\zeta_{n}\right]:=\mathbb{Z}[T] /\left(\Phi_{n}(T)\right)$, where $\Phi_{n}(T)$ is the $n$-th cyclotomic polynomial. Let $\mathscr{L}$ be an invertible $\mathcal{O}_{E}$-module, and let $\mathscr{L}_{\mathbb{R}}^{+}$be a notion of positivity for 
$\mathscr{L}$, i.e., a choice of a connected component of

$$
\left(\mathscr{L}_{\mathbb{R}}\right)^{\times}:=\operatorname{Isom}_{\mathcal{O}_{E \otimes_{\mathbb{Q}} \mathbb{R}}}\left(\mathcal{O}_{E \otimes_{\mathbb{Q}} \mathbb{R}}, \mathscr{L} \otimes_{\mathbb{Q}} \mathbb{R}\right)
$$

Let $\widetilde{\mathscr{L}}:=\left(\mathscr{L}, \mathscr{L}_{\mathbb{R}}^{+}\right)$. Let

$$
\bar{\delta} \in\left(\mathscr{L}^{\vee} \otimes_{\mathcal{O}_{E}} \mathcal{D}_{E / \mathbb{Q}}^{-1} / n \mathscr{L}^{\vee} \otimes_{\mathcal{O}_{E}} \mathcal{D}_{E / \mathbb{Q}}^{-1}\right) \otimes_{(\mathbb{Z} / n \mathbb{Z})} \mu_{n}\left(\mathbb{Z}\left[\zeta_{n}, 1 / n\right]\right)
$$

be a generator of the free $\left(\mathcal{O}_{E} / n \mathcal{O}_{E}\right)$-module

$$
\left(\mathscr{L}^{\vee} \otimes_{\mathcal{O}_{E}} \mathcal{D}_{E / \mathbb{Q}}^{-1} / n \mathscr{L}^{\vee} \otimes_{\mathcal{O}_{E}} \mathcal{D}_{E / \mathbb{Q}}^{-1}\right) \otimes_{(\mathbb{Z} / n \mathbb{Z})} \mu_{n}\left(\mathbb{Z}\left[\zeta_{n}, 1 / n\right]\right),
$$

where $\mathscr{L}^{\vee}:=\operatorname{Hom}_{\mathcal{O}_{E}}\left(\mathscr{L}, \mathcal{O}_{E}\right), \mathcal{D}_{E / \mathbb{Q}}^{-1}$ is the inverse different of $E / \mathbb{Q}$, and $\mu_{n}\left(\mathbb{Z}\left[\zeta_{n}, 1 / n\right]\right)$ is the cyclic subgroup of $\mathbb{Z}\left[\zeta_{n}, 1 / n\right]^{\times}$of order $n$ generated by the element $\zeta_{n} \in \mathbb{Z}\left[\zeta_{n}, 1 / n\right]^{\times}$.

In this section we recall the definition of a Hilbert moduli scheme $\mathscr{M}_{E, n, \bar{\delta}}^{\widetilde{\mathscr{L}}}$ over a number $\operatorname{ring} \mathbb{Z}\left[1 / n, \zeta_{n}\right]$ which classifies principally $\widetilde{\mathscr{L}}$-polarized abelian schemes with real multiplication by $\mathcal{O}_{E}$ with $\bar{\delta}$-symplectic level- $n$ structures. Abelian varieties with real multiplication by $\mathcal{O}_{E}$ over $\mathbb{C}$ are uniformized as quotients of $\mathcal{O}_{E} \otimes_{\mathbb{Q}} \mathbb{C}$ by discrete rank-two projective $\mathcal{O}_{E}$-submodules of $\mathcal{O}_{E} \otimes_{\mathbb{Q}} \mathbb{C}$; see Remark 2.2.1(c)(iii). This leads to the uniformization of $\mathscr{M}_{E, n, \bar{\delta}}^{\widetilde{\mathscr{L}}} \times_{\operatorname{Spec}\left(\mathbb{Z}\left[n, \zeta_{n}\right]\right)} \operatorname{Spec}(\mathbb{C})$ and the interpretation of Hilbert modular forms as sections of equivariant line bundles on Hilbert modular varieties. See [35, p. 11] for an informative discussion on the history of Hilbert's modular group and modular functions, including the first papers [2,3] on Hilbert modular forms by Blumenthal, and the correction [26] by Maass of an error in 2,3].

After this section, we will consider only Hilbert modular varieties over $\overline{\mathbb{F}_{p}}$, and the notation $\mathscr{M}_{E, n, \bar{\delta}, \overline{\mathbb{F}_{p}}}^{\widetilde{\mathscr{L}}}$ for a geometric fiber of $\mathscr{M}_{E, n, \bar{\delta}}^{\widetilde{\mathscr{L}}}$ in characteristic $p$ will be shortened to $\mathscr{M}_{E}$.

2.1. Abelian varieties with real multiplication

2.1.1. Let $S$ be a scheme. An abelian scheme with real multiplication by $\mathcal{O}_{E}$ over $S$ is a pair $(A \rightarrow S, \iota)$, where $A \rightarrow S$ is an abelian scheme of relative dimension $[E: \mathbb{Q}]$, and $\iota: \mathcal{O}_{E} \rightarrow \operatorname{End}_{S}(A)$ is a ring homomorphism. Note that $\iota$ is injective because $\operatorname{End}_{S}(A)$ is torsion-free.

2.1.2. Let $(A, \iota)$ be an abelian variety with real multiplication by $\mathcal{O}_{E}$ over a field $K$.

(a) Denote by $\operatorname{Hom}_{\mathcal{O}_{E}}^{\text {sym }}\left(A, A^{t}\right)$ the group of all $K$-homomorphisms $h: A \rightarrow A^{t}$ from $A$ to the dual abelian variety $A^{t}$ such that $h^{t}=h$ under the canonical identification of $A$ with $\left(A^{t}\right)^{t}$. The abelian group $\operatorname{Hom}_{\mathcal{O}_{E}}^{\text {sym }}\left(A, A^{t}\right)$ has a natural structure as a projective $\mathcal{O}_{E}$-module of rank one. 
(b) The $\left(E \otimes_{\mathbb{Q}} \mathbb{R}\right)^{\times}$-torsor

$$
\operatorname{Isom}_{E \otimes_{\mathbb{Q}} \mathbb{R}}\left(E \otimes_{\mathbb{Q}} \mathbb{R}, \operatorname{Hom}_{\mathcal{O}_{E}}^{\mathrm{sym}}\left(A, A^{t}\right) \otimes_{\mathbb{Q}} \mathbb{R}\right)
$$

has a connected component, denoted by $\operatorname{Hom}_{\mathcal{O}_{E}}^{\text {sym }}\left(A, A^{t}\right)_{\mathbb{R}}^{+}$, which is uniquely determined by the property that every element of $\operatorname{Hom}_{\mathcal{O}_{E}}^{\mathrm{sym}}\left(A, A^{t}\right)_{\mathbb{R}}^{+} \cap \operatorname{Hom}_{\mathcal{O}_{E}}^{\text {sym }}\left(A, A^{t}\right)$ is a polarization of the abelian variety $A$.

Remark. The pair $\left(\operatorname{Hom}_{\mathcal{O}_{E}}^{\mathrm{sym}}\left(A, A^{t}\right), \operatorname{Hom}_{\mathcal{O}_{E}}^{\mathrm{sym}}\left(A, A^{t}\right)_{\mathbb{R}}^{+}\right)$attached to an abelian variety $(A, \iota)$ with real multiplication by $\mathcal{O}_{E}$ is an invertible $\mathcal{O}_{E}$-module with a notion of positivity. The set of all isomorphism classes of invertible $\mathcal{O}_{E}$-modules with notions of positivity is the strict class group $\widetilde{\mathrm{Cl}}_{E}$ of $E$. Thus the image of the pair $\left(\operatorname{Hom}_{\mathcal{O}_{E}}^{\mathrm{sym}}\left(A, A^{t}\right), \operatorname{Hom}_{\mathcal{O}_{E}}^{\mathrm{sym}}\left(A, A^{t}\right)_{\mathbb{R}}^{+}\right)$ in $\widetilde{\mathrm{Cl}}_{E}$ is a discrete invariant of $(A, \iota)$.

Definition 2.2. Recall that $\widetilde{\mathscr{L}}=\left(\mathscr{L}, \mathscr{L}_{\mathbb{R}}^{+}\right)$is an invertible $\mathcal{O}_{E}$-module with a notion of positivity. Let $(A \rightarrow S, \iota)$ be an abelian scheme over $S$ with real multiplication by $\mathcal{O}_{E}$.

(1) An $\widetilde{\mathscr{L}}$-polarization of $(A, \iota)$ is an $\mathcal{O}_{E}$-homomorphism $\lambda: \mathscr{L} \rightarrow \operatorname{Hom}_{\mathcal{O}_{E}, S}^{\text {sym }}\left(A, A^{t}\right)$ such that for every point $s \in S$, the map $\lambda_{s}: \mathscr{L} \rightarrow \operatorname{Hom}_{\mathcal{O}_{E}}^{\mathrm{sym}}\left(A_{s}, A_{s}^{t}\right)$ is an isomorphism and sends every element of $\mathscr{L} \cap \mathscr{L}_{\mathbb{R}}^{+}$to an $\mathcal{O}_{E^{-}}$-linear polarization of $A_{s}$.

(2) An $\widetilde{\mathscr{L}}$-polarization $\lambda: \mathscr{L} \rightarrow \operatorname{Hom}_{S}^{\mathrm{sym}}\left(A, A^{t}\right)$ is said to be principal if the natural homomorphism

$$
\mathscr{L} \otimes \mathcal{O}_{E} A \rightarrow A^{t}
$$

is an isomorphism.

Here $\mathscr{L} \otimes_{\mathcal{O}_{E}} A$ is the tensor product of the $\mathcal{O}_{E}$-linear fppf sheaf $A$ over $S$ with the projective $\mathcal{O}_{E}$-module $\mathscr{L}$, which is representable by an abelian scheme over $S$, again denoted by $\mathscr{L} \otimes_{\mathcal{O}_{E}} A$.

Remark 2.2.1. Let $(A, \iota)$ be an abelian variety $A$ with real multiplication by $\mathcal{O}_{E}$ over a field $K$.

(a) The natural map

$$
\alpha_{A}: \operatorname{Hom}_{\mathcal{O}_{E}}^{\mathrm{sym}}\left(A, A^{t}\right) \otimes_{\mathcal{O}_{E}} A \rightarrow A^{t}
$$

is an isogeny. This isogeny $\alpha_{A}$ is an isomorphism if and only if there exists a principal $\left(\operatorname{Hom}_{\mathcal{O}_{E}}^{\mathrm{sym}}\left(A, A^{t}\right), \operatorname{Hom}_{\mathcal{O}_{E}}^{\mathrm{sym}}\left(A, A^{t}\right)_{\mathbb{R}}^{+}\right)$-polarization on $(A, \iota)$.

(b) If the $\mathcal{O}_{E}$-linear abelian variety $(A, \alpha)$ admits an $\mathcal{O}_{E}$-linear principal polarization $\mu: A \rightarrow A^{t}$, then the invariant $\left[\left(\operatorname{Hom}_{\mathcal{O}_{E}}^{\mathrm{sym}}\left(A, A^{t}\right), \operatorname{Hom}_{\mathcal{O}_{E}}^{\mathrm{sym}}\left(A, A^{t}\right)_{\mathbb{R}}^{+}\right)\right]$of $(A, \iota)$ is the zero element of the strict class group $\widetilde{\mathrm{C}} l_{E}$. So the invariant $\left[\left(\operatorname{Hom}_{\mathcal{O}_{E}}^{\mathrm{sym}}\left(A, A^{t}\right)\right.\right.$, $\left.\left.\operatorname{Hom}_{\mathcal{O}_{E}}^{\text {sym }}\left(A, A^{t}\right)_{\mathbb{R}}^{+}\right)\right]$is an obstruction for the existence of an $\mathcal{O}_{E}$-linear principal polarization on $(A, \iota)$. 
(c) Suppose that $K$ is algebraically closed.

(i) If $\operatorname{char}(K)=0$ then the isogeny $\alpha_{A}: \operatorname{Hom}_{\mathcal{O}_{E}}^{\mathrm{sym}}\left(A, A^{t}\right) \otimes_{\mathcal{O}_{E}} A \rightarrow A^{t}$ is an isomorphism.

(ii) If $K=\mathbb{C}$, then $\mathrm{H}_{1}(A(\mathbb{C}), \mathbb{Z})$ is a projective $\mathcal{O}_{E}$-module of rank two, and the invertible $\mathcal{O}_{E}$-module $\operatorname{Hom}_{\mathcal{O}_{E}}^{\text {sym }}\left(A, A^{t}\right)$ is isomorphic to

$$
\operatorname{Hom}_{\mathcal{O}_{E}}\left(\bigwedge_{\mathcal{O}_{E}}^{2} \mathrm{H}_{1}(A(\mathbb{C}), \mathbb{Z}), \mathcal{O}_{E}\right) \otimes \mathcal{O}_{E} \mathcal{D}_{E / \mathbb{Q}}^{-1}
$$

where $\mathcal{D}_{E / \mathbb{Q}}^{-1}$ is the inverse different of $E / \mathbb{Q}$.

It is a fact that for every discrete rank-two $\mathcal{O}_{E}$-submodule $Q$ of $E \otimes_{\mathbb{Q}} \mathbb{C}$, there exists an abelian variety $B$ with real multiplication by $\mathcal{O}_{E}$ over $\mathbb{C}$ such that $B(\mathbb{C})$ is $\mathcal{O}_{E}$-linearly isomorphic to $\left(E \otimes_{\mathbb{Q}} \mathbb{C}\right) / Q$.

(iii) If $\operatorname{char}(K)=p>0$, then $\operatorname{Ker}\left(\alpha_{A}\right)$ is a finite group scheme over $K$ whose order is a power of $p$. Moreover there exists an abelian variety $\left(A_{1}, \iota_{1}\right)$ with real multiplication by $\mathcal{O}_{E}$ over $K$, a principal $\mathcal{O}_{E}$-linear polarization $\mu: A_{1} \rightarrow A_{1}^{t}$, and an $\mathcal{O}_{E}$-linear isogeny $(A, \iota) \rightarrow\left(A_{1}, \iota_{1}\right)$ over $K$.

Remark 2.2.2. There are examples $(A, \iota)$ of abelian varieties with real multiplication by $\mathcal{O}_{E}$ such that the isogeny $\alpha_{A}$ is not an isomorphism, and can be constructed as follows. Suppose that there exists a finite place $v$ of $\mathcal{O}_{E}$ above $p$ with residue field degree $f_{v}>1$. Then there exists an algebraically closed field $K$ of characteristic $p$ and an abelian variety $\left(A_{1}, \iota_{1}\right)$ over $K$ with real multiplication by $\mathcal{O}_{E}$ whose Lie type is not self-dual. It follows from Lemmas 4.2 .2 and 4.2 .3 that the isogeny $\alpha_{A}: \operatorname{Hom}_{\mathcal{O}_{E}}^{\mathrm{sym}}\left(A_{1}, A_{1}^{t}\right) \otimes_{\mathcal{O}_{E}} A_{1} \rightarrow A_{1}^{t}$ is not an isomorphism.

Remark 2.2.3. Let $(A, \lambda, \iota)$ be a principally $\widetilde{\mathscr{L}}$-polarized abelian scheme with real multiplication over a scheme $S$. As explained in Remark 2.2.1(a), there exists an $\mathcal{O}_{E}$-linear principal polarization on $A$ if and only if the image of $\widetilde{\mathscr{L}}$ in the strict class group $\widetilde{\mathrm{C}} l_{E}$ is 0 . If the element $[\widetilde{\mathscr{L}}] \in \widetilde{\mathrm{Cl}} l_{E}$ of $\widetilde{\mathscr{L}}$ is nonzero, then there is no $\mathcal{O}_{E}$-linear principal polarization $A \rightarrow A^{t}$, but there exist (many) separable $\mathcal{O}_{E}$-linear polarizations on $A$ : by weak approximation for $\widetilde{\mathscr{L}}$, every element of $\mathscr{L} \cap \mathscr{L}_{\mathbb{R}}^{+}$whose image in $\mathscr{L} / p \mathscr{L}$ is a generator of the cyclic $\left(\mathcal{O}_{E} / p \mathcal{O}_{E}\right)$-module $\mathscr{L} / p \mathscr{L}$ corresponds to an $\mathcal{O}_{E}$-linear separable polarization of $(A, \iota)$.

Definition 2.3. (a) Recall that $\bar{\delta}$ is a free generator of the $\left(\mathcal{O}_{E} / n \mathcal{O}_{E}\right)$-module

$$
\left(\mathscr{L}^{\vee} \otimes \mathcal{D}_{E / \mathbb{Q}}^{-1} / n \mathscr{L}^{\vee} \otimes \mathcal{D}_{E / \mathbb{Q}}^{-1}\right) \otimes_{(\mathbb{Z} / n \mathbb{Z})} \mu_{n}\left(\mathbb{Z}\left[\zeta_{n}, 1 / n\right]\right)
$$


For each element $l \in \mathscr{L}$, denote by

$$
\langle\cdot, \cdot\rangle_{n, \bar{\delta}, l}:\left(\mathcal{O}_{E} / n \mathcal{O}_{E}\right)_{S}^{\oplus 2} \rightarrow \mu_{n, S}
$$

the alternating pairing on the free module $\left(\mathcal{O}_{E} / n \mathcal{O}_{E}\right)_{S}^{\oplus 2}$ over the sheaf of rings $\left(\mathcal{O}_{E} / n \mathcal{O}_{E}\right)_{S}$ on $S_{\text {et }}$ attached to the free $\left(\mathcal{O}_{E} / n \mathcal{O}_{E}\right)$-module $\left(\mathcal{O}_{E} / n \mathcal{O}_{E}\right)^{\oplus 2}$ with generators $\boldsymbol{e}_{1}, \boldsymbol{e}_{2}$, which is equal to the composition of the canonical $\left(\mathcal{O}_{E} / n \mathcal{O}_{E}\right)_{S}$-linear alternating pairing

$$
\left(\mathcal{O}_{E} / n \mathcal{O}_{E}\right)_{S}^{\oplus 2} \rightarrow \bigwedge_{\left(\mathcal{O}_{E} / n \mathcal{O}_{E}\right)}^{2}\left(\mathcal{O}_{E} / n \mathcal{O}_{E}\right)_{S}^{\oplus 2}
$$

with the homomorphism of group schemes

$$
\bigwedge_{\left(\mathcal{O}_{E} / n \mathcal{O}_{E}\right)}^{2}\left(\mathcal{O}_{E} / n \mathcal{O}_{E}\right)_{S}^{\oplus 2} \rightarrow \mu_{n, S}, \quad a \cdot \boldsymbol{e}_{1} \wedge \boldsymbol{e}_{2} \mapsto\left(\operatorname{Tr}_{E / \mathbb{Q}} \otimes 1\right)(l \cdot \bar{\delta} \cdot a)
$$

for all local sections $a$ of $\left(\mathcal{O}_{E} / n \mathcal{O}_{E}\right)_{S}$, where

$$
\operatorname{Tr}_{E / \mathbb{Q}} \otimes 1:\left(\mathcal{D}_{E / \mathbb{Q}}^{-1} / n \mathcal{D}_{E / \mathbb{Q}}^{-1}\right) \otimes_{(\mathbb{Z} / n \mathbb{Z})} \mu_{n, S} \rightarrow \mu_{n, S}
$$

is the homomorphism of group schemes induced by the $(E / \mathbb{Q})$-trace.

(b) Let $S$ be a scheme over $\mathbb{Z}\left[1 / n, \zeta_{n}\right]$. Let $(A \rightarrow S, \iota)$ be an abelian scheme over $S$ with real multiplication by $\mathcal{O}_{E}$, and let $\lambda$ be a principal $\widetilde{\mathscr{L}}$-polarization of $(A, \iota)$.

A $\bar{\delta}$-symplectic level-n structure on $(A, \lambda, \iota)$ is an $\mathcal{O}_{E}$-linear isomorphism

$$
\left(\mathcal{O}_{E} / n \mathcal{O}_{E}\right)_{S}^{2} \stackrel{\eta}{\simeq} A[n]
$$

such that for every $l \in \mathscr{L}$, the diagram

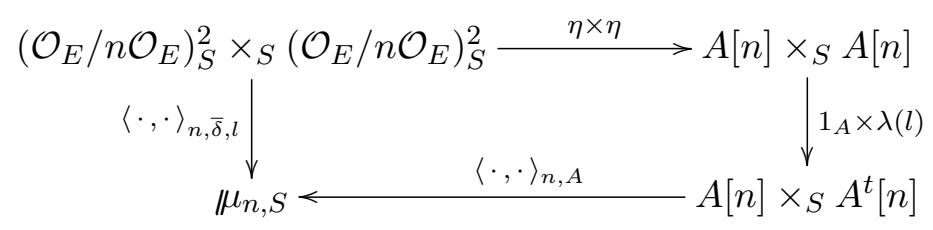

commutes, where

$$
\langle\cdot, \cdot\rangle_{n, A}: A[n] \times_{S} A^{t}[n] \rightarrow \mu_{n, S}
$$

is the Weil pairing for $A[n]$.

Definition 2.4. (a) Denote by

$$
\mathscr{M}_{E, n, \bar{\delta}}^{\widetilde{\mathscr{L}}}:\left(\mathbb{Z}\left[1 / n, \zeta_{n}\right] \text {-Schemes }\right) \rightarrow(\text { Sets })
$$

the functor from the category of $\mathbb{Z}\left[1 / n, \zeta_{n}\right]$-schemes to the category of sets as follows. For every $\mathbb{Z}\left[1 / n, \zeta_{n}\right]$-scheme $S, \mathscr{M}_{E, n, \bar{\delta}}^{\widetilde{\mathscr{L}}}(S)$ is the set of all isomorphism classes of quadruples 
$(A \rightarrow S, \lambda, \iota, \eta)$, where $(A \rightarrow S, \iota)$ is an abelian scheme with real multiplication by $\mathcal{O}_{E}$ over $S, \lambda$ is a principal $\widetilde{\mathscr{L}}$-polarization of $(A, \iota)$, and $\eta$ is a $\bar{\delta}$-symplectic level- $n$ structure.

(b) Pick and fix a ring homomorphism from $\mathbb{Z}\left[1 / n, \zeta_{n}\right]$ an algebraic closure of $\overline{\mathbb{F}_{p}}$. Denote by $\mathscr{M}_{E, n, \bar{\delta}, \overline{\mathbb{F}_{p}}}^{\widetilde{\mathscr{L}}}$ the restriction of the moduli functor $\mathscr{M}_{E, n, \bar{\delta}}^{\widetilde{\mathscr{L}}}$ to the category of $\overline{\mathbb{F}_{p^{-}}}$ schemes.

Proposition 2.5. (a) The functor $\mathscr{M}_{E, n, \bar{\delta}}^{\widetilde{\mathscr{L}}}$ is representable by a scheme of finite type over $\mathbb{Z}\left[1 / n, \zeta_{n}\right]$, again denoted by $\mathscr{M}_{E, n, \bar{\delta}}^{\widetilde{\mathscr{L}}}$. The structural morphism $\mathscr{M}_{E, n, \bar{\delta}}^{\widetilde{\mathscr{L}}} \rightarrow$ $\operatorname{Spec}\left(\mathbb{Z}\left[1 / n, \zeta_{n}\right]\right)$ is faithfully flat, normal and is a relative complete intersection.

(b) The geometric fiber $\mathscr{M}_{E, n, \bar{\delta}, \overline{\mathbb{F}_{p}}}^{\widetilde{\mathscr{L}}}$ is normal and irreducible. It is smooth over $\overline{\mathbb{F}_{p}}$ if and only if $E / \mathbb{Q}$ is unramified above $p$.

(c) Suppose that $E / \mathbb{Q}$ is ramified above $p$. Then the smooth locus $\mathscr{M}_{E, n, \bar{\delta}, \overline{\mathbb{F}_{p}}}^{\widetilde{\mathscr{L}}}$ of $\mathscr{M}_{E, n, \bar{\delta}, \overline{\mathbb{F}_{p}}}^{\widetilde{L}}$ is a dense open subscheme of $\mathscr{M}_{E, n, \overline{\mathscr{L}}, \overline{\mathbb{F}_{p}}}^{\widetilde{L}}$. The singular locus

$$
V_{\text {sing }}:=\mathscr{M}_{E, n, \bar{\delta}, \overline{\mathbb{F}_{p}}}^{\widetilde{\mathscr{L}}} \backslash \mathscr{M}_{E, n, \bar{\delta}, \overline{\mathbb{F}_{p}}}^{\widetilde{\mathscr{L}} \mathrm{sm}}
$$

with the reduced structure is a local complete intersection, and every irreducible component of $V_{\text {sing }}$ is of codimension 2 in $\mathscr{M}_{E, n, \bar{\delta}}^{\widetilde{L}}$.

Proposition 2.5 is a consequence of 33 , Theorem 5.1] and 13, Theorem 3.3]. Here we explain the irreducibility statement (b). We have an arithmetic toroidal compactification $\mathscr{M}_{E, n, \bar{\delta}}^{\widetilde{\mathscr{L}}, \Sigma}$ of $\mathscr{M}_{E, n, \bar{\delta}}^{\widetilde{\mathscr{L}}}$ over $\mathbb{Z}\left[1 / n, \zeta_{n}\right]$ which is again a complete intersection over $\mathbb{Z}\left[1 / n, \zeta_{n}\right]$. It follows that the geometric fiber $\mathscr{M}_{E, n, \bar{\delta}, \overline{\mathbb{F}_{p}}}^{\widetilde{\mathscr{L}}, \Sigma}$ is normal. Since the geometric generic fiber of $\mathscr{M}_{E, n, \bar{\delta}}^{\widetilde{\mathscr{L}}}$ is geometrically irreducible by complex uniformization, so is the geometric fiber in characteristic $p$.

Remark 2.5.1. As a special case of Remark 2.2.3, the universal abelian scheme $\boldsymbol{A} \rightarrow$ $\mathscr{M}_{E, n, \bar{\delta}, \overline{\mathbb{F}_{p}}}^{\widetilde{\mathscr{L}}}$ with real multiplication by $\mathcal{O}_{E}$ has many separable $\mathcal{O}_{E}$-linear polarizations, and it admits an $\mathcal{O}_{E}$-linear principal polarization if and only if the element $[\widetilde{\mathscr{L}}]$ in the strict class group of $E$ is zero. If $[\widetilde{\mathscr{L}}]$ is non-zero, one can view the $\mathscr{M}_{E, n, \overline{\mathscr{\delta}}, \overline{\mathbb{F}_{p}}}^{\widetilde{\mathscr{L}}}$ as an analog of an irreducible component of $\mathscr{A}_{g, d, n}$ with $\operatorname{gcd}(d, p)=1$, where $\mathscr{A}_{g, d, n}$ is the moduli space over $\overline{\mathbb{F}_{p}}$ of $g$-dimensional polarized abelian varieties $(A, \lambda, \eta)$ with $\operatorname{deg} \lambda=d^{2}$ and level- $n$ structure.

2.5.2. In the rest of this article we will consider only Hilbert modular varieties $\mathscr{M}_{E, n, \bar{\delta}, \overline{\mathbb{F}_{p}}}^{\widetilde{\mathscr{L}}}$ over $\overline{\mathbb{F}_{p}}$. Often we will also suppress the parameters $\widetilde{\mathscr{L}}, n$ and $\bar{\delta}$ from the notation, and shorten $\mathscr{M}_{E, n, \bar{\delta}, \overline{\mathbb{F}}_{p}}^{\widetilde{\mathscr{L}}}$ to $\mathscr{M}_{E}$, if there is no risk of confusion. 


\section{Type spaces}

This section contains the definitions of two finite posets $\mathcal{T}_{E}^{\mathrm{sd}}, \boldsymbol{\tau}_{E}$ associated to the totally real number field $E$. They are natural indexing sets for the Lie stratification of the Hilbert modular variety $\mathscr{M}_{E}$, and for the congruity stratification of Lie strata of $\mathscr{M}_{E}$. The partial ordering of these posets are compatible with the incidence relation between strata in the respective stratifications.

We recommend readers to go directly to Definition 3.2.1 and skip the rest of this section, and return to this section only when necessary.

\subsection{Product decomposition of the $\operatorname{ring} \mathcal{O}_{E} \otimes_{\mathbb{Z}} \Lambda\left(\overline{\mathbb{F}_{p}}\right)$}

3.1.1. Notations related to the totally real field $E$ and its $p$-adic completion $E \otimes_{\mathbb{Q}} \mathbb{Q}_{p}$.

- Denote by $\Sigma_{E, p}$ the set of places of $E$ above $p$.

- Let $v \in \Sigma_{E, p}$ be a place of $E$ above $p$ and let $E_{v}$ the $v$-adic completion of $E$ at $v$.

- Let $\mathcal{O}_{v}=\mathcal{O}_{E_{v}}$ be the ring of integers in $E_{v}$, let $\wp_{v}$ be the maximal ideal of $\mathcal{O}_{E_{v}}$, and let $\kappa_{v}:=\mathcal{O}_{E_{v}} / \wp_{v}$, the residue field of $\mathcal{O}_{E_{v}}$.

- Let $g_{v}:=\left[E_{v}: \mathbb{Q}_{p}\right], f_{v}:=\left[\kappa_{v}: \overline{\mathbb{F}_{p}}\right]$, and let $e_{v}=e\left(E_{v} / \mathbb{Q}_{p}\right)$ be the absolute ramification index of $E_{v}$.

- Denote by $\pi_{v}$ a generator of the maximal ideal $\wp_{v}$ of $\mathcal{O}_{v}$.

- Let $E_{v}^{\text {ur }}$ be the maximal subfield of $E_{v}$ unramified over $\mathbb{Q}_{p}$, and let $\mathcal{O}_{E_{v}^{\text {ur }}}$ be the ring of integers of $E_{v}^{u r}$.

- Let $\operatorname{Fr}_{\kappa_{v}}$ be the arithmetic Frobenius map $\left(y \mapsto y^{p}\right)$ on $\kappa_{v}$. We will identify the Galois group $\operatorname{Gal}\left(\kappa_{v} / \mathbb{F}_{p}\right)$ with $\mathbb{Z} / f_{v} \mathbb{Z}$, by the isomorphism which sends the element $\operatorname{Fr}_{\kappa_{v}}$ in $\operatorname{Gal}\left(\kappa_{v} / \mathbb{F}_{p}\right)$ to the element $1+f_{v} \mathbb{Z}$ in $\mathbb{Z} / f_{v} \mathbb{Z}$.

- Let $f_{E}:=\operatorname{lcm}\left\{f_{v} \mid v \in \Sigma_{E, p}\right\}$, the least common multiple of all $f_{v}$ 's.

- Let $\kappa$ be perfect field containing $\kappa_{E}$, where $\kappa_{E}$ denotes a finite field with $p^{f_{E}}$ elements.

- Denote by $\Lambda(\kappa)$ the ring of all $p$-adic Witt vectors with entries in $\kappa$.

- Let $\sigma$ be the continuous ring automorphism of $\Lambda(\kappa)$, which sends every infinite Witt vector $\left(a_{0}, a_{1}, \ldots, a_{n}, \ldots\right)$ in $\Lambda(\kappa)$ to $\left(a_{0}^{p}, a_{1}^{p}, \ldots, a_{n}^{p}, \ldots\right)$.

- For every $v \in \Sigma_{E, p}$, define a torsor $I_{v}=I_{v, \kappa}$ for the cyclic group $\operatorname{Gal}\left(\kappa_{v} / \mathbb{F}_{p}\right)=$ $\mathbb{Z} / f_{v} \mathbb{Z}$ by

$$
I_{v}=I_{v, \kappa}:=\operatorname{Hom}_{\text {ring,cont }}\left(\mathcal{O}_{E_{v}^{\mathrm{ur}}}, \Lambda(\kappa)\right) \stackrel{\sim}{\rightarrow} \operatorname{Hom}_{\text {ring }}\left(\kappa_{v}, \kappa\right),
$$

so that for every element $i \in I_{v, \kappa}$, the element $1 \bmod f_{v} \in \mathbb{Z} / f_{v} \mathbb{Z}$ sends $i$ to the element $\sigma \circ i \in I_{v, \kappa}$. 
3.1.2. We have direct product decompositions of rings

$$
\mathcal{O}_{E} \otimes_{\mathbb{Z}} \mathbb{Z}_{p}=\prod_{v \in \Sigma_{E, p}} \mathcal{O}_{E_{v}} \quad \text { and } \quad \mathcal{O}_{E} \otimes_{\mathbb{Z}} \mathbb{F}_{p}=\prod_{v \in \Sigma_{E, p}} \mathcal{O}_{E_{v}} / \wp_{v}^{e_{v}}
$$

For every perfect field $\kappa$ containing $\kappa_{E}$, we have direct sum decompositions of rings

$$
\mathcal{O}_{E_{v}} \otimes_{\mathbb{Z}_{p}} \Lambda(\kappa)=\prod_{i \in I_{v, \kappa}} \mathcal{O}_{E_{v}} \otimes_{\left(\mathcal{O}_{E_{v}}^{\mathrm{ur}}, i\right)} \Lambda(\kappa) \quad \text { and } \quad \mathcal{O}_{E_{v}} \otimes_{\mathbb{Z}_{p}} \kappa=\prod_{i \in I_{v, \kappa}} \mathcal{O}_{E_{v}} \otimes_{\left(\mathcal{O}_{E_{v}}^{\mathrm{ur}, i)}\right.} \kappa .
$$

So we get a product decomposition

$$
\mathcal{O}_{E} \otimes_{\mathbb{Z}} \Lambda(\kappa)=\prod_{v \in \Sigma_{E, p}} \prod_{i \in I_{v, \kappa}} \mathcal{O}_{E_{v}} \otimes_{\left(\mathcal{O}_{E_{v}}^{\mathrm{ur}}, i\right)} \Lambda(\kappa)=\prod_{v \in \Sigma_{E, p}} \prod_{i \in I_{v, \kappa}} \Lambda(\kappa)_{v}^{(i)},
$$

where $\Lambda(\kappa)_{v}^{(i)}$ denotes the complete discrete valuation ring

$$
\Lambda(\kappa)_{v}^{(i)}:=\mathcal{O}_{E_{v}} \otimes_{\left(\mathcal{O}_{\left.E_{v}^{\mathrm{ur}}, i\right)}\right.} \Lambda(\kappa)
$$

with residue field $\kappa$ and absolute ramification index $e_{v}$. The maximal ideal of $\Lambda^{(i)}=\Lambda(\kappa)^{(i)}$ is generated by the element $\pi_{v} \in \mathcal{O}_{E_{v}}$. From the product decomposition (3.1) of the ring $\mathcal{O}_{E} \otimes_{\mathbb{Z}} \Lambda(\kappa)$, we see that every finitely generated module over $\mathcal{O}_{E} \otimes_{\mathbb{Z}} \Lambda(\kappa)$ is a direct sum, over all pairs $(v, i)$, of direct sums of cyclic modules of the discrete valuation rings $\Lambda_{v}^{(i)}$.

Similarly, for every field $K$ containing $\kappa_{E}$, not necessarily perfect, we have a product decomposition

$$
\mathcal{O}_{E} \otimes_{\mathbb{Z}} K=\prod_{v \in \Sigma_{E, p}} \prod_{i \in I_{v, K}} \mathcal{O}_{E_{v}} \otimes_{\left(\mathcal{O}_{E_{v}}^{\mathrm{ur}}, i\right)} K=\prod_{v \in \Sigma_{E, p}} \prod_{i \in I_{v, K}}\left(\kappa_{v}[T] /\left(T^{e_{v}}\right)\right) \otimes_{\left(\kappa_{v}, i\right)} K,
$$

where

$$
I_{v, K}=I_{v, \kappa_{E}}:=\operatorname{Hom}_{\text {ring }}\left(\kappa_{v}, \kappa_{E}\right)=\operatorname{Hom}_{\text {ring }}\left(\kappa_{v}, K\right)
$$

for every $v \in \Sigma_{E, p}$, and $\kappa_{v}[T]$ is the polynomial ring over $\kappa_{v}$ in the variable $T$. It follows that every finitely generated module over $\mathcal{O}_{E} \otimes_{\mathbb{Z}} K$ is isomorphic to a direct sum, over all pairs $(v, i)$, of direct sum of cyclic modules of the ring

$$
\left(\kappa_{v}[T] /\left(T^{e_{v}}\right)\right) \otimes_{\left(\kappa_{v}, i\right)} K \cong K[T] / T^{e_{v}} K[T]
$$

\subsection{Type spaces of finite $\left(\mathcal{O}_{E} \otimes_{\mathbb{Z}} K\right)$-modules}

In this subsection, $K$ is a field containing $\kappa_{E}$. The type spaces considered in this article are isomorphisms classes of modules over $\mathcal{O}_{E} \otimes K$ which can be generated by two elements, together with natural partial ordering compatible with specialization.

Definition 3.2.1. Let $K \supseteq \mathbb{F}_{p}$ be a field which contains a finite subfield $\kappa_{E}$ with $p^{f_{E}}$ elements. 
(i) Denote by $\mathcal{T}_{E, K}$ to be the set of all isomorphism classes of modules over $\mathcal{O}_{E} \otimes K$ which can be generated by two elements.

We will identify $\mathcal{T}_{E, K}$ with the set consisting of all collections of sequences of pairs of the form

$$
\begin{aligned}
& \quad \underline{e}=\left(\underline{e}_{v}\right)_{v \in \Sigma_{E, p}}=\left(\left(e_{v, 1}^{(i)}, e_{v, 2}^{(i)}\right)_{i \in I_{v, K}}\right)_{v \in \Sigma_{E, p}} \\
& \text { s.t. } \quad e_{v, 1}^{(i)}, e_{v, 2}^{(i)} \in\left\{0,1, \ldots, e_{v}\right\}, \quad e_{v, 1}^{(i)} \leq e_{v, 2}^{(i)}
\end{aligned}
$$

for all $v \in \Sigma_{E, p}$ and all $i \in I_{v, K}$. An element $\underline{e}$ as above corresponds to the isomorphism class of the $\left(\mathcal{O}_{E} \otimes_{\mathbb{Z}} K\right)$-module

$$
\begin{aligned}
\bigoplus_{v \in \Sigma_{E, p}} \bigoplus_{i \in I_{v, K}}\left(\mathcal{O}_{E_{v}} \otimes_{\left(\mathcal{O}_{\left.E_{v}^{\mathrm{ur}}, i\right)} K\right.} K / \pi_{v}^{e_{v, 1}^{(i)}} \mathcal{O}_{E_{v}} \otimes_{\left(\mathcal{O}_{\left.E_{v}, i\right)}, i\right)} K\right) \\
\oplus\left(\mathcal{O}_{E_{v}} \otimes_{\left(\mathcal{O}_{E_{v}}^{\mathrm{ur}}, i\right)} K / \pi_{v}^{e_{v, 2}^{(i)}} \mathcal{O}_{E_{v}} \otimes_{\left(\mathcal{O}_{\left.E_{v}^{\mathrm{ur}}, i\right)} K\right) .}\right.
\end{aligned}
$$

(ii) Let $\boldsymbol{K}\left(\mathcal{O}_{E} \otimes_{\mathbb{Z}} K\right)$ denote the Grothendieck group of finitely generated $\left(\mathcal{O}_{E} \otimes_{\mathbb{Z}} K\right)$ modules. Denote by $\mathcal{T}_{E, K}^{\text {sd }} \subset \mathcal{T}_{E, K}$ the subset consisting of all isomorphism classes of modules over $\mathcal{O}_{E} \otimes_{\mathbb{Z}} K$ whose image in $\boldsymbol{K}\left(\mathcal{O}_{E} \otimes K\right)$ is equal to the image of $\mathcal{O}_{E} \otimes K$ in $\boldsymbol{K}\left(\mathcal{O}_{E} \otimes_{\mathbb{Z}} K\right)$.

Following the explicit form 3.3 of $\mathcal{T}_{E, K}$, we will identify the type space $\mathcal{T}_{E, K}^{\mathrm{sd}}$ with the set of all collections of sequences of pairs

$$
\left(\left(e_{v, 1}^{(i)}, e_{v, 2}^{(i)}\right)_{i \in I_{v, K}}\right)_{v \in \Sigma_{E, p}} \quad \text { s.t. } \quad e_{v, 1}^{(i)}, e_{v, 2}^{(i)} \in \mathbb{N}, e_{v, 1}^{(i)} \leq e_{v, 2}^{(i)}, e_{v, 1}^{(i)}+e_{v, 2}^{(i)}=e_{v}
$$

for all $v \in \Sigma_{E, p}$ and all $i \in I_{v, K}$. Here $\mathbb{N}$ denotes the set of all non-negative integers.

(iii) Denote by $\boldsymbol{\tau}_{E, K}$ the set of all collections of sequences

$$
\underline{c}=\left(\underline{c}_{v}\right)_{v \in \Sigma_{E, p}}=\left(\left(c_{v}^{(i)}\right)_{i \in I_{v, K}}\right)_{v \in \Sigma_{E, p}} \quad \text { s.t. } \quad 0 \leq c_{v}^{(i)} \leq e_{v}
$$

for all $v \in \Sigma_{E, p}$ and all $i \in I_{v, K}$. We will identify $\boldsymbol{\tau}_{E, K}$ with the set of all isomorphism classes of cyclic $\left(\mathcal{O}_{E} \otimes_{\mathbb{Z}} K\right)$-modules.

Remark 3.2.2. (a) For any two fields $K, K^{\prime}$ containing $\kappa_{E}$, every ring homomorphism $h: K \rightarrow K^{\prime}$ induces isomorphisms

$$
I_{v, K} \stackrel{\sim}{\rightarrow} I_{v, K^{\prime}}, \quad \mathcal{T}_{E, K} \stackrel{\sim}{\rightarrow} \mathcal{T}_{E, K^{\prime}}, \quad \mathcal{T}_{E, K}^{\mathrm{sd}} \stackrel{\sim}{\rightarrow} \mathcal{T}_{E, K^{\prime}}^{\mathrm{sd}}, \quad \boldsymbol{\tau}_{E, K} \stackrel{\sim}{\rightarrow} \boldsymbol{\tau}_{E, K^{\prime}}
$$

Often we will suppress the subscript " $K$ " and shorten the notations $I_{v, K}, \mathcal{T}_{E, K}, \mathcal{T}_{E, K}^{\mathrm{sd}}$, $\tau_{E, K}$ to $I_{v}, \mathcal{T}_{E}, \mathcal{T}_{E}^{\mathrm{sd}}, \tau_{E}$ respectively, when there is no risk of confusion. 
(b) The type space $\mathcal{T}_{E}$ is a direct product of spaces $\mathcal{T}_{E_{v}}$, where $v$ runs through all $v \in \Sigma_{E, p}$. The same holds for $\mathcal{T}_{E}^{\text {sd }}$ and $\boldsymbol{\tau}_{E}$. Explicitly, $\mathcal{T}_{E, v}$ consists of all sequences of pairs

$$
\left(e_{v, 1}^{(i)}, e_{v, 2}^{(i)}\right)_{i \in I_{v}}, \quad e_{v, 1}^{(i)}, e_{v, 2}^{(i)} \in\left\{0,1, \ldots, e_{v}\right\}, \quad e_{v, 1}^{(i)} \leq e_{v, 2}^{(i)}, \quad \forall i \in I_{v},
$$

and similarly for $\mathcal{T}_{E}^{\text {sd }}$ and $\boldsymbol{\tau}_{E}$.

(c) Elements of $\boldsymbol{T}_{E, K}^{\mathrm{sd}}$ and $\mathcal{T}_{E, K}^{\mathrm{sd}}$ will be called self-dual types. The Lie type of any principally $\widetilde{\mathscr{L}}$-polarized abelian variety with real multiplication by $\mathcal{O}_{E}$ over $K$ is self-dual. Similarly the Lie type of any self-dual rank-two $\mathcal{O}_{E}$-linear $p$-divisible group over $K$, is self-dual. See Lemmas 4.2 .2 and 4.2 .3 .

Definition 3.3. We define partial orderings on the type spaces $\mathcal{T}_{E}, \mathcal{T}_{E}^{s d}$ and $\boldsymbol{\tau}_{E}$ as follows.

(i) Let $\underline{e}_{1}, \underline{e}_{2}$ be two elements of $\mathcal{T}_{E}, \underline{e}_{j}=\left(\left(e_{j ; v, 1}^{(i)}, e_{j ; v, 2}^{(i)}\right)_{i \in I_{v, k}}\right)_{v \in \Sigma_{E, p}}$ for $j=1,2$. Define $\underline{e_{2}} \preceq \underline{e_{1}}$ if

$$
e_{1 ; v, 1}^{(i)} \leq e_{2 ; v, 1}^{(i)} \quad \text { and } \quad e_{1 ; v, 1}^{(i)}+e_{1 ; v, 2}^{(i)} \leq e_{2 ; v, 1}^{(i)}+e_{2 ; v, 2}^{(i)}, \quad \forall v \in \Sigma_{E, p}, \forall i \in I_{v}
$$

for all $v \in \Sigma_{E, p}$ and all $i \in I_{v}$.

(ii) The restriction of the partial ordering $\preceq$ on $\mathcal{T}_{E}$ induces a partial ordering on $\mathcal{T}_{E}^{\text {sd }}$. Explicitly, if $\underline{e}_{1}, \underline{e}_{2}$ are two elements of $\mathcal{T}_{E}^{\mathrm{sd}}$ with $\underline{e}_{j}=\left(\left(e_{j ; v, 1}^{(i)}, e_{j ; v, 2}^{(i)}\right)_{i \in I_{v, k}}\right)_{v \in \Sigma_{E, p}}$ for $j=1,2$, then

$$
\underline{e_{2}} \preceq \underline{e_{1}} \quad \Longleftrightarrow \quad e_{1 ; v, 1}^{(i)} \leq e_{2 ; v, 1}^{(i)}, \quad \forall v, i .
$$

Note that the last condition is equivalent to $e_{2 ; v, 2}^{(i)} \leq e_{1 ; v, 2}^{(i)}$ for all $v \in \Sigma_{E, p}$ and all $i \in I_{v}$.

(iii) For any two elements $\underline{c}_{j}=\left(c_{j ; v}^{(i)}\right)_{v \in \Sigma_{E, p}, i \in I_{v}}$ in $\boldsymbol{\tau}_{E}, j=1,2$,

$$
\underline{c}_{2} \preceq \underline{c}_{1} \quad \Longleftrightarrow \quad c_{1 ; v}^{(i)} \leq c_{2 ; v}^{(i)}, \quad \forall v \in \Sigma_{E, p}, \forall i \in I_{v} .
$$

Remark 3.3.1. (a) The poset $\mathcal{T}_{E}^{\mathrm{sd}}$ is ranked, in the sense that any two maximal chain between two comparable elements have the same length.

(b) The poset $\mathcal{T}_{E}^{\mathrm{sd}}$ has a unique maximal element $\underline{e}_{\max }$ and a unique minimal element $\underline{e}_{\min }$. The $(v, i)$-component $\left(e_{\max , v, 1}^{(i)}, e_{\max , v, 2}^{(i)}\right)$ of $\underline{e}_{\max }$ is $\left(0, e_{v}\right)$ for all $v \in \Sigma_{E, p}$ and all $i \in I_{v}$. The $(v, i)$-component $\left(e_{\min , v, 1}^{(i)}, e_{\min , v, 2}^{(i)}\right)$ of $\underline{e}_{\min }$ is $\left(\left\lfloor e_{v} / 2\right\rfloor,\left\lceil e_{v} / 2\right\rceil\right)$ for $v \in \Sigma_{E, p}$ and all $i \in I_{v}$. Here $\left\lfloor e_{v} / 2\right\rfloor$ is rounding $e_{v} / 2$ down to the nearest integer, while $\left\lceil e_{v} / 2\right\rceil$ is rounding $e_{v} / 2$ up to the nearest integer.

3.3.2. Let $S$ be a scheme over a field containing $\kappa_{E}$. Let $M$ be a coherent $\mathcal{O}_{S}$ module, endowed with an action by $\mathcal{O}_{E}$, i.e., a ring homomorphism $\mathcal{O}_{E} \rightarrow \operatorname{End}_{S}(M)$. For every 
point $s \in S$, the product decomposition 3.2 with $K$ replaced by the residue field $\kappa(s)$ at $s$ induces a decomposition of the fiber $M_{s}$ as a module over $\mathcal{O}_{E} \otimes_{\mathbb{Z}} \kappa(s)$. Denote by $\underline{e}_{M, s}$ the element in the type space $\mathcal{T}_{E, \kappa(s)} \stackrel{\sim}{\leftarrow} \mathcal{T}_{E, K}$ determined by the $\left(\mathcal{O}_{E} \otimes_{\mathbb{Z}} \kappa(s)\right)$-module $M_{s}$.

Lemma 3.3.3. Let $M$ be a coherent $\mathcal{O}_{S}$-module as in Subsubsection 3.3.2. Let $s$, $s^{\prime}$ be points of $S$. If $s$ is a specialization of $s^{\prime}$, then $\underline{e}_{M, s} \preceq \underline{e}_{M, s^{\prime}}$

The proof of Lemma 3.3 .3 is left as an exercise.

Definition 3.3.4. Define a function strdim: $\mathcal{T}_{E, k}^{\mathrm{sd}} \rightarrow \mathbb{N}$ by

$$
\operatorname{strdim}(\underline{e})=\sum_{v \in \Sigma_{E, p}} \sum_{i \in I_{v}}\left(e_{v, 2}^{(i)}-e_{v, 1}^{(i)}\right)
$$

if $\underline{e}=\left(\left(\left(e_{v, 1}^{(i)}, e_{v, 2}^{(i)}\right)\right)_{i \in I_{v}}\right)_{v \in \Sigma_{E, p}}$.

Definition 3.4 (Local version of Definition 3.2.1). Let $v$ be a place of $E$ above $p$ and let $K$ be a field which contains a subfield with $p^{f_{v}}$ elements. Let $I_{v, K}=\operatorname{Hom}_{\text {ring }}\left(\kappa_{v}, K\right)$, regarded as a torsor over $\operatorname{Gal}\left(\kappa_{v} / \mathbb{F}_{p}\right) \cong \mathbb{Z} / f_{v} \mathbb{Z}$. The ring $\mathcal{O}_{E_{v}} \otimes_{\mathbb{Z}_{p}} K$ admits a natural product decomposition

$$
\mathcal{O}_{E_{v}} \otimes_{\mathbb{Z}_{p}} K=\prod_{i \in I_{v, K}}\left(\mathcal{O}_{E_{v}} \otimes_{\mathcal{O}_{E_{v}}^{\mathrm{ur}}, i} K\right)
$$

(i) Denote by $\mathcal{T}_{E_{v}, K}$ the set of all isomorphism classes of modules over $\mathcal{O}_{E_{v}} \otimes_{\mathbb{Z}_{p}} K$ which can be generated by two elements. We identify $\mathcal{T}_{E_{v}, K}$ with the set consisting of all sequences of pairs

$$
\underline{e}_{v}=\left(\left(b_{1}^{(i)}, b_{2}^{(i)}\right)\right)_{i \in I_{v, K}}, \quad 0 \leq b_{1}^{(i)} \leq b_{2}^{(i)} \leq e_{v}, \quad \forall i \in I_{v, K} .
$$

A sequence of pairs as above corresponds to the isomorphism class of the $\left(\mathcal{O}_{E_{v}} \otimes_{\mathbb{Z}_{p}}\right.$ $K$ )-module

$$
\bigoplus_{i \in I_{v, K}}\left(\mathcal{O}_{E_{v}} \otimes_{\mathcal{O}_{E_{v}}^{\mathrm{ur}, i}} K\right) /\left(\pi_{v}^{b_{1}^{(i)}} \mathcal{O}_{E_{v}} \otimes_{\mathcal{O}_{E_{v}^{u r}, i}} K\right) \oplus\left(\mathcal{O}_{E_{v}} \otimes_{\mathcal{O}_{E_{v}}^{\mathrm{ur}, i}} K\right) /\left(\pi_{v}^{b_{2}^{(i)}} \mathcal{O}_{E_{v}} \otimes_{\mathcal{O}_{E_{v}}^{\mathrm{ur}, i}} K\right)
$$

(ii) Denote by $\mathcal{T}_{E_{v}, K}^{\mathrm{sd}}$ the subset of $\mathcal{T}_{E_{v}, K}$ consisting of all elements $\left(\left(b_{1}^{(i)}, b_{2}^{(i)}\right)\right)_{i \in I_{v, K}}$ in $\mathcal{T}_{E_{v}, K}$ such that $b_{1}^{(i)}+b_{2}^{(i)}=e_{v}$ for all $i \in I_{v, K}$.

(iii) Denote by $\boldsymbol{\tau}_{E_{v}, K}$ the set of all sequences

$$
\underline{c}_{v}=\left(c_{v}^{(i)}\right)_{i \in I_{v, K}}, \quad c_{v}^{(i)} \in\left\{0,1, \ldots, e_{v}\right\}, \quad \forall i \in I_{v, K} .
$$

This set $\boldsymbol{\tau}_{E_{v}, K}$ is naturally identified with the set of isomorphism classes of cyclic $\left(\mathcal{O}_{E_{v}} \otimes_{\mathbb{Z}_{p}} K\right)$-modules. 
Remark 3.4.1. (a) Each of the local type spaces $\mathcal{T}_{E_{v}, K}, \mathcal{T}_{E_{v}, K}^{\text {sd }}$ and $\boldsymbol{\tau}_{E_{v}, K}$ has a natural partial ordering, obtained by suppressing the quantifier " $\forall v \in \Sigma_{E, p}$ " in Definition 3.3.

(b) Suppose that $K$ is a field which contains a subfield with $p^{f_{E}}$ elements. Then the finite poset $\mathcal{T}_{E, K}$ (respectively $\mathcal{T}_{E, K}^{\mathrm{sd}}, \boldsymbol{\tau}_{E, K}$ ) is the product, over all $v \in \Sigma_{E, p}$, of the finite posets $\mathcal{T}_{E_{v}, K}$ (respectively $\left.\mathcal{T}_{E_{v}, K}^{\text {sd }}, \boldsymbol{\tau}_{E_{v}, K}\right)$.

(c) We have a local version $\operatorname{strdim}_{v}: \mathcal{T}_{E_{v}, K}^{\text {sd }} \rightarrow \mathbb{N}$ of Definition 3.3.4, given by

$$
\operatorname{strdim}_{v}\left(\left(b_{1}^{(i)}, b_{2}^{(i)}\right)_{i \in I_{v, K}}\right)=\sum_{i \in I_{v, K}}\left(b_{2}^{(i)}-b_{1}^{(i)}\right) .
$$

Then $\operatorname{strdim}(\underline{e})=\sum_{v \in \Sigma_{E, p}} \operatorname{strdim}_{v}\left(\underline{e}_{v}\right)$.

3.5. Newton polygons for abelian varieties with real multiplication

We define a finite poset $\mathrm{NP}_{E}^{\text {sd }}$, which encodes Newton polygons of $g$-dimensional abelian varieties with real multiplication by $\mathcal{O}_{E}$.

Definition 3.5.1. (a) Denote by $\mathrm{NP}_{E}^{\text {sd }}$ the set of all families of pairs $\left(\left(s_{v, 1}, s_{v, 2}\right)\right)_{v \in \Sigma_{E, p}}$ of non-negative rational numbers such that

- $0 \leq s_{v, 1} \leq s_{v, 2} \leq 1$,

- $s_{v, 1}+s_{v, 2}=1$,

- $\left[E_{v}: \mathbb{Q}_{p}\right] \cdot s_{v, 1} \in \mathbb{N}$ and $\left[E_{v}: \mathbb{Q}_{p}\right] \cdot s_{v, 2} \in \mathbb{N}$ whenever $s_{v, 1}<s_{v, 2}$,

for all $v \in \Sigma_{E, p}$.

Elements of $\mathrm{NP}_{E}^{\text {sd }}$ will be called self-dual Newton E-polygons, often shortened to "Newton $E$-polygons" or "Newton polygons" if there is no risk of confusion.

(b) For each element $\underline{s}=\left(\left(s_{v, 1}, s_{v, 2}\right)\right)_{v \in \Sigma_{E, p}}$ in $\mathrm{NP}_{E}^{\text {sd }}$, denote by $\Gamma_{\underline{s}_{v}} \subseteq \mathbb{R}^{2}$ the union of the segment from $(0,0)$ to $\left(\left[E_{v}: \mathbb{Q}_{p}\right],\left[E_{v}: \mathbb{Q}_{p}\right] \cdot s_{v, 1}\right)$ and the segment from $\left(\left[E_{v}: \mathbb{Q}_{p}\right],\left[E_{v}\right.\right.$ : $\left.\left.\mathbb{Q}_{p}\right] \cdot s_{v, 1}\right)$ to $\left(2\left[E_{v}: \mathbb{Q}_{p}\right],\left[E_{v}: \mathbb{Q}_{p}\right]\right)$, for every $v \in \Sigma_{E, p}$. This family $\left(\Gamma_{\underline{s}_{v}}\right)_{v \in \Sigma_{E, p}}$ of polygons in $\mathbb{R}^{2}$ is the more classical form of $\underline{s}$.

Definition 3.5.2. We define a partial ordering on $\mathrm{NP}_{E}^{\mathrm{sd}}$ such that any two elements

$$
\underline{s}=\left(\left(s_{v, 1}, s_{v, 2}\right)\right)_{v \in \Sigma_{E, p}}, \quad \underline{s}^{\prime}=\left(\left(s_{v, 1}^{\prime}, s_{v, 2}^{\prime}\right)\right)_{v \in \Sigma_{E, p}} \in \mathrm{NP}_{E}^{\mathrm{sd}},
$$

we have

$$
\underline{s} \preceq \underline{s}^{\prime} \quad \Longleftrightarrow \quad\left(s_{v, 1} \geq s_{v, 1}^{\prime}, \forall v \in \Sigma_{E, p}\right) .
$$

Equivalently,

$$
\underline{s} \preceq \underline{s}^{\prime} \quad \Longleftrightarrow \quad\left(\Gamma_{\underline{s}_{v}} \text { lies above } \Gamma_{\underline{s}_{v}^{\prime}}, \forall v \in \Sigma_{E, p}\right) .
$$


Remark 3.5.3. (a) The finite poset $\mathrm{NP}_{E}^{\text {sd }}$ is a product of finite posets $\mathrm{NP}_{E_{v}}^{\text {sd }}$ for all $v \in \Sigma_{E, p}$.

(b) For each $v$, the finite poset $\mathrm{NP}_{E_{v}}^{\text {sd }}$ is linearly ordered, and

$$
\operatorname{card}\left(\mathrm{NP}_{E_{v}}^{\mathrm{sd}}\right)=1+\left\lceil\frac{\left[E_{v}: \mathbb{Q}_{p}\right]}{2}\right\rceil .
$$

(c) Let $A$ be a $g$-dimensional abelian variety with real multiplication by $\mathcal{O}_{E}$ over an algebraically closed field $k \supseteq \mathbb{F}_{p}$. For each place $v$ of $E$ above $p$, the rank-two $\mathcal{O}_{E_{v}}$-linear $p$-divisible group $A\left[\wp_{v}^{\infty}\right]$ is either isogenous to direct product of two isoclinic $p$-divisible groups of height $\left[E_{v}: \mathbb{Q}_{p}\right]$, with slopes $s_{v, 1}$ and $s_{v, 2}$, or is isoclinic with slope $1 / 2$. In the latter case we say that $A\left[\wp_{v}^{\infty}\right]$ is supersingular and let $s_{v, 1}=s_{v, 2}=1 / 2$. The element $\left(\left(s_{v, 1}, s_{v, 2}\right)\right)_{v \in \Sigma_{E, p}} \in \mathrm{NP}_{E}^{\text {sd }}$ defined this way is said to be the Newton polygon associated to $A$; cf. Definition 4.2 .5 .

(d) The partial ordering on $\mathrm{NP}_{E}^{\text {sd }}$ is compatible with specialization; see Lemma 4.2.8.

(e) The minimum element in $\mathrm{NP}_{E}^{\text {sd }}$ for the above partial ordering is the element $\underline{s}_{E, \sigma}$ whose $v$-component is $(1 / 2,1 / 2)$ for every $v \in \Sigma_{E, p}$, called the supersingular Newton polygon.

The maximal element in $\mathrm{NP}_{E}^{\text {sd }}$ for the above partial ordering is the element $\underline{s}_{E \text {,ord }}$ whose $v$-component is $(0,1)$ for every $v \in \Sigma_{E, p}$, called the ordinary Newton polygon.

\section{Stratifying Hilbert modular varieties by Lie types}

\subsection{Review of Dieudonné theory}

We use covariant Dieudonné theory for commutative finite group schemes and $p$-divisible groups over perfect fields of characteristic $p$.

Let $\kappa \supseteq \mathbb{F}_{p}$ be a perfect field. Let $\Lambda(\kappa)$ be the ring of $p$-adic Witt vectors with entries in $\kappa$. Let $\sigma$ be the continuous automorphism of $\Lambda(\kappa)$ which sends every element $\left(a_{0}, a_{1}, \ldots, a_{n}, \ldots\right) \in \Lambda(\kappa)$ to $\left(a_{0}^{p}, a_{1}^{p}, \ldots, a_{n}^{p}, \ldots\right)$.

Let $R_{\kappa}$ be the (non-commutative) ring generated by $\Lambda(\kappa)$ and elements $\mathcal{F}, \mathcal{V}$, with defining relations

$$
\mathcal{F} a={ }^{\sigma} a \mathcal{F}, \quad a \mathcal{V}=\mathcal{V}^{\sigma} a, \quad \forall a \in \Lambda(\kappa) \quad \text { and } \quad \mathcal{F} \mathcal{V}=\mathcal{V} \mathcal{F}=p
$$

Let $\boldsymbol{C F G} \boldsymbol{S}_{\kappa}$ be the category of commutative finite group schemes over $\kappa$, and let $\boldsymbol{D} \boldsymbol{M}_{\kappa, \mathrm{f}}$ be the category of modules over $R_{\kappa}$ which are of finite length as modules over $\Lambda(\kappa)$.

Denote by $p$ - $\boldsymbol{D i v _ { \kappa }}$ the category of $p$-divisible groups over $\kappa$, and denote by $\boldsymbol{D} \boldsymbol{M}_{\kappa}$ the category of modules over $R_{\kappa}$ which are free of finite rank as modules over $\Lambda(\kappa)$.

4.1.1. We have an exact covariant additive functor

$$
\mathbb{D}: \boldsymbol{C F G} \boldsymbol{S}_{\kappa} \rightarrow \boldsymbol{D} M_{\kappa, \mathrm{f}}
$$


which is an equivalence of abelian categories and satisfies the following properties.

(i) For every commutative finite group scheme $G$ over $\kappa$, with relative Frobenius and Verschiebung homomorphisms

$$
\operatorname{Fr}_{G / \kappa}: G \rightarrow G^{(p)}:=G \times\left(\operatorname{Spec}(\kappa), \operatorname{Fr}_{\kappa}^{*}\right) \operatorname{Spec}(\kappa) \text { and } \operatorname{Ver}_{G / \kappa}: G^{(p)} \rightarrow G,
$$

the operator $\mathcal{V}_{G}$ on $\mathbb{D}(G)$ is equal to the composition

$$
\mathbb{D}(G) \stackrel{\mathbb{D}\left(\operatorname{Fr}_{G / \kappa}\right)}{\longrightarrow} \mathbb{D}\left(G^{(p)}\right) \stackrel{=}{\longrightarrow} \Lambda(\kappa) \otimes_{(\sigma, \Lambda(\kappa))} \mathbb{D}(G) \stackrel{\sigma^{-1} \otimes 1_{\mathbb{D}(G)}}{\longrightarrow} \mathbb{D}(G),
$$

and the operator $\mathcal{F}_{G}$ is equal to the composition

$$
\mathbb{D}(G) \stackrel{x \mapsto 1 \otimes x}{\longrightarrow} \Lambda(\kappa) \otimes_{(\sigma, \Lambda(\kappa))} \mathbb{D}(G) \stackrel{=}{\longrightarrow} \mathbb{D}\left(G^{(p)}\right) \stackrel{\mathbb{D}\left(\operatorname{Ver}_{G / \kappa}\right)}{\longrightarrow} \mathbb{D}(G) .
$$

(ii) A commutative finite group scheme $G$ over $\kappa$ is etale (respectively multiplicative) if and only if $\mathcal{V}_{G}$ (respectively $\mathcal{F}_{G}$ ) is bijective.

(iii) If the relative Frobenius homomorphism

$$
\operatorname{Fr}_{G / \kappa}: G \rightarrow G^{(p)}
$$

is 0 , then we have a natural isomorphism

$$
\mathbb{D}(G)=\operatorname{Lie}(G)^{(p)}:=\operatorname{Lie}(G) \otimes_{\left(\kappa, \operatorname{Fr}_{\kappa}\right)} \kappa .
$$

4.1.2. The Dieudonné functor $\mathbb{D}$ in Subsubsection 4.1.1 induces an exact additive functor

$$
\mathbb{D}: p-\boldsymbol{D i v} \boldsymbol{v}_{\kappa} \rightarrow \boldsymbol{D} \boldsymbol{M}_{\kappa}
$$

which is an equivalence of categories with the following properties.

(i) For every $p$-divisible group $X$ over $\kappa$ and for every $m \in \mathbb{N}$, we have a functorial isomorphism

$$
p^{-m} \mathbb{D}(X) / \mathbb{D}(X) \stackrel{\sim}{\rightarrow} \mathbb{D}\left(X\left[p^{m}\right]\right)
$$

of $R_{\kappa}$-modules.

(ii) The operators $\mathcal{V}_{X}, \mathcal{F}_{X}$ on the Dieudonné module $\mathbb{D}(X)$ of a $p$-divisible group $X$ over $\kappa$ are equal to the compositions

$$
\mathbb{D}(X) \stackrel{\mathbb{D}\left(\operatorname{Fr}_{X / \kappa}\right)}{\longrightarrow} \mathbb{D}\left(X^{(p)}\right) \stackrel{=}{\longrightarrow} \Lambda(\kappa) \otimes_{(\sigma, \Lambda(\kappa))} \mathbb{D}(X) \stackrel{\sigma^{-1} \otimes 1_{\mathbb{D}(X)}}{\longrightarrow} \mathbb{D}(X)
$$

and

$$
\mathbb{D}(X) \stackrel{x \mapsto 1 \otimes x}{\longrightarrow} \Lambda(\kappa) \otimes_{(\sigma, \Lambda(\kappa))} \mathbb{D}(X) \stackrel{=}{\longrightarrow} \mathbb{D}\left(X^{(p)}\right) \stackrel{\mathbb{D}\left(\operatorname{Ver}_{X / \kappa}\right)}{\longrightarrow} \mathbb{D}(X)
$$

respectively. 
(iii) A $p$-divisible group $X$ over $\kappa$ is etale (respectively multiplicative) if and only if $\mathcal{V}_{X}$ (respectively $\mathcal{F}_{X}$ ) is bijective.

(iv) For every $p$-divisible group $X$ over $\kappa$ we have natural isomorphisms

$$
\operatorname{Lie}(X) \stackrel{\sim}{\rightarrow} \mathbb{D}(X) / \mathcal{V}(\mathbb{D}(X)) \quad \text { and } \quad H_{1}^{\mathrm{dR}}(X / \kappa) \stackrel{\sim}{\rightarrow} \mathbb{D}(X) / p \mathbb{D}(X),
$$

so that the short exact sequence

$$
0 \rightarrow \mathcal{V}(\mathbb{D}(X)) / p \mathbb{D}(X) \rightarrow \mathbb{D}(X) / p \mathbb{D}(X) \rightarrow \mathbb{D}(X) / \mathcal{V}(\mathbb{D}(X)) \rightarrow 0
$$

is identified with the Hodge filtration of the first de Rham homology group $H_{1}^{\mathrm{dR}}(X / \kappa)$ of $X$.

The formulas in Lemma 4.1.3 below have already been stated in Subsubsections 4.1.1(iii) and 4.1.2(iv). We give an explicit isomorphism $\mathbb{D}(X[F]) \cong \operatorname{Lie}(X[F])^{(p)}$ as an illustration.

Lemma 4.1.3. Let $X$ be a $p$-divisible group over a perfect field $\kappa \supseteq \mathbb{F}_{p}$, and let $M:=\mathbb{D}(X)$ be the Dieudonné module of $X$. Let $X[F]=\operatorname{Ker}\left(\operatorname{Fr}_{X / \kappa}: X \rightarrow X^{(p)}\right)$ be the kernel of relative Frobenius homomorphism of $X$. We have natural $\kappa$-linear isomorphisms

$$
\operatorname{Lie}(X) \cong M / \mathcal{V} M
$$

and

$$
\mathbb{D}(X[F]) \underset{\cong}{\simeq} p^{-1} \mathcal{F}(M) / M \stackrel{\mathbb{D}\left(\operatorname{Ver}_{X}\right)}{\simeq}(M / \mathcal{V}(M))^{(p)} \underset{\cong}{\longrightarrow} \operatorname{Lie}(X)^{(p)} .
$$

Proof. The covariant Dieudonné theory we used is normalized so that the Lie algebra $\operatorname{Lie}(X)$ of $X$ is naturally isomorphic to $\mathbb{D}(X) / \mathcal{V}(\mathbb{D}(X))$. The isomorphism

$$
\mathbb{D}(X[F]) \stackrel{\cong}{\longrightarrow} p^{-1} \mathcal{F}(M) / M
$$

follows from Subsubsection 4.1.2(i) and the exactness of the Dieudonné functor for commutative finite group schemes over $\kappa$, because

$$
p^{-1} \mathcal{F}(M) / M=\operatorname{Ker}\left(p^{-1} M / M \stackrel{\mathcal{V}}{\rightarrow} p^{-1} M / M\right) .
$$

Clearly the $\sigma^{-1}$-linear operator $\mathcal{V}$ on $p^{-1} M / M$ induces a $\kappa$-linear isomorphism

$$
\left(p^{-1} \mathcal{F}(M) / M\right)^{\left(p^{-1}\right)} \stackrel{\simeq}{\longrightarrow} M / \mathcal{V}(M)
$$

Remark 4.1.4. (a) There are (at least) two versions of covariant Dieudonné theory, which differ from each other by a Frobenius twist. The properties in Subsubsections 4.1.1(iii) and 4.1.2(iv) are "normalization conditions" which specify the Dieudonné functors $G \rightsquigarrow \mathbb{D}(G)$ and $X \rightsquigarrow \mathbb{D}(X)$ in Subsubsections 4.1.1 and 4.1.2. Note also that the statement of 
Lemma 4.1 .3 will need to be modified by a Frobenius twist if a different Dieudonné theory is used.

(b) The functor $G \rightsquigarrow \mathbb{D}(G)$ used here is the covariant crystalline Dieudonné theory, denoted by $G \rightsquigarrow \mathrm{E}_{*}(G)$ in [8, B.3].

We refer to [8, B.3] for the literature and further information about Dieudonné theory, including the formulas for basic differential invariants in [8, B.3.11] and the comparison with the classical Dieudonné theory in [8, B.3.10].

4.2. Rank-two $\mathcal{O}_{E}$-linear $p$-divisible groups and their Dieudonné modules

We apply Dieudonné theory to the $p$-divisible groups associated to abelian varieties with real multiplication by $\mathcal{O}_{E}$.

Definition 4.2.1. Let $S$ be an $\mathbb{F}_{p}$-scheme.

(a) Let $v$ be a place of $E$ above $p$. A rank-two $\mathcal{O}_{E_{v}}$-linear $p$-divisible group over $S$ is a pair $\left(X_{v} \rightarrow S, \iota_{v}\right)$, where $X_{v} \rightarrow S$ is a $p$-divisible group over $S$ of height $2\left[E_{v}: \mathbb{Q}_{p}\right]$, and $\iota_{v}: \mathcal{O}_{E_{v}} \rightarrow \operatorname{End}_{S}\left(X_{v}\right)$ is a ring homomorphism.

(b) A rank-two $\mathcal{O}_{E}$-linear $p$-divisible group over $S$ is a pair $(X \rightarrow S, \iota)$ where $X \rightarrow S$ is a $p$-divisible group and $\iota: \mathcal{O}_{E} \otimes_{\mathbb{Z}} \mathbb{Z}_{p} \rightarrow \operatorname{End}_{S}(X)$ is a ring homomorphism, such that for every $v \in \Sigma_{E, p}$, the $p$-divisible group $X\left[\wp_{v}^{\infty}\right] \rightarrow S$ has height $2\left[E_{v}: \mathbb{Q}_{p}\right]$. In other words, there exist rank-two $\mathcal{O}_{E_{v}}$-linear $p$-divisible groups $\left(X_{v} \rightarrow S, \iota_{v}\right), v \in \Sigma_{E, p}$, such that the fiber product over $S$ of the $\left(X_{v}, \iota_{v}\right)$ 's is $\left(\mathcal{O}_{E} \otimes_{\mathbb{Z}} \mathbb{Z}_{p}\right)$-linearly isomorphic to $(X, \iota)$.

(c) A rank-two $\mathcal{O}_{E_{v}}$-linear $p$-divisible group $\left(X_{v} \rightarrow S, \iota_{v}\right)$ is said to be self-dual if its Serre dual $\left(X_{v}^{t} \rightarrow S, \iota^{t}\right)$ is $\mathcal{O}_{E_{v}}$-linearly isomorphic to itself. A rank-two $\mathcal{O}_{E}$-linear $p$-divisible group $(X \rightarrow S, \iota)$ is self-dual if its Serre dual $\left(X^{t} \rightarrow S, \iota^{t}\right)$ is $\mathcal{O}_{E^{-}}$-linearly isomorphic to itself.

(d) A rank-two $\mathcal{O}_{E_{v}}$-linear $p$-divisible group $\left(X_{v} \rightarrow S, \iota_{v}\right)$ is said to be self-dual up to isogeny if there exists an $\mathcal{O}_{E_{v}}$-linear $S$-isogeny from $\left(X_{v}, \iota_{v}\right)$ to $\left(X_{v}^{t}, \iota_{v}^{t}\right)$. A rank-two $\mathcal{O}_{E}$-linear $p$-divisible group $(X \rightarrow S, \iota)$ is self-dual up to isogeny if there exists an $\mathcal{O}_{E}$-linear isogeny over $S$ from $X$ to $X^{t}$.

Lemma 4.2.2. Let $(A, \iota)$ be an abelian variety with real multiplication by $\mathcal{O}_{E}$ over a field $K \supseteq \mathbb{F}_{p}$.

(a) The $p$-divisible group $A\left[p^{\infty}\right]$ with action by $\mathcal{O}_{E} \otimes_{\mathbb{Z}} \mathbb{Z}_{p}$ is a rank-two $\mathcal{O}_{E}$-linear $p$ divisible group which is self-dual up to isogeny. 
(b) If $(A, \iota)$ admits a principal $\widetilde{\mathscr{L}}$-polarization, then the rank-two $\mathcal{O}_{E}$-linear $p$-divisible group $\left(A\left[p^{\infty}\right], \iota\left[p^{\infty}\right]\right)$ is self-dual.

Lemma 4.2.3. Let $(X, \iota)$ be a self-dual rank-two $\mathcal{O}_{E}$-linear p-divisible group over a field $K \supset \mathbb{F}_{p}$, then the isomorphism class in $\boldsymbol{T}_{E, K}$ of the Lie algebra Lie $(X, \iota)$ of $(X, \iota)$ is self-dual; i.e., the class $[\operatorname{Lie}(X, \iota)]$ of $\operatorname{Lie}(X, \iota)$ in the $K$-group of finitely generated $\left(\left(\mathcal{O}_{E} / p \mathcal{O}_{E}\right) \otimes_{\mathbb{F}_{p}} K\right)$-modules is equal to the class $\left[\left(\mathcal{O}_{E} / p \mathcal{O}_{E}\right) \otimes_{\mathbb{F}_{p}} K\right]$ of $\left(\mathcal{O}_{E} / p \mathcal{O}_{E}\right) \otimes_{\mathbb{F}_{p}} K$.

Lemma 4.2.4. Let $(X, \iota)$ be a rank-two $\mathcal{O}_{E}$-linear $p$-divisible group over a field $K \supseteq \mathbb{F}_{p}$ which is self-dual up to isogeny. For every $v \in \Sigma_{E, p}$, suppose that the p-divisible group $X\left[\wp_{v}^{\infty}\right]$ is not isoclinic of slope $1 / 2$. Then $X\left[\wp_{v}^{\infty}\right]$ is isogenous to the product of two isoclinic $\mathcal{O}_{E_{v}}$-linear $p$-divisible groups $Y_{1}, Y_{2}$ of height $\left[E_{v}: \mathbb{Q}_{p}\right]$, and the slopes $s_{v, 1}, s_{v, 2}$ of $Y_{1}, Y_{2}$ satisfy

$$
0 \leq s_{v, 1}, s_{v, 2} \leq 1, \quad s_{v, 1}+s_{v, 2}=1 \quad \text { and } \quad\left[E_{v}: \mathbb{Q}_{p}\right] \cdot s_{v, i} \in \mathbb{N}
$$

Definition 4.2.5. (a) Let $(X, \iota)$ be a rank-two $\mathcal{O}_{E}$-linear $p$-divisible group over a field $K \supseteq \mathbb{F}_{p}$ which is self-dual up to isogeny. The Newton polygon of $(X, \iota)$ is the element $\left(\left(s_{v, 1}, s_{v, 2}\right)\right)_{v \in \Sigma_{E, p}} \in \mathrm{NP}_{E}^{\text {sd }}$ such that

$$
\left(s_{v, 1}, s_{v, 2}\right)= \begin{cases}\left(s_{X, v, 1}, s_{X, v, 2}\right) & \text { if } X\left[\wp_{v}^{\infty}\right] \text { has two distinct slopes } s_{X, v, 1}<s_{X, v, 2}, \\ (1 / 2,1 / 2) & \text { if } X\left[\wp_{v}^{\infty}\right] \text { is isoclinic. }\end{cases}
$$

(b) Let $(A, \iota)$ be an abelian variety with real multiplication by $\mathcal{O}_{E}$ over a field $K \supseteq \mathbb{F}_{p}$. The Newton polygon of $(A, \iota)$ is by definition the Newton polygon of $\left(A\left[p^{\infty}\right], \iota\left[p^{\infty}\right]\right)$.

Definition 4.2.6. For each $\underline{s} \in \mathrm{NP}_{E}^{\text {sd }}$, denote by $\mathcal{W}_{\underline{s}}=\mathcal{W}_{\underline{s}}\left(\mathscr{M}_{E}\right)$ the locally closed subset of $\mathscr{M}_{E}$ consisting of all points $x \in \mathscr{M}_{E}$ whose Newton polygon is equal to $\underline{s}$. Clearly $\mathscr{M}_{E}$ is the disjoint union of all Newton polygon strata $\mathcal{W}_{\underline{s}}$, where $\underline{s}$ runs through all elements of $\mathrm{NP}_{E}^{\mathrm{sd}}$.

Remark. The Newton polygon stratum $\mathcal{W}_{\underline{s}_{E, \sigma}}$ corresponding to the minimal element $\underline{s}_{E, \sigma}$ in $\mathrm{NP}_{E}^{\text {sd }}$, called the supersingular locus in $\mathscr{M}_{E}$, is the closed subset of $\mathscr{M}_{E}$ consisting of points whose underlying abelian varieties are isoclinic of slope $1 / 2$.

Remark 4.2.7. There is another notion of Newton polygon strata attached to a Newton polygon $\underline{s}$ in the literature. What was defined in Definition 4.2.5(a) is sometimes called the open Newton polygon stratum attached to $\underline{s}$ and denoted by $\mathcal{W}_{\underline{s}}^{0}$.

Lemma 4.2.8. Let $S$ be a scheme over $\mathbb{F}_{p}$ and let $(X \rightarrow S, \iota)$ be a rank-two $\mathcal{O}_{E}$-linear p-divisible group with real multiplication by $\mathcal{O}_{E}$ which is self-dual up to isogeny. Let $z, z^{\prime}$ be points of $S$, and let $\underline{s}_{z}, \underline{s}_{z^{\prime}} \in \mathrm{NP}_{E}^{\text {sd }}$ be the Newton -polygons of $X_{z}$ and $X_{z^{\prime}}$ respectively. If $z$ is a specialization of $z^{\prime}$, then $\underline{s}_{z} \preceq \underline{s}_{z^{\prime}}$. 
Corollary 4.2.9. Let $\underline{s}$ be an element of $\mathrm{NP}_{E}^{\mathrm{sd}}$. The Zariski closure $\left(\mathcal{W}_{\underline{s}}\right)^{\mathrm{zar}}$ of the Newton polygon stratum $\mathcal{W}_{\underline{s}}$ in $\mathscr{M}_{E}$ is contained in the union of all Newton polygon strata $\mathcal{W}_{\underline{s}^{\prime}}$ with $\underline{s}^{\prime} \preceq \underline{s}$ :

$$
\left(\mathcal{W}_{\underline{s}}\right)^{\mathrm{zar}} \subseteq \bigsqcup_{\underline{s}^{\prime} \preceq \underline{s}} \mathcal{W}_{\underline{s}^{\prime}} .
$$

Remark. Whether $\left(\mathcal{W}_{\underline{s}}\right)^{\text {zar }}$ is equal to $\bigsqcup_{\underline{s}^{\prime} \preceq \underline{s}} \mathcal{W}_{\underline{s}^{\prime}}$ is the content of the Grothendieck conjecture on the Newton polygon stratification of $\mathscr{M}_{E}$; cf. Question 10.4 (d)-(e).

Definition 4.2.10. Let $\kappa$ be a field which contains a subfield with $p^{f_{E}}$ elements. Let $\kappa_{1}=\kappa^{\text {perf }}$ be the perfection of $\kappa$.

(a) Let $v$ be a place of $E$ above $p$ and let $\left(X_{v}, \iota_{v}\right)$ be a rank-two $\mathcal{O}_{E_{v}}$-linear $p$-divisible group over $\kappa$. The Lie type of $\left(X_{v}, \iota_{v}\right)$ is the element $\underline{e}_{X_{v}} \in \mathcal{T}_{E_{v}}$ which corresponds to the isomorphism class of the $\left(\mathcal{O}_{E_{v}} \otimes_{\mathbb{Z}_{p}} \kappa\right)$-module Lie $\left(X_{v}\right)$. Equivalently, $\underline{e}_{X_{v}}$ is the element of $\mathcal{T}_{E_{v}, \kappa}$ whose image in $\mathcal{T}_{E_{v}, \kappa_{1}}$ under the bijection $\mathcal{T}_{E_{v}, \kappa} \stackrel{\sim}{\rightarrow} \mathcal{T}_{E_{v}, \kappa_{1}}$ is the isomorphism class of $\mathbb{D}\left(X_{v} \times_{\operatorname{Spec}(\kappa)} \operatorname{Spec}\left(\kappa_{1}\right)\right) / \mathcal{V} \cdot \mathbb{D}\left(X_{v} \times_{\operatorname{Spec}(\kappa)} \operatorname{Spec}\left(\kappa_{1}\right)\right)$.

(b) Let $(X, \iota)$ be a rank-two $\mathcal{O}_{E}$-linear $p$-divisible group over $\kappa$. The Lie type of $(X, \iota)$ is the element $\underline{e}_{X} \in \mathcal{T}_{E}$ which corresponds to the isomorphism class of the $\left(\mathcal{O}_{E} \otimes_{\mathbb{Z}} \kappa\right)$ module Lie $(X)$. In other words $\underline{e}_{X}=\left(\underline{e}_{X_{v}}\right)_{v \in \Sigma_{E, p}}$, where $\underline{e}_{X_{v}}$ is the Lie type of the rank-two $\mathcal{O}_{E_{v}}$-linear $p$-divisible group $X_{v}=X\left[\wp_{v}^{\infty}\right]$, for every place $v$ of $E$ above $p$.

(c) Let $(A, \iota)$ be an abelian variety with real multiplication by $\mathcal{O}_{E}$. The Lie type $\underline{e}_{A}$ of $(A, \iota)$ is by definition the Lie type of the rank-two $\mathcal{O}_{E}$-linear $p$-divisible group $\left(A\left[p^{\infty}\right], \iota\left[p^{\infty}\right]\right)$.

Lemma 4.2.11. Let $k \supseteq \mathbb{F}_{p}$ be an algebraically closed field, and let $(X, \iota)$ be a rank-two $\mathcal{O}_{E}$-linear p-divisible group. Let $\underline{e}_{X}=\left(e_{X, v, 1}^{(i)}, e_{X, v, 2}^{(i)}\right)_{v \in \Sigma_{E, p}, i \in I_{v}} \in \mathcal{T}_{E}$ be the Lie type of $(X, \iota)$. Then $(X, \iota)$ is self-dual if and only if $\underline{e}_{X} \in \mathcal{T}_{E}^{\mathrm{sd}}$, i.e., $e_{X, v, 1}^{(i)}+e_{X, v, 2}^{(i)}=e_{v}$ for all $v \in \Sigma_{E, p}$ and all $i \in I_{v}$.

The definition and properties of Lie stratification of Hilbert modular varieties, due to Deligne and Pappas in [13, Sections 3-4], is summarized in Theorem 4.3 below.

Theorem 4.3. For each $\underline{e} \in \mathcal{T}_{E}^{\mathrm{sd}}$, there exists a locally closed subscheme

$$
\mathcal{N}_{\underline{e}}\left(\mathscr{M}_{E}\right) \subseteq \mathscr{M}_{E}=\mathscr{M}_{E, n, \bar{\delta}}^{\widetilde{\mathscr{L}}}
$$

over $\overline{\mathbb{F}_{p}}$, characterized by the property that for every point $x \in \mathscr{M}_{E}$, we have

$$
x \in \mathcal{N}_{\underline{e}}\left(\mathscr{M}_{E}\right) \quad \Longleftrightarrow \quad \underline{e}_{\left(A_{x}, \iota_{x}\right)}=\underline{e} .
$$

Here $\left(A_{x}, \iota_{x}\right)$ denotes the fiber at $x$ of the universal abelian scheme with real multiplication by $\mathcal{O}_{E}$ over $\mathscr{M}_{E}$. The subvarieties $\mathcal{N}_{\underline{e}}=\mathcal{N}_{\underline{e}}\left(\mathscr{M}_{E}\right)$ satisfy the following properties: 
(1) The modular variety $\mathscr{M}_{E}$ is the disjoint union of these Lie strata:

$$
\mathscr{M}_{E}=\bigsqcup_{\underline{e} \in \mathcal{T}_{E}^{\mathrm{sd}}} \mathcal{N}_{\underline{e}}\left(\mathscr{M}_{E}\right)=\bigsqcup_{\underline{e} \in \mathcal{T}_{E}^{\mathrm{sd}}} \mathcal{N}_{\underline{e}} .
$$

(2) Each Lie stratum $\mathcal{N}_{\underline{e}}$ is a locally closed smooth subscheme of $\mathscr{M}_{E}$.

(3) The dimension of the Lie stratum $\mathcal{N}_{\underline{e}}$ indexed by an element

$$
\underline{e}=\left(\left(e_{v, 1}^{(i)}, e_{v, 2}^{(i)}\right)_{i \in I_{v}}\right)_{v \in \Sigma_{E, p}} \in \mathcal{T}_{E}^{\mathrm{sd}}
$$

is given by

$$
\operatorname{dim}\left(\mathcal{N}_{\underline{e}}\right)=\operatorname{strdim}(\underline{e})=\sum_{v} \sum_{i \in I_{v}}\left(e_{v, 2}^{(i)}-e_{v, 1}^{(i)}\right) .
$$

In particular the dimension of the minimal Lie stratum $\mathcal{N}_{\min }=\mathcal{N}_{\underline{e}_{\min }}\left(\mathscr{M}_{E}\right)$ is

$$
\operatorname{dim}\left(\mathcal{N}_{\text {min }}\right)=\sum_{e_{v} \text { odd }} f_{v}
$$

where the sum runs through all places $v$ of $E$ above $p$ with odd ramification index $e_{v}$, and $f_{v}=\left[\kappa_{v}: \mathbb{F}_{p}\right]$ is the degree over $\mathbb{F}_{p}$ of the residue field $\kappa_{v}$ of $\mathcal{O}_{E_{v}}$.

(4) The maximal Lie stratum $\mathcal{N}_{\max }=\mathcal{N}_{\underline{e}_{\max }}\left(\mathscr{M}_{E}\right)$ coincides with the smooth locus of $\mathscr{M}_{E}$, i.e., the largest open subset of $\mathscr{M}_{E}$ which is smooth over $\overline{\mathbb{F}_{p}}$.

(5) The Zariski closure $\left(\mathcal{N}_{\underline{e}}\right)^{\mathrm{zar}}$ of a Lie stratum $\mathcal{N}_{\underline{e}}$ in $\mathscr{M}_{E}$ is the disjoint union of all strata $\mathcal{N}_{\underline{e}^{\prime}}$, where $\underline{e}^{\prime}$ runs through all elements in $\mathcal{T}_{E}^{\mathrm{sd}}$ such that $\underline{e}^{\prime} \preceq \underline{e}$, i.e.,

$$
\left(\mathcal{N}_{\underline{e}}\right)^{\mathrm{zar}}=\bigsqcup_{\underline{e}^{\prime} \preceq \underline{e}} \mathcal{N}_{\underline{e}^{\prime}} .
$$

(6) For each $\underline{e} \in \mathcal{T}_{E}^{\mathrm{sd}},\left(\mathcal{N}_{\underline{e}}\right)^{\mathrm{zar}}$ is a local complete intersection and is normal, and $\mathcal{N}_{\underline{e}}$ is the largest open subset of $\left(\mathcal{N}_{\underline{e}}\right)^{\text {zar }}$ which is smooth over $\overline{\mathbb{F}_{p}}$.

(7) Suppose that $\underline{e}_{1} \supsetneqq \underline{e}_{2}$ are elements of $\mathcal{T}_{E}^{\mathrm{sd}}$, and there is no element $\underline{e} \in \mathcal{T}_{E}^{\mathrm{sd}}$ such that $\underline{e}_{1} \supsetneqq \underline{e} \supsetneqq \underline{e}_{2}$, then $\operatorname{dim}\left(\mathcal{N}_{\underline{e}_{1}}\right)=\operatorname{dim}\left(\mathcal{N}_{\underline{e}_{2}}\right)-2$.

\section{The congruity invariant}

In this section we define a numerical invariant, called congruity, attached to every ranktwo $\mathcal{O}_{E}$-linear $p$-divisible group $(X, \iota)$ over an algebraically closed field $k$ of characteristic $p$. When the congruity invariant $c_{(X, \iota)}$ takes certain specific values, the isomorphism class of $(X, \iota)$ is uniquely determined by the invariant $c_{(X, \iota)}$, in which case $(X, \iota)$ is said to be 
distinguished; see Definitions 5.3.2, 5.4.3 and 5.6. The definition of the congruity invariant is described in the next two paragraphs.

In Definition 5.1 .1 we define an $\mathbb{N}$-valued invariant $c\left(M ; N, N^{\prime}\right)$ associated to an unordered pair $\left(N, N^{\prime}\right)$ of two lattices in a rank-two module $M$ over a discrete valuation ring, which measures the extent the of the failure of these two lattices in $M$ to be transversal up to rescaling inside $M$.

For a rank-two $\mathcal{O}_{E}$-linear $p$-divisible group $(X, \iota)$ over a sufficiently large perfect field $\kappa_{1}$ of characteristic $p$, the Dieudonné module $\mathbb{D}(X)$ is a projective rank-two module over $\mathcal{O}_{E} \otimes_{\mathbb{Z}} \Lambda\left(\kappa_{1}\right)$, which decomposes into a direct sum, indexed by the disjoint union of the sets $I_{v}$, of rank-two projective modules $\mathbb{D}(X)_{v}^{(i)}$ over discrete valuation rings $\mathcal{O}_{E_{v}} \otimes_{\left(\mathcal{O}_{E_{v}}, i\right)} \Lambda\left(\kappa_{1}\right)$. The same holds for $\mathcal{F}(\mathbb{D}(X))$ and $\mathcal{V}(\mathbb{D}(X))$. For each place $v$ of $E$ above $p$ and each $i \in I_{v}$, the construction in Definition 5.1.1 applied to $\mathbb{D}(X)_{v}^{(i)},(\mathcal{F} \mathbb{D}(X))_{v}^{(i)}$ and a "transport" to $\mathbb{D}(X)_{v}^{(i)}$ of $(\mathcal{V D}(X))_{v}^{\left(i^{\prime}\right)}$ for a suitable element $i^{\prime} \in I_{v}$, gives the $(v, i)$-component of the congruity invariant $\underline{c}_{(X, \iota)}$ of $(X, \iota)$ defined in Definition 5.2 .

\subsection{Lattices in a free rank-two module over a discrete valuation ring}

In this subsection, $D$ denotes a discrete valuation ring with maximal ideal $\wp$ and residue field $\kappa=D / \wp$. Let $M$ be a free rank-two $D$-module. We will define a discrete invariant $c\left(M ; N, N^{\prime}\right)$ attached to two lattices $N, N^{\prime}$ in $M$.

Recall that the elementary divisors $\left(b_{1}, b_{2}\right)$ of a lattice $N$ in $M, b_{1} \leq b_{2}$, are the two natural numbers determined by $M / N \cong D / \wp^{b_{1}} \oplus D / \wp^{b_{2}}$, or equivalently there exists a $D$-basis $v_{1}, v_{2} \in M$ such that $N=\wp^{b_{1}} \cdot v_{1}+\wp^{b_{2}} \cdot v_{2}$. Note that $b_{1}=\max \left\{b \mid N \subseteq \wp^{b} M\right\}$, and $b_{2}=\min \left\{b \mid N \supseteq \wp^{b} M\right\}$.

Definition 5.1.1 (An invariant of two lattices in $D^{2}$ ). Let $N, N^{\prime}$ be two lattices in $M$, with elementary divisors $\left(b_{1}, b_{2}\right)$ and $\left(b_{1}^{\prime}, b_{2}^{\prime}\right)$ respectively, $b_{1} \leq b_{2}, b_{1}^{\prime} \leq b_{2}$. Define an invariant $c\left(M ; N, N^{\prime}\right)$ by

$$
M /\left(\wp^{-b_{1}} N+\wp^{-b_{1}^{\prime}} N^{\prime}\right) \cong D / \wp^{c\left(M ; N, N^{\prime}\right)} .
$$

Note that the elementary divisors of $\wp^{-b_{1}} N$ and $\wp^{-b_{1}^{\prime}} N^{\prime}$ are $\left(0, b_{2}-b_{1}\right)$ and $\left(0, b_{2}^{\prime}-b_{1}^{\prime}\right)$ respectively. So $M /\left(\wp^{-b_{1}} N+\wp^{-b_{1}^{\prime}} N^{\prime}\right)$ is a cyclic $D$-module of finite length. Note also that the equality

$$
0 \leq c\left(M ; N, N^{\prime}\right) \leq \min \left\{b_{2}-b_{1}, b_{2}^{\prime}-b_{1}^{\prime}\right\}
$$

holds.

Remark 5.1.2. The congruity invariant can be computed as follows. Let $\left(x_{1}, x_{2}\right)$ be a $D$-basis of $M$ such that $N=\wp^{b_{1}} x_{1}+\wp^{b_{2}} x_{2}$, and let $\left(y_{1}, y_{2}\right)$ be a $D$-basis of $M$ such that 
$N^{\prime}=\wp^{b_{1}^{\prime}} y_{1}+\wp^{b_{2}^{\prime}} y_{2}$. Define an element $\left(a_{i j}\right)_{i, j=1,2} \in \mathrm{GL}_{2}(D)$ by

$$
y_{j}=\sum_{i=1,2} a_{i j} x_{i}, \quad j=1,2 \text {. }
$$

From

$$
\begin{aligned}
\wp^{-b_{1}} N+\wp^{-b_{1}^{\prime}} N^{\prime} & =D x_{1}+D \cdot a_{21} x_{2}+\wp^{b_{2}-b_{1}} x_{2}+\wp^{b_{2}^{\prime}-b_{1}^{\prime}} y_{2} \\
& =D y_{1}+D \cdot a_{21} y_{2}+\wp^{b_{2}-b_{1}} x_{2}+\wp^{b_{2}^{\prime}-b_{1}^{\prime}} y_{2},
\end{aligned}
$$

we see that

$$
c\left(M ; N, N^{\prime}\right)=\min \left\{\operatorname{ord}_{\wp}\left(a_{21}\right), b_{2}-b_{1}, b_{2}^{\prime}-b_{1}^{\prime}\right\} .
$$

Definition 5.1.3. Let $\underline{e}_{X}=\left(\underline{e}_{v}\right)_{v \in \Sigma_{E, p}}=\left(\left(e_{v, 1}^{(i)}, e_{v, 2}^{(i)}\right)_{i \in I_{v}}\right)_{v \in \Sigma_{E, p}}$ be an element of $\mathcal{T}_{E}^{\mathrm{sd}}$. For every $v \in \Sigma_{E, p}$, define a function $t_{\underline{e}_{v}}: I_{v} \rightarrow \mathbb{N} \cup\{-\infty\}$ by

$$
t_{\underline{e}_{v}}(i):=\min \left\{n \in \mathbb{N} \mid e_{1}^{(i+n)}<e_{2}^{(i+n)}\right\}, \quad \forall i \in I_{v} .
$$

Note that $t_{\underline{e}_{v}}(i)=-\infty$ if and only if the set $\left\{n \in \mathbb{N} \mid e_{1}^{(i+n)}<e_{2}^{(i+n)}\right\}$ is empty, in which case the function $t_{\underline{e}_{v}}$ on $I_{v}$ has constant value $-\infty$. Note also that $t_{\underline{e}_{v}}(i)=0$ if and only if $e_{v, 1}^{(i)}<e_{v, 2}^{(i)}$.

Definition 5.2 (Congruity). Let $\kappa$ be a field which contains a finite subfield with $p^{f_{E}}$ elements. Let $\kappa_{1}=\kappa^{\text {perf }}$ be the perfection of $\kappa$.

(a) Let $v$ be a place of $E$ above $p$. Let $(X, \iota)$ be a rank-two $\mathcal{O}_{E}$-linear $p$-divisible group over $\kappa$. Let $M_{v}:=\mathbb{D}\left(X_{v} \times_{\operatorname{Spec}(\kappa)} \operatorname{Spec}\left(\kappa_{1}\right)\right)$ be the covariant Dieudonné module of $X_{v} \times_{\operatorname{Spec}(\kappa)} \operatorname{Spec}\left(\kappa_{1}\right)$. Let

$$
M_{v}=\bigoplus_{i \in I_{v}} M_{v}^{(i)}
$$

be the decomposition of $M_{v}$ corresponding to the product decomposition of the ring $\mathcal{O}_{E_{v}} \otimes_{\mathbb{Z}_{p}} \Lambda(\kappa)$ in Subsubsection 3.1.2. Note that $M_{v}^{(i)}$ is a free $\Lambda(\kappa)^{(i)}$-module of rank two for every $i \in I_{v}$. Moreover we have

$$
\mathcal{F}\left(M_{v}^{(i)}\right) \subseteq M_{v}^{(i+1)} \quad \text { and } \quad \mathcal{V}\left(M_{v}^{(i)}\right) \subseteq M_{v}^{(i-1)}, \quad \forall(v, i)
$$

Let

$$
\underline{e}_{X}=\left(\underline{e}_{v}\right)_{v \in \Sigma_{E, p}}=\left(\left(e_{v, 1}^{(i)}, e_{v, 2}^{(i)}\right)_{i \in I_{v}}\right)_{v \in \Sigma_{E, p}}
$$

be the Lie type of $X$.

We will define an element $\underline{c}_{X_{v}}=\left(c_{X_{v}}^{(i)}\right)_{i \in I_{v}}$ in $\boldsymbol{\tau}_{E_{v}, \kappa} \stackrel{\sim}{\rightarrow} \boldsymbol{\tau}_{E_{v}, \kappa_{1}}$, called the congruity of the rank-two $\mathcal{O}_{E_{v}}$-linear $p$-divisible group $\left(X_{v}, \iota_{v}\right)$. For every $i \in I_{v}$, the $i$-component 
$c_{X_{v}}^{(i)}$ of $\underline{c}_{X_{v}}$ is given by

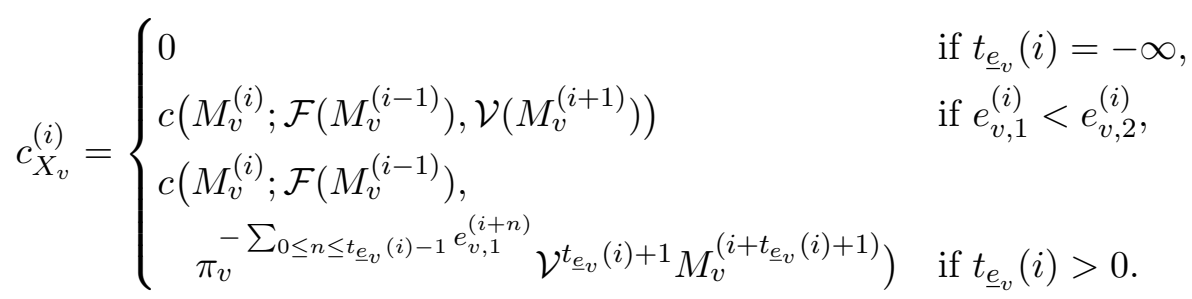

(b) Let $(X, \iota)$ be a rank-two $\mathcal{O}_{E}$-linear $p$-divisible group over $\kappa$. For every $v \in \Sigma_{E, p}$, let $X_{v}:=X\left[\wp_{v}^{\infty}\right]$, an $\mathcal{O}_{E_{v}}$-linear $p$-divisible group over $\kappa$. The congruity invariant $\underline{c}_{X}$ of $(X, \iota)$ is the element

$$
\underline{c}_{X}:=\left(\underline{c}_{X_{v}}\right)_{v \in \Sigma_{E, p}}=\left(\left(c_{X_{v}}^{(i)}\right)_{i \in I_{v}}\right)_{v \in \Sigma_{E, p}}
$$

of $\boldsymbol{\tau}_{E}=\boldsymbol{\tau}_{E, \kappa}$.

Remark 5.2.1. (a) In the case $t_{\underline{e}_{v}}(i)>0$, we have

$$
\left(\mathcal{V}\left(M_{v}\right)\right)^{(i+n)}=\mathcal{V}\left(M_{v}^{(i+n+1)}\right)=\pi_{v}^{e_{v, 1}^{(i+n)}} M_{v}^{(i+n)} \text { for } n=0,1, \ldots, t_{\underline{e}_{v}}(i)-1,
$$

and we use the $\sigma^{-1}$-linear isomorphisms

$$
\pi_{v}^{-e_{v, 1}^{(i+n)}} \mathcal{V}: M_{v}^{(i+n+1)} \stackrel{\sim}{\rightarrow} M_{v}^{(i+n)}, \quad n=0,1, \ldots, t_{\underline{e}_{v}}(i)-1
$$

to transport the lattice $\mathcal{V}\left(M_{v}^{\left(i+t_{\underline{e}_{v}}(i)+1\right)}\right)$ in $M_{v}^{\left(i+t_{\underline{e}_{v}}(i)\right)}$ back to $M_{v}^{(i)}$. The $(v, i)$-component of $\underline{c}_{X}$ is defined to be the congruity of $\mathcal{F}\left(M_{v}^{(i-1)}\right)$ and the result of the transport, two submodules of the free $\Lambda(\kappa)^{(i)}$-module $M_{v}^{(i)}$ of rank two.

(b) It is easy to see that the elementary divisors of the $\Lambda(\kappa)^{(i)}$-submodule $\mathcal{F}\left(M_{v}^{(i-1)}\right) \subseteq$ $M_{v}^{(i)}$ are $\left(e_{v}-e_{v, 2}^{(i-1)}, e_{v}-e_{v, 1}^{(i-1)}\right)$ because $\mathcal{V} \circ \mathcal{F}=p=\mathcal{F} \circ \mathcal{V}$. Therefore,

$$
0 \leq c_{X, v}^{(i)} \leq \min \left\{e_{v, 2}^{(i-1)}-e_{v, 1}^{(i-1)}, e_{v, 2}^{(i+t)}-e_{v, 1}^{(i+t)}\right\}, \quad \forall v \in \Sigma_{E, p}, \forall i \in I_{v},
$$

where $t=t_{\underline{e}_{v}}(i)$.

Remark 5.2.2. The congruity invariant $\underline{c}_{X}$ and the Lie type of $(X, \iota)$ determine the $\alpha$-type of $(X, \iota)$. The relations between the $\alpha$-type and the Newton polygon were investigated in $[39$.

Proposition 5.3. Let $v$ be a place of $E$ above $p$, and let $\left(X_{v}, \iota_{v}\right)$ be a rank-two $\mathcal{O}_{E_{v}}$-linear $p$-divisible group over an algebraically closed field $k \supseteq \mathbb{F}_{p}$. Let $\underline{e}_{X_{v}}=\left(e_{X_{v}, 1}^{(i)}, e_{X_{v}, 2}^{(i)}\right)_{i \in I_{v}}$, $\underline{c}_{X_{v}}=\left(c_{X_{v}}^{(i)}\right)_{i \in I_{v}}$ and $\underline{s}_{X_{v}}=\left(s_{X_{v}, 1}, s_{X_{v}, 2}\right)$ be the Lie type, congruity and Newton polygon of $\left(X_{v}, \iota_{v}\right)$ respectively. 
(1) $\left[E_{v}: \mathbb{Q}_{p}\right] \cdot \sum_{i \in I_{v}} e_{X_{v}, 1}^{(i)} \leq s_{X_{v}, 1}^{(i)}$.

(2) The following statements are equivalent.

(a) $\left[E_{v}: \mathbb{Q}_{p}\right] \cdot \sum_{i \in I_{v}} e_{X_{v}, 1}^{(i)}=s_{X_{v}, 1}^{(i)}$.

(b) $\underline{c}_{X_{v}}=\underline{0}$, i.e., $c_{X_{v}}^{(i)}=0$ for every $i \in I_{v}$.

(c) There exist

- isoclinic p-divisible groups $Y_{1}, Y_{2}$ over $k$ of height $\left[E_{v}: \mathbb{Q}_{p}\right]$ of slopes $s_{X_{v}, 1}$ and $s_{X_{v}, 2}$ respectively, and

- ring homomorphisms $\iota_{j}: \mathcal{O}_{E_{v}} \rightarrow \operatorname{End}\left(Y_{j}\right)$ for $j=1,2$, and

- an $\mathcal{O}_{E_{v}}$-linear isomorphism

$$
(X, \iota) \stackrel{\sim}{\rightarrow}\left(Y_{1}, \iota_{1}\right) \times\left(Y_{2}, \iota_{2}\right)
$$

See [9, Section 4.3] for a proof of Proposition 5.3 .

Remark 5.3.1. For $j=1,2$, denote by $\underline{e}_{Y_{j}}$ the Lie type of the CM $p$-divisible group $Y_{j}$ in Proposition 5.3 (2), i.e., the element of $\boldsymbol{\tau}_{E_{v}}$ corresponding to the $\left(\mathcal{O}_{E_{v}} \otimes_{\mathbb{Z}_{p}} k\right)$-module $\mathbb{D}\left(Y_{j}\right) / \mathcal{V}\left(\mathbb{D}\left(Y_{j}\right)\right)$. Then

$$
\underline{e}_{Y_{1}}=\left(e_{X_{v}, 1}^{(i)}\right)_{i \in I_{v}}, \quad \underline{e}_{Y_{2}}=\left(e_{X_{v}, 2}^{(i)}\right)_{i \in I_{v}}
$$

Since two $\mathcal{O}_{E_{v}}$-linear CM $p$-divisible groups over an algebraically closed field $k \supseteq \mathbb{F}_{p}$ with the same Lie type are $\mathcal{O}_{E_{v}}$-linearly isomorphic, the unordered pair $\left\{\left(Y_{1}, \iota_{1}\right),\left(Y_{2}, \iota_{2}\right)\right\}$ in Proposition 5.3 is uniquely determined by the congruity invariant $\underline{c}_{X_{v}}$ of $\left(X_{v}, \iota_{v}\right)$ up to isomorphism.

Definition 5.3.2 (Exquisite rank-two $\mathcal{O}_{E_{v}}$-linear $p$-divisible groups). Let $v$ be a place of $E$ above $p$ and let $\left(X_{v}, \iota_{v}\right)$ be a rank-two $\mathcal{O}_{E_{v}}$-linear $p$-divisible group over a field $K \supseteq \mathbb{F}_{p}$. We say that $\left(X_{v}, \iota_{v}\right)$ is exquisite if for some algebraically closed extension field $k$ of $K$, or equivalently for every algebraically closed extension field $k$ of $K$, the equivalent conditions (a)-(c) in Proposition 5.3(2) are satisfied.

Corollary 5.3.3. Let $v$ be a place of $E$ above $p$ and let $k \supseteq \mathbb{F}_{p}$ be an algebraically closed field. If $\left(X_{1}, \iota_{1}\right)$ and $\left(X_{2}, \iota_{2}\right)$ are two exquisite rank-two $\mathcal{O}_{E_{v}}$-linear $p$-divisible groups with the same Lie type, then there exists an $\mathcal{O}_{E_{v}}$-linear isomorphism from $\left(X_{1}, \iota_{1}\right)$ to $\left(X_{2}, \iota_{2}\right)$.

\section{4 .}

In this subsection we define the notion of balanced self-dual rank-two p-divisible groups. We begin with a preliminary statement in Lemma 5.4.1, whose proof is obvious. 
Lemma 5.4.1. Let $K$ be a field which contains a subfield isomorphic to $\kappa_{v}$, where $v$ is a place of $E$ above $p$. Let $\left(X_{v}, \iota_{v}\right)$ be a self-dual rank-two $\mathcal{O}_{E_{v}}$-linear $p$-divisible group over $K$ whose Lie type $\underline{e}_{X_{v}}$ is equal to the minimal element $\underline{e}_{E_{v}, \sin }^{\mathrm{sd}}$ in $\mathcal{T}_{E_{v}}^{\mathrm{sd}}$.

(a) Suppose that the ramification index $e_{v}$ of $E_{v} / \mathbb{Q}_{p}$ is even. Then the congruity $\underline{c}_{X_{v}}$ of $\left(X_{v}, \iota_{v}\right)$ is $\underline{0}$, i.e., $c_{X, v}^{(i)}=0$ for all $i \in I_{v}$.

(b) Suppose that the ramification index $e_{v}$ of $E_{v} / \mathbb{Q}_{p}$ is odd. Let $\underline{c}_{X_{v}}=\left(c_{X_{v}}^{(i)}\right)_{i \in I_{v}}$ be the congruity of $\left(X_{v}, \iota_{v}\right)$. Then $c_{X_{v}}^{(i)} \in\{0,1\}$ for every $i \in I_{v}$.

In the case of (b), the subset

$$
\left\{i \in I_{v} \mid c_{X_{v}}^{(i)}=1\right\}
$$

will be called the support of $\underline{c}_{X_{v}}$.

Definition 5.4.2 (Balanced subsets of $I_{v}$ ). (a) A non-empty subset $J$ of the $\left(\mathbb{Z} / f_{v} \mathbb{Z}\right)$ torsor $I_{v}$ is an interval if $J \varsubsetneqq I_{v}$ and there exists an element $i_{0} \in I$ and a natural number $b$ with $0 \leq b \leq f_{v}-2$ such that $J=\left\{i_{0}, i_{0}+1, \ldots, i_{0}+b\right\}$. Such an interval will be denoted by $\left[i_{0}, i_{0}+b\right]$.

(b) A subset $J$ of $I_{v}$ is connected if $J=\emptyset$, or if $J=I_{v}$, or if $J$ is an interval in $I_{v}$.

(c) A connected component of a subset $I^{\prime}$ of $I_{v}$ is a maximal element in the family of connected subsets of $I^{\prime}$.

(d) A subset $I^{\prime}$ of $I$ is balanced if the cardinality of every connected component of $I^{\prime}$ is even. In particular the empty subset $\emptyset$ is balanced, and $I_{v}$ is balanced if and only if $f_{v}$ is even.

Definition 5.4.3 (Balanced rank-two $\mathcal{O}_{E_{v}}$-linear $p$-divisible groups). Let $v$ be a place of $E$ above $p$. Let $K$ be a field which contains a finite subfield isomorphic to $\kappa_{v}$. A self-dual rank-two $\mathcal{O}_{E_{v}}$-linear $p$-divisible group $\left(X_{v}, \iota_{v}\right)$ over $K$ is said to be balanced if the following conditions are satisfied.

(a) The Lie type $\underline{e}_{X_{v}}$ of $\left(X_{v}, \iota_{v}\right)$ is equal to the minimal element

$$
\underline{e}_{E_{v}, \min }^{\mathrm{sd}}=\left(\left(\left\lfloor e_{v} / 2\right\rfloor,\left\lceil e_{v} / 2\right\rceil\right)\right)_{i \in I_{v}}
$$

in the local type space $\mathcal{T}_{E_{v}}^{\text {sd }}$.

(b) The support of $\underline{c}_{X_{v}}$ is a balanced subset of $I_{v}$.

Remark 5.4.4. Note that if the ramification index $e_{v}$ of $E_{v} / \mathbb{Q}_{p}$ is even, then the condition in Definition 5.4.3(b) is empty, and the congruity $\underline{c}_{X_{v}}$ of every self-dual rank-two $p$-divisible group $\left(X_{v}, \iota_{v}\right)$ over a field $K$ which satisfies Definition 5.4.3 (a) is both exquisite and balanced. So the notion of balanced self-dual rank-two $\mathcal{O}_{E_{v}}$-linear $p$-divisible groups is of interest only when $e_{v}$ is odd. 
Proposition 5.4.5. Let $v$ be a place of $E$ above $p$ such that $e_{v}=2 d_{v}+1$ is odd. Let $k \supseteq \mathbb{F}_{p}$ be an algebraically closed field.

(a) Let $\left(X_{v}, \iota_{v}\right)$ be a balanced rank-two $\mathcal{O}_{E_{v}}$-linear p-divisible group. Let $I^{\prime}$ be the support of $\underline{c}_{X_{v}} \neq \underline{0}$. Assume that $I^{\prime} \neq \emptyset$, and let $I_{1}$ be a subset of $I_{v}$ such that $I^{\prime}$ is the disjoint union of $I_{1}$ and $I_{1}+1$. There exist $\mathcal{O}_{E_{v}}$-linear $p$-divisible groups $\left(Y_{1}, \iota_{1}\right),\left(Y_{2}, \iota_{2}\right)$ over $k$ of height $\left[E_{v}: \mathbb{Q}_{p}\right]$ with the following properties:

- The Lie type $\underline{e}_{Y_{1}}=\left(e_{Y_{1}}^{(i)}\right)_{i \in I_{v}}$ of $\left(Y_{1}, \iota_{1}\right)$ is given by

$$
e_{Y_{1}}^{(i)}= \begin{cases}d_{v}+1 & \text { if } i \in I_{1} \\ d_{v} & \text { if } i \notin I_{1} .\end{cases}
$$

- The Lie type $\underline{e}_{Y_{2}}=\left(e_{Y_{2}}^{(i)}\right)_{i \in I_{v}}$ of $\left(Y_{2}, \iota_{2}\right)$ is given by

$$
e_{Y_{2}}^{(i)}= \begin{cases}d_{v} & \text { if } i \in I_{1} \\ d_{v}+1 & \text { if } i \notin I_{1} .\end{cases}
$$

- There exists an $\mathcal{O}_{E_{v}}$-linear isomorphism from $\left(X_{v}, \iota_{v}\right)$ to $\left(Y_{1}, \iota_{1}\right) \times\left(Y_{2}, \iota_{2}\right)$.

(b) Suppose that $\left(X_{v}, \iota_{v}\right)$ and $\left(X_{v}^{\prime}, \iota_{v}^{\prime}\right)$ are balanced rank-two $\mathcal{O}_{E_{v}}$-linear $p$-divisible groups over $k$ with the same congruity invariant. Then $\left(X_{v}, \iota_{v}\right)$ is $\mathcal{O}_{E_{v}}$-linearly isomorphic to $\left(X_{v}^{\prime}, \iota_{v}^{\prime}\right)$.

See [9, Section 4.4] for a proof of Proposition 5.4.5

Definition 5.5 ( $E$-minimal self-dual rank-two $\mathcal{O}_{E}$-linear $p$-divisible groups). Let $K$ be a field which contains a finite subfield with $p^{f_{E}}$ elements.

(a) Let $v$ place of $E$ above $p$, and let $\left(X_{v}, \iota_{v}\right)$ be a self-dual rank-two $\mathcal{O}_{E_{v}}$-linear $p$ divisible group over $K$. We say that $\left(X_{v}, \iota_{v}\right)$ is $E_{v}$-minimal if its Lie type $\underline{e}_{X_{v}}$ is the minimal element $\underline{e}_{E_{v}}^{\mathrm{sd}}$,min in $\mathcal{T}_{E_{v}}^{\mathrm{sd}}$, and its congruity $\underline{c}_{X_{v}}=\left(c_{X_{v}}^{(i)}\right)_{i \in I_{v}}$ is given by

$$
c_{X_{v}}^{(i)}= \begin{cases}0 & \text { if } e_{v} \text { is even } \\ 1 & \text { if } e_{v} \text { is odd }\end{cases}
$$

for all $i \in I_{v}$.

(b) Let $(X, \iota)$ be a self-dual rank-two $\mathcal{O}_{E}$-linear $p$-divisible group over $K$. We say that $(X, \iota)$ is E-minimal if the $v$-component $\left(X\left[\wp_{v}^{\infty}\right], \iota\left[\wp_{v}^{\infty}\right]\right)$ of $(X, \iota)$ is $E_{v}$-minimal for every place $v$ of $E$ above $p$. 
Remark 5.5.1. (a) If $\left(X_{v}, \iota_{v}\right)$ is an $E_{v}$-minimal self-dual rank-two $\mathcal{O}_{E_{v}}$-linear $p$-divisible group over an algebraically closed field $k \supseteq \mathbb{F}_{p}$, then the $p$-divisible group $X_{v}$ is superspecial in the sense that it is isomorphic to the product of $\left[E_{v}: \mathbb{Q}_{p}\right]$ copies of the $p$-divisible group attached to a supersingular elliptic curve over $k$.

(b) The terminology "E-minimal" comes from 31].

Lemma 5.5.2. Let $v$ be a place of $E$ above $p$, and let $k \supseteq \overline{\mathbb{F}_{p}}$ be an algebraically closed field. Any two $E_{v}$-minimal self-dual rank-two $\mathcal{O}_{E_{v}}$-linear -divisible groups are $\mathcal{O}_{E_{v}}$-linearly isomorphic.

Definition 5.6 (Distinguished self-dual rank-two $\mathcal{O}_{E}$-linear $p$-divisible groups). Let $K$ be a field which contains a finite subfield with $p^{f_{E}}$ elements. Let $(X, \iota)$ be a self-dual rank-two $\mathcal{O}_{E}$-linear $p$-divisible group over $K$. Write $(X, \iota)$ as a product of self-dual rank two $\mathcal{O}_{E_{v}}$-linear $p$-divisible groups over $K$ :

$$
(X, \iota)=\prod_{v \in \Sigma_{E, p}}\left(X_{v}, \iota_{v}\right),
$$

where $\left(X_{v}, \iota_{v}\right):=\left(X\left[\wp_{v}^{\infty}\right], \iota\left[\wp_{v}^{\infty}\right]\right)$ for each $v \in \Sigma_{E, p}$.

We say that $(X, \iota)$ is distinguished if for every $v \in \Sigma_{E, p}$, the self-dual rank-two $\mathcal{O}_{E_{v}}{ }^{-}$ linear $p$-divisible group $\left(X_{v}, \iota_{v}\right)$ is exquisite, or is balanced, or is $E_{v}$-minimal.

Proposition 5.6.1. Let $(X, \iota)$ and $\left(X^{\prime}, \iota^{\prime}\right)$ be distinguished self-dual rank-two $\mathcal{O}_{E}$-linear p-divisible groups over an algebraically closed field $k \supseteq \mathbb{F}_{p}$. If $(X, \iota)$ and $\left(X^{\prime}, \iota^{\prime}\right)$ have the same Lie type and the same congruity, then $(X, \iota)$ is $\mathcal{O}_{E}$-linearly isomorphic to $\left(X^{\prime}, \iota^{\prime}\right)$.

Proof. This is the combination of Corollary 5.3.3. Proposition 5.4.5, and Lemma 5.5.2.

\section{The stratification of a Lie stratum by congruity}

In this section $\mathscr{M}_{E}$ stands for a Hilbert modular variety $\mathscr{M}_{E, n, \bar{\delta}}^{\widetilde{\mathscr{L}}}$ over $\overline{\mathbb{F}_{p}}$, where $n \geq 3$ is a positive integer relatively prime to $p, \widetilde{\mathscr{L}}=\left(\mathscr{L}, \mathscr{L}_{\mathbb{R}}^{+}\right)$is an invertible $\mathcal{O}_{E^{-}}$-module with a notion of positivity, and $\bar{\delta}$ is an $\mathcal{O}_{E}$-generator of $\mathscr{L}^{\vee} \otimes \mathcal{O}_{E} \mathcal{D}_{E / \mathbb{Q}}^{-1} / n \mathscr{L}^{\vee} \otimes \mathcal{O}_{E} \mathcal{D}_{E / \mathbb{Q}}^{-1}$. We will stratify each Lie stratum $\mathcal{N}_{\underline{e}}$ of $\mathscr{M}_{E}$ by the congruity invariant, and explore properties of the resulting congruity stratification of $\mathcal{N}_{\underline{e}}$.

Definition 6.1 (Congruity strata in a Lie stratum $\mathcal{N}_{\underline{e}}$ ). Let

$$
\underline{e}=\left(\underline{e}_{v}\right)_{v \in \Sigma_{E, p}}=\left(\left(\left(e_{v, 1}^{(i)}, e_{v, 2}^{(i)}\right)\right)_{i \in I_{v}}\right)_{v \in \Sigma_{E, p}}
$$

be an element of $\mathcal{T}_{E}^{\mathrm{sd}}$, and let $\mathcal{N}_{\underline{e}}=\mathcal{N}_{\underline{e}}\left(\mathscr{M}_{E}\right)$ be the Lie stratum in $\mathscr{M}_{E}$ consisting of points corresponding to abelian varieties with real multiplication by $\mathcal{O}_{E}$ whose Lie type is equal to $\underline{e}$. 
For each $\underline{c} \in \boldsymbol{\tau}_{E}$, denote by $\mathcal{Q}_{\underline{c}}\left(\mathcal{N}_{\underline{e}}\right)$ the reduced locally closed subscheme of $\mathcal{N}_{\underline{e}}$ such that for every algebraically closed extension field $k$ of $\mathbb{F}_{p}$, the set $\mathcal{Q}_{\underline{c}}\left(\mathcal{N}_{\underline{e}}\right)(k)$ of $k$-points of $\mathcal{Q}_{\underline{c}}\left(\mathcal{N}_{\underline{e}}\right)$ consists of all points $x=\left[\left(A_{x}, \lambda_{x}, \iota_{x}, \eta_{x}\right)\right] \in \mathcal{N}_{\underline{e}}(k)$ such that the congruity invariant $\underline{c}_{x}$ of $\left(A_{x}, \iota_{x}\right)$ is equal to $\underline{c}$.

Clearly $\mathcal{N}_{\underline{e}}$ is the disjoint union of all congruity strata $\mathcal{Q}_{\underline{c}}\left(\mathcal{N}_{\underline{e}}\right)$, where $\underline{c}$ runs through all elements of $\boldsymbol{\tau}_{E}$. Note that there may exist pairs $(\underline{e}, \underline{c})$ such that $\mathcal{Q}_{c}\left(\mathcal{N}_{e}\right)$ is empty.

Proposition 6.2. (1) For every $\underline{c}$, the Zariski closure of $\mathcal{Q}_{\underline{c}}\left(\mathcal{N}_{\underline{e}}\right)$ is the disjoint union of all $\mathcal{Q}_{\underline{c}^{\prime}}\left(\mathcal{N}_{\underline{e}}\right)$ with $\underline{c}^{\prime} \preceq \underline{c}$ :

$$
\left(\mathcal{Q}_{\underline{c}}\left(\mathcal{N}_{\underline{e}}\right)\right)^{\mathrm{zar}}=\bigsqcup_{\underline{c^{\prime}} \preceq \underline{c}} \mathcal{Q}_{\underline{c}^{\prime}}\left(\mathcal{N}_{\underline{e}}\right) .
$$

In particular $\mathcal{Q}_{\underline{0}}\left(\mathcal{N}_{\underline{e}}\right)$ is a dense open subscheme of $\mathcal{N}_{\underline{e}}$.

(2) If $\mathcal{Q}_{c}\left(\mathcal{N}_{e}\right) \neq \emptyset$, then every irreducible component of $\mathcal{Q}_{c}\left(\mathcal{N}_{e}\right) \neq \emptyset$ is a locally closed smooth subscheme of $\mathcal{N}_{\underline{e}}$ of codimension $|\underline{c}|$ in $\mathcal{N}_{\underline{e}}$, where

$$
|\underline{c}|:=\sum_{v \in \Sigma_{E, p}} \sum_{i \in I_{v}} c_{v}^{(i)}
$$

(3) For each $\underline{c}$ with $|\underline{c}|=1$, the Zariski closure $\bigsqcup_{c^{\prime} \preceq c} \mathcal{Q}_{\underline{c}^{\prime}}\left(\mathcal{N}_{\underline{e}}\right)$ of $\mathcal{Q}_{\underline{c}}\left(\mathcal{N}_{\underline{e}}\right)$ is a smooth divisor on the smooth scheme $\mathcal{N}_{\underline{e}}$ over $\overline{\mathbb{F}_{p}}$. Together these smooth divisors on $\mathcal{N}_{\underline{e}}$ form a divisor with normal crossings, whose support is equal to $\mathcal{N}_{\underline{e}} \backslash \mathcal{Q}_{\underline{0}}\left(\mathcal{N}_{\underline{e}}\right)$.

Remark. Proposition 6.2 is proved using deformation theory of $p$-divisible groups and the theory of displays; see [9, Section 4.5].

Definition 6.2.1. A Lie-congruity stratum $\mathcal{Q}_{\underline{c}}\left(\mathcal{N}_{\underline{e}}\right)$ in $\mathscr{M}_{E}$ is said to be distinguished if for every $v \in \Sigma_{E, p}$, either $\underline{c}_{v}=\underline{0}$, or $\underline{e}_{v}=\underline{e}_{E_{v}}^{\text {sd } \text {,min }}$ and $\underline{c}_{v}$ is balanced.

Remark. It follows from Propositions 5.3 and 5.4 .5 that a Lie-congruity stratum $\mathcal{Q}_{\underline{c}}\left(\mathcal{N}_{\underline{e}}\right)$ is distinguished if and only if for every geometric point $x$ of $\mathcal{Q}_{\underline{c}}\left(\mathcal{N}_{\underline{e}}\right)$, the self-dual $\mathcal{O}_{E}$-linear $p$-divisible group attached to $x$ is distinguished.

\subsection{Central leaves in $\mathscr{M}_{E}$}

Through every $\overline{\mathbb{F}_{p}}$-point $x \in \mathscr{M}_{E}$, we have a smooth locally closed subscheme $\mathcal{C}(x)=$ $\mathcal{C}_{\mathscr{M}_{E}}(x) \subseteq \mathscr{M}_{E}$ satisfying the following properties.

(i) For every algebraically closed extension field $k$ of $\overline{\mathbb{F}_{p}}$, we have

$$
\mathcal{C}(x)(k)=\left\{y \in \mathscr{M}_{E}(x) \mid\left(A_{y}\left[p^{\infty}\right], \lambda_{y}\left[p^{\infty}\right], \iota_{y}\left[p^{\infty}\right]\right) \cong\left(A_{x}\left[p^{\infty}\right], \lambda_{x}\left[p^{\infty}\right], \iota_{x}\left[p^{\infty}\right]\right)\right\}
$$


(ii) The restriction to $\mathcal{C}(x)$ of the universal self-dual rank-two $\mathcal{O}_{E}$-linear principally $\widetilde{\mathscr{L}}$ polarized $p$-divisible group $(A, \lambda, \iota)\left[p^{\infty}\right]$ over $\mathcal{C}(x)$ is sustained, i.e., for every positive integer $m$, the $\mathcal{C}(x)$-scheme

$$
\boldsymbol{I}_{\boldsymbol{s o m}} \boldsymbol{m}_{(x)}\left(\left(A_{x}, \lambda_{x}, \iota_{x}\right)\left[p^{m}\right],(A, \lambda, \iota)\left[p^{m}\right]\right) \rightarrow \mathcal{C}(x)
$$

whose sections over every $\mathcal{C}(x)$-scheme $S$ is

$$
\operatorname{Isom}_{S}\left(\left(A_{x}, \lambda_{x}, \iota_{x}\right)\left[p^{m}\right] \times_{\operatorname{Spec}(k)} S,(A, \lambda, \iota)\left[p^{m}\right] \times_{\mathcal{C}(x)} S\right),
$$

is faithfully flat over $\mathcal{C}(x)$. See [9, Chapter 5] for the theory of sustained $p$-divisible groups.

(iii) For every positive integer $m$, there exist an integer $j_{0}$ such that the images of the restriction morphisms

$$
\begin{aligned}
& \boldsymbol{I s o m}_{C(x)}\left(\left(A_{x}, \lambda_{x}, \iota_{x}\right)\left[p^{m+j}\right],(A, \lambda, \iota)\left[p^{m+j}\right]\right) \\
\rightarrow & \boldsymbol{I s o m}_{C(x)}\left(\left(A_{x}, \lambda_{x}, \iota_{x}\right)\left[p^{m}\right],(A, \lambda, \iota)\left[p^{m}\right]\right)
\end{aligned}
$$

stabilize for all $j \geq j_{0}$ and defines a subscheme

$$
\boldsymbol{I}_{\boldsymbol{s o m}}^{\mathrm{stab}}\left(\left(A_{x}, \lambda_{x}, \iota_{x}\right)\left[p^{m}\right],(A, \lambda, \iota)\left[p^{m}\right]\right)
$$

of $\boldsymbol{I s o m}_{C(x)}\left(\left(A_{x}, \lambda_{x}, \iota_{x}\right)\left[p^{m}\right],(A, \lambda, \iota)\left[p^{m}\right]\right)$, called the stabilized Isom scheme for $\mathcal{C}(x)$ at level $m$, which is finite locally free over $\mathcal{C}(x)$.

Remark 6.3.1. (a) The condition (i) uniquely determines $\mathcal{C}(x)$ as a subset of $\mathscr{M}_{E}$. The path adopted in [32], which may be called the "direct approach", is to first show that this subset is constructible. Then one shows, in a bootstrapping process, that the subset $\mathcal{C}(x)$ with reduced scheme structure is locally closed and smooth.

(b) The approach to central leaves in [9] via the notion of sustained $p$-divisible groups explained in [9, Chapter 5] has the advantage of conceptual clarity and helps revealing the local structures of central leaves. See Proposition 6.3.3 for an illustration.

Lemma 6.3.2. Let $x$ be an $\overline{\mathbb{F}_{p}}$-point of $\mathscr{M}_{E}$, let $\mathcal{C}(x)$ be the central leaf in $\mathscr{M}_{E}$ containing $x$, and let $\underline{s}=\left(\left(s_{v, 1}, s_{v, 2}\right)\right)_{v \in \Sigma_{E, p}} \in \operatorname{NP}_{E}^{\text {sd }}$ be the Newton polygon for $\left(A_{x}, \iota_{x}\right)$. Then

$$
\operatorname{dim}(\mathcal{C}(x))=\sum_{v \in \Sigma_{E, p}}\left[E_{v}: \mathbb{Q}_{p}\right] \cdot\left(s_{v, 2}-s_{v, 1}\right)
$$

Proposition 6.3.3. For every $\overline{\mathbb{F}_{p}}$-point $x$ of $\mathscr{M}_{x}$, the leaf $\mathcal{C}_{\mathscr{M}_{E}}(x)$ in $\mathscr{M}_{E}$ is quasi-affine. 
Proof. Consider the stabilized Isom scheme

$$
\boldsymbol{I}_{\boldsymbol{s o m}}^{\mathrm{stab}}=\boldsymbol{I} \boldsymbol{s o m}_{C(x)}^{\mathrm{stab}}\left(\left(A_{x}, \lambda_{x}, \iota_{x}\right)\left[p^{m}\right],(A, \lambda, \iota)\left[p^{m}\right]\right)
$$

for $\mathcal{C}(x)$ at level $m$, which is finite locally free over $\mathcal{C}(x)$. The pullback to $\boldsymbol{I s o m}_{m}^{\text {stab }}$ of the restriction to $\mathcal{C}(x)$ of the universal $p$-divisible group is constant. Therefore the pullback to $\boldsymbol{I s o m}_{m}^{\text {stab }}$ of the Hodge line bundle on $\mathscr{M}_{E}$ is trivial. As the Hodge bundle on $\mathscr{M}_{E}$ is ample, the proposition follows.

Proposition 6.3.4. A distinguished congruity stratum $\mathcal{Q}_{\underline{c}}\left(\mathcal{N}_{\underline{e}}\right)$ in a Lie stratum $\mathcal{N}_{\underline{e}}$ is a central leaf.

Proof. This is a rehash of Proposition 5.6.1.

Definition 6.3.5. A central leaf in $\mathscr{M}_{E}$ which is equal to a distinguished congruity stratum in a Lie stratum $\mathcal{N}_{\underline{e}}$ is called a distinguished central leaf in $\mathscr{M}_{E}$; cf. Definition 6.2.1.

Lemma 6.3.6. Newton polygons of distinguished central leaves in $\mathscr{M}_{E}$ exhausts all Newton polygons for $E$. In other words for every $\underline{s} \in \mathrm{NP}_{E}^{\mathrm{sd}}$, there exists a distinguished central leaf in $\mathscr{M}_{E}$ whose Newton polygon is $\underline{s}$.

Proposition 6.4 below, on the incidence relation between distinguished congruity strata and the $E$-minimal locus follows from deformation theoretic considerations in 13 , Sections 3-4] and the proof of Proposition 6.2. cf. [9, Section 4.5]. It is an important ingredient in the proof that prime-to- $p$ Hecke correspondences operate transitively on the set of irreducible components of a distinguished central leaf in $\mathscr{M}_{E}$.

Proposition 6.4. Let $\mathcal{Q}_{\underline{c}}\left(\mathcal{N}_{\underline{e}}\right)$ be a distinguished Lie-congruity stratum on $\mathscr{M}_{E}$. Let $\mathcal{Q}^{\prime}$ and $\mathcal{Q}^{\prime \prime}$ be irreducible components of $\mathcal{Q}_{\underline{c}}\left(\mathcal{N}_{\underline{e}}\right)$. If there is an E-minimal point $z \in \mathcal{N}_{\text {min }}$ which is contained in the Zariski closure of $\mathcal{Q}^{\prime}$ and also the Zariski closure of $\mathcal{Q}^{\prime \prime}$, then $\mathcal{Q}^{\prime}=\mathcal{Q}^{\prime \prime}$.

7. Distinguished leaves on non-minimal Lie strata

Theorem 7.1. Let $\mathcal{N}_{\underline{e}}$ be a non-minimal Lie stratum in $\mathscr{M}_{E}$, i.e., $\underline{e} \neq \underline{e}_{E, \sin }^{\mathrm{sd}}$. No irreducible component of a distinguished Lie-congruity stratum $\mathcal{Q}_{\underline{c}}\left(\mathcal{N}_{\underline{e}}\right)$ is closed in $\mathscr{M}_{E}$. More precisely, for every distinguished Lie-congruity stratum $\mathcal{Q}_{\underline{c}}\left(\mathcal{N}_{\underline{e}}\right)$ on $\mathcal{N}_{\underline{e}}$, the Zariski closure in $\mathscr{M}_{E}$ of every irreducible component of $\mathcal{Q}_{\underline{c}}\left(\mathcal{N}_{\underline{e}}\right)$ contains an irreducible component of the distinguished Lie-congruity stratum $\mathcal{Q}_{\underline{c}}\left(\mathcal{N}_{\text {min }}\right)$ of the minimal Lie stratum $\mathcal{N}_{\text {min }}$ in $\mathscr{M}_{E}$.

Theorem 7.2 below is a slightly stronger version of Theorem 7.1 , with the same proof. 
Theorem 7.2. Let $\underline{e}=\left(\underline{e}_{v}\right)_{v \in \Sigma_{E, p}}$ be an element of $\mathcal{T}_{E}^{\mathrm{sd}}$ and let $\underline{c}=\left(\underline{c}_{v}\right)_{v \in \Sigma_{E, p}}$ be an element of $\boldsymbol{\tau}_{E}$. Let $\mathcal{Q}$ be an irreducible component of a Lie-congruity stratum $\mathcal{Q}_{\underline{c}}\left(\mathcal{N}_{\underline{e}}\right)$ in a Lie stratum $\mathcal{N}_{\underline{e}}$ of $\mathscr{M}_{E}$. Suppose that $v_{0}$ is a place of $E$ above $p$ such that $\underline{e}_{v_{0}} \neq$ $\underline{e}_{E_{v_{0}}}^{\mathrm{sd} \text {,min }}$ and $\underline{c}_{v_{0}}=\underline{0}$. In other words the self-dual rank-two $\mathcal{O}_{E_{v_{0}}}$-linear $p$-divisible group $\left(A_{x}\left[\wp_{v_{0}}^{\infty}\right], \iota_{x}\left[\wp_{v_{0}}^{\infty}\right]\right)$ attached to any geometric point $x$ of $\mathcal{Q}_{\underline{c}}\left(\mathcal{N}_{\underline{e}}\right)$ is distinguished and its Lie type is not equal to the minimal element $\underline{e}_{E_{v} \text {,min }}^{\mathrm{sd}}$ in $\mathcal{T}_{E_{v}}^{\mathrm{sd}}$. Then the Zariski closure $\mathcal{Q}^{\mathrm{zar}}$ of $\mathcal{Q}$ contains an irreducible component of $\mathcal{Q}_{\underline{c}}\left(\mathcal{N}_{\underline{e}^{\prime}}\right)$, where $\underline{e}^{\prime}=\left(\underline{e}_{v}^{\prime}\right)_{v \in \Sigma_{E, p}}$ is the element of $\mathcal{T}_{E}^{\text {sd }}$ given by

$$
\underline{e}_{v}^{\prime}= \begin{cases}\underline{e}_{E_{v_{0}}, \min }^{\mathrm{sd}} & \text { if } v=v_{0}, \\ \underline{e}_{v} & \text { if } v \neq v_{0} .\end{cases}
$$

Remark 7.3. The reason that we need a statement like Theorem 7.1 is that a priori, it is possible that a given distinguished Lie-congruity stratum $\mathcal{Q}_{\underline{c}}\left(\mathcal{N}_{\underline{e}}\right)$ with $\mathcal{N}_{\underline{e}} \neq \mathcal{N}_{\text {min }}$ may have an irreducible component $\mathcal{Q}$ which is equal to its own Zariski closure in $\mathscr{M}_{E}$. In the case when $\underline{c}$ is the maximal element $\underline{0}$ in $\tau_{E}$ and $\underline{e}$ is not the minimal element $\underline{e}_{E, \sin }^{\mathrm{sd}}$ in $\mathcal{T}_{E}^{\text {sd }}$, the scenario that $\mathcal{Q}$ is closed in $\mathscr{M}_{E}$ is equivalent to the statement that $\mathcal{Q}$ is proper over $\overline{\mathbb{F}_{p}}$. Most people will consider this scenario to be very unlikely, but we don't know of any "easy proof".

Readers who are contemplating going through the details of this proof in [9, Chapter 4] undoubtedly will prefer a proof which is shorter and more appealing. Unfortunately such a proof has yet to be found.

\section{4 .}

The main thrust of the proof of Theorem 7.1, described in [40, 2.5] and [9, Chapter 4], is an inductive procedure to construct, for any given irreducible component $\mathcal{Q}$ of a distinguished Lie-congruity stratum $\mathcal{Q}_{\underline{c}}\left(\mathcal{N}_{\underline{e}}\right)$ with $\mathcal{N}_{\underline{e}} \neq \mathcal{N}_{\text {min }}, \mathbb{P}^{1}$-families in the Zariski closure of $\mathcal{Q}$. Each of these $\mathbb{P}^{1}$ families of abelian varieties with real multiplication by $\mathcal{O}_{E}$ is $\mathcal{O}_{E}$-linearly isogenous to a constant abelian variety with real multiplication by $\mathcal{O}_{E}$, and there is a dense open subset $U$ of $\mathbb{P}^{1}$ contained in $\mathcal{Q}_{\underline{c}}\left(\mathcal{N}_{\underline{e}}\right)$. The Lie types of points of $\mathbb{P}^{1} \backslash U$ can be explicitly computed. In the majority of situations there is a point in $\mathbb{P}^{1} \backslash U$ whose Lie type is strictly small than $\underline{e}$ in $\mathcal{T}_{e}^{\text {sd }}$, and the inductive procedure stops if this is the case. If the Lie types of points of $\mathbb{P}^{1} \backslash U$ are all equal to $\underline{e}$, one constructs another $\mathbb{P}^{1}$ family and then repeats the same operation. One shows that this procedure eventually produces a point of the minimal Lie stratum $\mathcal{N}_{\underline{e}}$ which lies in the Zariski closure of $\mathcal{Q}$. 


\section{Congruity strata on the minimal Lie stratum}

Theorem 8.1. Every irreducible component of a congruity stratum of the minimal Lie stratum $\mathcal{N}_{\text {min }}=\mathcal{N}_{\underline{e}_{E, \text { min }}^{\text {sd }}}$ of $\mathscr{M}_{E}$ contains a minimal point, i.e., an irreducible component of the zero-dimensional congruity stratum in $\mathcal{N}_{\min }$.

\section{2 .}

The proof of Theorem 8.1 is a variant of the method called Raynaud's trick in [28, Section 4], and is based on the phenomenon, explained in Subsubsection 8.2.2, that the congruity stratification of the minimal Lie stratum $\mathcal{N}_{\min }\left(\mathscr{M}_{E}\right)$ bears prominent similarities to the $\alpha$-type stratification of Hilbert modular varieties attached to a totally real field $L$ which is unramified above $p$. See [9, Section 4.7] for details.

8.2.1. For a totally real field $L$ unramified above $p$, there is only one Lie stratum, and the congruity invariant on $\mathscr{M}_{L}$ has the same information content as the type of the maximal $\alpha$-subgroup schemes considered in [14]. It is shown in [14, Section 3] that for every $\alpha$-type stratum $W_{\tau}$ of $\mathscr{M}_{L}$, there exists a positive integer $m>0$ such that the restriction to $W_{\tau}$ of the $m$-th power of the Hodge line bundle on $\mathscr{M}_{L}$ is trivial, hence every $\alpha$-stratum $W_{\tau}$ of $\mathscr{M}_{L}$ is quasi-affine.

8.2.2. Let $x \in \mathcal{N}_{\min }\left(\overline{\mathbb{F}_{p}}\right)$ be an $\overline{\mathbb{F}_{p}}$-point of the minimal Lie stratum of $\mathscr{M}_{E}$. Let $M_{v}=$ $\mathbb{D}\left(A_{x}\left[\wp_{v}^{\infty}\right]\right)$ be the Dieudonné module of the $\mathcal{O}_{E_{v}}$-linear $p$-divisible group $A_{x}\left[\wp_{v}^{\infty}\right]$. The assumption that $x \in \mathcal{N}_{\min }\left(\overline{\mathbb{F}_{p}}\right)$ implies that the operators $\mathcal{F}, \mathcal{V}$ on $M_{v}$ are both divisible by $\pi_{v}^{\left\lfloor e_{v} / 2\right\rfloor}$. Let $\Phi_{v}:=\left.\pi_{v}^{-\left\lfloor e_{v} / 2\right\rfloor} \cdot \mathcal{F}\right|_{M_{v}}, \Psi_{v}:=\left.\pi_{v}^{-\left\lfloor e_{v} / 2\right\rfloor} \cdot \mathcal{V}\right|_{M_{v}}$. These two semi-linear operators on $M_{v}$ commute with the action of $\mathcal{O}_{v} \otimes_{\mathbb{Z}_{p}} \Lambda\left(\overline{\mathbb{F}_{p}}\right)$, and

$$
\Phi_{v} \circ \Psi_{v}=\Psi_{v} \circ \Phi_{v}=p \cdot \pi_{v}^{-2\left\lfloor e_{v} / 2\right\rfloor} .
$$

(i) If $e_{v}$ is even, then $\Phi_{v}$ is a $\sigma$-linear automorphism of $M_{v}, \Psi_{v}$ is a $\sigma^{-1}$-linear automorphism, and such a triple $\left(M_{v}, \Phi_{v}, \Psi_{v}\right)$ is rigid.

(ii) On the other hand if $e_{v}$ is odd, then $\pi_{v}^{\prime}:=p \cdot \pi_{v}^{-2\left\lfloor e_{v} / 2\right\rfloor}$ is a generator of $\wp_{v}$. For the decomposition $M_{v}=\bigoplus_{i \in I_{E_{v}}} M_{v}^{(i)}$ and the semi-linear operators $\Phi_{v}, \Psi_{v}$, we have

$$
\Phi_{v}\left(M_{v}^{(i)}\right) \subseteq M_{v}^{(i+1)}, \quad \Psi_{v}\left(M^{(i)}\right) \subseteq M_{v}^{(i-1)}, \quad \forall i \in I_{E_{v}}
$$

It follows that $\operatorname{dim}_{\overline{\mathbb{F}_{p}}}\left(M_{v}^{(i)} / \Phi_{v}\left(M_{v}^{(i-1)}\right)+\Psi_{v}\left(M_{v}^{(i+1)}\right)\right) \leq 1$ for every $i \in I_{E_{v}}$. The congruity $\underline{c}_{x, v}=\left(c_{x, v}^{(i)}\right)_{i \in I_{E_{v}}}$ of $A_{x}\left[\wp_{v}^{\infty}\right]$ is given by

$$
c_{x, v}^{(i)}= \begin{cases}0 & \text { if } \Phi_{v}\left(M_{v}^{(i-1)}\right)+\Psi_{v}\left(M_{v}^{(i+1)}\right)=M_{v}^{(i)}, \\ 1 & \text { if } \Phi_{v}\left(M_{v}^{(i-1)}\right)+\Psi_{v}\left(M_{v}^{(i+1)}\right) \neq M_{v}^{(i)} .\end{cases}
$$


This formula for $c_{x, v}^{(i)}$ is very similar to the formula for the $(w, i)$-component of the $\underline{a}$-invariant of an abelian variety with real multiplication by the ring of integers of a totally real field $L$ unramified above $p$. Of course if $E_{v}$ is unramified over $\mathbb{Q}_{p}$, then $e_{v}=1$, and $c_{x, v}^{(i)}=1$ if and only if $\mathcal{F}\left(M_{v}^{(i-1)}\right)+\mathcal{V}\left(M_{v}^{(i+1)}\right) \varsubsetneqq M_{v}^{(i)}$.

8.2.3. The similarity of the congruity stratification of the minimal Lie stratum $\mathcal{N}_{\underline{e}}$ on $\mathscr{M}_{E}$ with the $\alpha$-type stratification of $\mathscr{M}_{L}$, as sketched in Subsubsectin 8.2.2, holds to such an extent that the argument in [14, Section 3], suitably interpreted, shows that the restriction of the Hodge line bundle on $\mathscr{M}_{E}$ to every congruity stratum $\mathcal{Q}_{\underline{c}}\left(\mathcal{N}_{\text {min }}\right)$ on $\mathcal{N}_{\text {min }}$ is torsion. Therefore every congruity stratum $\mathcal{Q}_{\underline{c}}\left(\mathcal{N}_{\text {min }}\right)$ on $\mathcal{N}_{\text {min }}$ is quasi-affine.

\section{Irreducibility of non-supersingular distinguished leaves}

Theorem 9.1. The Zariski closure of every irreducible component of a distinguished Liecongruity stratum in $\mathscr{M}_{E}$ contains an E-minimal point.

Proof. Theorem 9.1 follows from Theorems 7.1 and 8.1 .

Replacing Theorem 7.1 by Theorem 7.2 , we get a slightly stronger statement.

Theorem 9.2. Let $\mathcal{Q}_{\underline{c}}\left(\mathcal{N}_{\underline{e}}\right)$ be a Lie-congruity stratum in $\mathscr{M}_{E}$. Suppose that for every place $v$ of $E$ above $p$, either the $v$-component $\underline{e}_{v}$ of $\underline{e}$ is equal to $\underline{e}_{E_{v}}^{\mathrm{sd}}$, min , or the $v$-component $\underline{c}_{v}$ of $\underline{c}$ is $\underline{0}$. Then every irreducible component of $\mathcal{Q}_{\underline{c}}\left(\mathcal{N}_{\underline{e}}\right)$ contains an E-minimal point.

Corollary 9.3. The prime-to-p Hecke correspondences operate transitively on the set of all irreducible components of every non-supersingular distinguished Lie-congruity stratum of $\mathscr{M}_{E}$.

Proof. This is a corollary of Theorem 9.1 and Proposition 6.4.

Corollary 9.4. Every non-supersingular distinguished Lie-congruity stratum in $\mathscr{M}_{E}$ is irreducible.

Proof. This irreducibility statement is a consequence of Corollary 9.3 and the method in 6 , 4.4] for proving irreducibility of Hecke-invariant subvarieties via prime-to- $p$ monodromy. Note that [6,4.4] was stated for Siegel modular varieties, but the proof therein shows that the statement also holds for Hilbert modular varieties; see [42, Section 6].

The same argument, using Theorem 9.2 instead of Theorem 9.1, gives a strengthened version of Corollary 9.4 . 
Corollary 9.5. Let $\mathcal{Q}_{\underline{c}}\left(\mathcal{N}_{\underline{e}}\right)$ be a Lie-congruity stratum in $\mathscr{M}_{E}$ which is not contained in the supersingular locus of $\mathscr{M}_{E}$. Suppose that for every place $v$ of $E$ above $p$, either the $v$-component $\underline{e}_{v}$ of $\underline{e}$ is equal to $\underline{e}_{E_{v}, \sin }^{\mathrm{sd}}$, or the $v$-component $\underline{c}_{v}$ of $\underline{c}$ is $\underline{0}$. Then $\mathcal{Q}_{\underline{c}}\left(\mathcal{N}_{\underline{e}}\right)$ is irreducible.

Remark 9.6. Readers may consult [9, Chapter 8] for a proof of the Hecke orbit conjecture for Siegel modular varieties, and see Corollary 9.4 in action. See also Subsection 1.1 for the role played by the Hilbert trick and the irreducibility result in Corollary 9.4 in the proof of the Hecke orbit conjecture.

10. Some questions

Question 10.1 (Geometry of the Lie-congruity strata). (a) Determine the Zariski closure $\left(\mathcal{Q}_{\underline{c}}\left(\mathcal{N}_{\underline{e}}\right)\right)^{\text {zar }}$ of the Lie-congruity strata $\mathcal{Q}_{\underline{c}}\left(\mathcal{N}_{\underline{e}}\right)$ in $\mathscr{M}_{E}$, for all $\underline{e} \in \mathcal{T}_{E}^{\text {sd }}$ and all $\underline{c} \in \boldsymbol{\tau}_{E}$.

(b) Is $\left(\mathcal{Q}_{\underline{c}}\left(\mathcal{N}_{\underline{e}}\right)\right)^{\text {zar }}$ normal, Cohen-Macaulay, or a local complete intersection?

(c) Is every Lie-congruity stratum $\mathcal{Q}_{c}\left(\mathcal{N}_{e}\right)$ quasi-affine?

Remark 10.1.1. An optimist may ask whether there exists a stratification on $\mathscr{M}_{E}$ which refines the Lie stratification on $\mathscr{M}_{E}$ and induces the congruity stratification on every Lie stratum $\mathcal{N}_{\underline{e}}$. In other words:

Is the Zariski closure of a Lie-congruity stratum $\mathcal{Q}_{\underline{c}}\left(\mathcal{N}_{\underline{e}}\right)$ a union of Lie-congruity strata, for every pair $(\underline{e}, \underline{c}) \in \mathcal{T}_{E}^{\mathrm{sd}} \times \boldsymbol{\tau}_{E}$ ?

Remark 10.1.2. Question 10.1 contains a preliminary part, namely determine all pairs $(\underline{e}, \underline{c}) \in \mathcal{T}_{E}^{\text {sd }} \times \boldsymbol{\tau}_{E}$ such that $\mathcal{Q}_{\underline{c}}\left(\mathcal{N}_{\underline{e}}\right) \neq \emptyset$. The related problem on non-emptiness of EO strata and Newton strata of PEL-type Shimura varieties has been solved in [36].

Remark 10.1.3. We know that every distinguished Lie-congruity stratum is quasi-affine, and so is every congruity stratum on the minimal Lie stratum $\mathcal{N}_{\text {min }}$. An optimist may ask whether the restriction of the Hodge line bundle to $\mathcal{Q}_{\underline{c}}\left(\mathcal{N}_{\underline{e}}\right)$ is torsion for every pair $(\underline{e}, \underline{c})$ in $\mathcal{T}_{E}^{\text {sd }} \times \tau_{E}$.

Question 10.2 (Irreducibility question for Lie-congruity strata). Let $\mathcal{Q}_{\underline{c}}\left(\mathcal{N}_{\underline{e}}\right)$ be a Liecongruity stratum not contained in the supersingular locus of $\mathscr{M}_{E}$. Is $\mathcal{Q}_{\underline{c}}\left(\mathcal{N}_{\underline{e}}\right)$ irreducible?

Question 10.3 (Lie-congruity strata and the Newton polygon stratification).

(a) Determine the subset

$$
\left\{(\underline{s}, \underline{e}, \underline{c}) \in \mathrm{NP}_{E}^{\mathrm{sd}} \times \mathcal{T}_{E}^{\mathrm{sd}} \times \tau_{E} \mid \mathcal{W}_{\underline{s}} \cap \underline{Q}_{\underline{c}}\left(\mathcal{N}_{\underline{e}}\right) \neq \emptyset\right\}
$$

of $\mathrm{NP}_{E}^{\mathrm{sd}} \times \mathcal{T}_{E}^{\mathrm{sd}} \times \boldsymbol{\tau}_{E}$. 
(b) Given a Lie-congruity stratum $\mathcal{Q}_{\underline{c}}\left(\mathcal{N}_{\underline{e}}\right)$, determine the Newton polygon of the generic point of each irreducible component of $\mathcal{Q}_{\underline{c}}\left(\mathcal{N}_{\underline{e}}\right)$.

(c) Given a Newton polygon stratum $\mathcal{W}_{\underline{s}}$, determine the Lie type and the congruity of the generic point of each irreducible component of $\mathcal{W}_{\underline{s}}$.

Question 10.4 (Newton polygon strata and Grothendieck's conjecture).

(a) For each Newton polygon $\underline{s} \in \mathrm{NP}_{E}^{\text {sd }}$, determine the number and dimensions of irreducible components of $\mathcal{W}_{\underline{s}}$. Compare the set of irreducible components of $\mathcal{W}_{\underline{s}}$ and that of the special fiber the Rapoport-Zink space attached to $\mathcal{W}_{\underline{s}}$ modulo the twisted stabilizer group (denoted by $J\left(\mathbb{Q}_{p}\right)$ in $[34$, p. xiii]).

(b) Determine the singular locus of the Zariski closure $\left(\mathcal{W}_{\underline{s}}\right)^{\text {zar }}$ of $\mathcal{W}_{\underline{s}}$. Is every irreducible component of $\left(\mathcal{W}_{\underline{s}}\right)^{\text {zar }}$ normal (respectively Cohen-Macaulay)?

(c) Let $k \supseteq \mathbb{F}_{p}$ be an algebraically closed field. Let $\underline{e}=\left(\left(e_{v, 1}^{(i)}, e_{v, 2}^{(i)}\right)_{i \in I_{v}}\right)_{v \in \Sigma_{E, p}}$ be an element of $\mathcal{T}_{E}^{\text {sd }}$. Let $x$ be a $k$-point of the Lie stratum $\mathcal{N}_{\underline{e}}$ in $\mathscr{M}_{E}$. Let $\underline{s}_{x}$ be the Newton polygon of $x$. Let $\underline{s}=\left(\left(s_{v, 1}, s_{v, 2}\right)\right) \in \mathrm{NP}_{E}^{\text {sd }}$ be a Newton polygon such that $\underline{s}_{x} \preceq \underline{s}$ and $s_{v, 1} \cdot\left[E_{v}: \mathbb{Q}_{p}\right] \geq \sum_{i \in I_{v}} e_{v, 1}$ for all $v \in \Sigma_{E, p}$. Show that there exists a point $y$ of $\mathcal{N}_{\underline{e}}$ such that $x$ is contained in the Zariski closure of $y$ and the Newton polygon of $y$ is equal to $\underline{s}$.

(d) Let $k \supseteq \mathbb{F}_{p}$ be an algebraically closed field. Let $x$ be a $k$-point of the Lie stratum $\mathcal{N}_{\underline{e}}$ in $\mathscr{M}_{E}$. Let $\underline{s}_{x}$ be the Newton polygon of $x$. Let $\underline{s}=\left(\left(s_{v, 1}, s_{v, 2}\right)\right) \in \mathrm{NP}_{E}^{\text {sd }}$ be a Newton polygon such that $\underline{s}_{x} \preceq \underline{s}$. Show that there exists a point $y$ of $\mathscr{M}_{E}$ whose Newton polygon is equal to $\underline{s}$ and $x$ is contained in the Zariski closure of $y$.

Remark. (i) It is not difficult to see that the statement (c) implies the statement (d). So the question (c) is in essence an approach to (d), which is Grothendieck's conjecture on the Newton polygon stratification for Hilbert modular varieties.

This conjecture of Grothendieck is known when $p$ is unramified in $E$ [18, 19], and also when every prime ideal of $\mathcal{O}_{E}$ containing $p \mathcal{O}_{E}$ has residue field $\mathbb{F}_{p}[38$, Theorem 6.20].

(ii) For the question (a) when $p$ is unramified in $E$ and the residue field degree $f_{v} \leq 4$ for every place $v \in \Sigma_{E, p}$, the number of the irreducible components of the supersingular locus is computed by a formula [41, Theorem 4.12]; this formula involves the special value $\zeta_{E}(-1)$ of the Dedekind zeta function of $E$ and certain constants $c(v)$ depending only on $f_{v}, v \in \Sigma_{E, p}$. Conjecturally [41, 4.13] the same formula for the number of irreducible components of the supersingular locus in $\mathscr{M}_{E}$ holds for all totally real field $E$, with the integer-valued constants $c(v)$ depending only on $e_{v}$ and $f_{v}, v \in \Sigma_{E, p}$. 
(iii) The sets of irreducible components of affine Deligne-Lusztig varieties modulo the action of the twisted stabilizer group are studied by Hamacher and Viehmann [20] and by Xiao and Zhu [37] very recently under a general good reduction setting. Their results give an explicit group-theoretic description of irreducible components of the corresponding Rapoport-Zink space in the question (a) when $p$ is unramified in $E$.

(iv) For a general formulation of the notion of Newton invariant of isocrystals with additional structure, see 22,24$]$.

Question 10.5 (Lie-congruity vs. EKOR strata). Compare the Lie-congruity stratification of $\mathscr{M}_{E}$ with the EKOR stratification in [21].

Remark. The Lie stratification on $\mathscr{M}_{E}$ coincides with the KR stratification introduced in 25, 27]. The Lie-congruity stratification and the EKOR stratification both refine the Lie stratification on $\mathscr{M}_{E}$. The geometry of KR strata of Siegel modular varieties is studied in 15 17.

Question 10.6 (Analog of the EO stratification on $\mathscr{M}_{E}$ ). Find a good definition of the Ekedahl-Oort stratification for Hilbert modular varieties, so that the resulting EO stratification on $\mathscr{M}_{E}$ has properties similar to the EO stratification [29] on the Siegel moduli space $\mathscr{A}_{g, 1, n} g$-dimensional principally polarized abelian varieties over $\overline{\mathbb{F}_{p}}$ with symplectic level- $n$ structures.

Remark 10.6.1. The EO stratification on $\mathscr{A}_{g, 1, n}$ comes from geometric isomorphism classes of $(A[p], \lambda[p])$, where $(A, \lambda)$ runs through $g$-dimensional principally polarized abelian varieties in characteristic $p$. The naive generalization to $\mathscr{M}_{E, n, \bar{\delta}}^{\widetilde{\mathscr{L}}}$ involves understanding the classification of geometric isomorphism classes of triples $(A[p], \lambda[p], \iota[p])$, where $[(A, \lambda, \iota, \eta)]$ runs through geometric points of $\mathscr{M}_{E, n, \overline{\mathscr{L}}}^{\widetilde{\mathscr{L}}}$.

(a) When the totally real field $E$ is unramified above $p$, this "naive notion" works nicely. Moreover we know from [14, Theorem 3.2.8] that the principally polarized $\left(\mathcal{O}_{E} / p \mathcal{O}_{E}\right)$-linear $\mathrm{BT}_{1}$ group $\left(A_{x}[p], \lambda_{x}[p], \iota_{x}[p]\right)$ attached to a geometric point $x$ of $\mathscr{M}_{E, n, \bar{\delta}}^{\widetilde{\mathscr{L}}}$ is determined by the $\alpha$-type of $\left(A_{x}[p], \lambda_{x}[p], \iota_{x}[p]\right)$.

(b) One difficulty when $E$ is ramified above $p$ is that the number of geometric isomorphism classes of $\left(A_{x}[p], \lambda_{x}[p], \iota_{x}[p]\right)$ may not be finite; see [1, 38.

Remark 10.6.2. For any element $l \in \mathscr{L} \cap \mathscr{L}_{\mathbb{R}}^{+}$such that $\mathscr{L}=\mathcal{O}_{E} \cdot l+p \mathscr{L}$, we have a finite etale morphism

$$
f_{l}: \mathscr{M}_{E, n, \bar{\delta}}^{\widetilde{\mathscr{L}}} \rightarrow \mathscr{A}_{g, d, n},
$$

where $d=\operatorname{card}\left(\mathscr{L} / \mathcal{O}_{E} \cdot l\right)$. A question related to Question 10.6 is to investigate basic geometric properties of the pull-back $f_{l}^{*} \mathcal{S}_{\bullet}^{\mathrm{EO}}$ of the EO stratification on $\mathscr{A}_{g, d, n}$, such as the 
dimensions, irreducible components and smoothness of strata of $f_{l}^{*} \mathcal{S}_{\bullet}^{\mathrm{EO}}$ and the incidence relation between strata.

Some of the properties of the EO stratification on $\mathscr{A}_{g, d, n}$ holds for $f_{l}^{*} \mathcal{S}_{\bullet}^{\mathrm{EO}}$. For instance, every stratum in $f_{l}^{*} \mathcal{S}_{\bullet}^{\mathrm{EO}}$ is quasi-affine. But other geometric properties of strata in $f_{l}^{*} \mathcal{S}_{\bullet}^{\mathrm{EO}}$, such as dimension, singularity and the closure relation have not been determined. The relations between $f_{l}^{*} \mathcal{S}_{\bullet}^{\mathrm{EO}}$ and other stratifications of $\mathscr{M}_{E}$, such as the congruity stratification of Lie strata and the Newton polygon stratification, await further work.

Remark 10.6.3. Satisfactory answers to Question 10.1 may very well depend on future progress on Questions 10.1(a) and 10.5. For instance if it turns out that every Lie-congruity stratum is quasi-affine and every irreducible component $\mathcal{Q}$ of $\mathcal{Q}_{\underline{c}}\left(\mathcal{N}_{\underline{e}}\right)$, the Zariski closure of $\mathcal{Q}$ is a union of irreducible components of Lie-congruity strata of $\mathscr{M}_{E}$, then one can made a good case that the Lie-congruity stratification is a good analog of the EO stratification for $\mathscr{M}_{E}$.

\section{Acknowledgments}

Chai was partially supported by NSF grant DMS1200271 and a Simons Fellowship, and he thanks Utrecht University and Academia Sinica for hospitality. Oort is grateful to the University of Pennsylvania for hospitality during many visits. Yu thanks Utrecht University for hospitality during his visit in August 2003, and acknowledges stimulating discussions with Oort which led to the proof of Theorem 9.1. He was partially supported by the MoST grants 104-2115-M-001-001-MY3 and 107-2115-M-001-001-MY2.

\section{References}

[1] F. Andreatta and E. Z. Goren, Geometry of Hilbert modular varieties over totally ramified primes, Int. Math. Res. Not. 2003 (2003), no. 33, 1786-1835.

[2] O. Blumenthal, Über Modulfunktionen von mehreren Veränderlichen, Math. Ann. 56 (1903), no. 4, 509-548.

[3] _ Über Modulfunktionen von mehreren Veränderlichen, Math. Ann. 58 (1904), no. $4,497-527$.

[4] C.-L. Chai, Every ordinary symplectic isogeny class in positive characteristic is dense in the moduli, Invent. Math. 121 (1995), no. 3, 439-479.

[5] _ _ Hecke orbits on Siegel modular varieties, in: Geometric Methods in Algebra and Number Theory, 71-107, Progr. Math. 235, Birkhäuser Boston, Boston, MA, 2005. 
[6] _ Monodromy of Hecke-invariant subvarieties, Pure Appl. Math. Q. 1 (2005), no. 2, 291-303.

[7] _ A rigidity result for p-divisible formal groups, Asian J. Math. 12 (2008), no. $2,193-202$.

[8] C.-L. Chai, B. Conrad and F. Oort, Complex Multiplication and Lifting Problems, Mathematical Surveys and Monographs 195, American Mathematical Society, Providence, RI, 2014.

[9] C.-L. Chai and F. Oort, Hecke orbits, in preparation, draft chapters available, www. math. upenn. edu/ ${ }^{\sim}$ chai.

[10] _ Hypersymmetric abelian varieties, Pure Appl. Math. Q. 2 (2006), no. 1, 1-27.

[11] _ The Hecke orbit conjecture: A survey and outlook, in: Open Problems in Arithmetic Algebraic Geometry, 235-262, Adv. Lect. Math. (ALM) 46, Int. Press, Somerville, MA, 2019.

[12] C.-L. Chai and C.-F. Yu, Fine structures and Hecke orbit problems of HilbertBlumenthal varieties, Unpublished manuscript, (2004), 31 pp.

[13] P. Deligne and G. Pappas, Singularités des espaces de modules de Hilbert, en les caractéristiques divisant le discriminant, Composition Math. 90 (1994), no. 1, 59-79.

[14] E. Z. Goren and F. Oort, Stratifications of Hilbert modular varieties, J. Algebraic Geom. 9 (2000), no. 1, 111-154.

[15] U. Görtz and M. Hoeve, Ekedahl-Oort strata and Kottwitz-Rapoport strata, J. Algebra 351 (2012), 160-174.

[16] U. Görtz and C.-F. Yu, Supersingular Kottwitz-Rapoport strata and Deligne-Lusztig varieties, J. Inst. Math. Jussieu 9 (2010), no. 2, 357-390.

[17] _ , The supersingular locus in Siegel modular varieties with Iwahori level structure, Math. Ann. 353 (2012), no. 2, 465-498.

[18] P. Hamacher, The dimension of affine Deligne-Lusztig varieties in the affine Grassmannian, Int. Math. Res. Not. IMRN 2015 (2015), no. 23, 12804-12839.

[19] - The geometry of Newton strata in the reduction modulo $p$ of Shimura varieties of PEL type, Duke Math. J. 164 (2015), no. 15, 2809-2895.

[20] P. Hamacher and E. Viehmann, Irreducible components of minuscule affine DeligneLusztig varieties, Algebra Number Theory 12 (2018), no. 7, 1611-1634. 
[21] X. He and M. Rapoport, Stratifications in the reduction of Shimura varieties, Manuscripta Math. 152 (2017), no. 3-4, 317-343.

[22] R. E. Kottwitz, Isocrystals with additional structure, Compositio Math. 56 (1985), no. 2, 201-220.

[23] _ Points on some Shimura varieties over finite fields, J. Amer. Math. Soc. 5 (1992), no. 2, 373-444.

[24] _ Isocrystals with additional structure II, Compositio Math. 109 (1997), no. 3, 255-339.

[25] R. Kottwitz and M. Rapoport, Minuscule alcoves for $\mathrm{GL}_{n}$ and $G \mathrm{Sp}_{2 n}$, Manuscripta Math. 102 (2000), no. 4, 403-428.

[26] H. Maass, Über Gruppen von hyperabelschen Transformationen, S.-B. Heidelberger Akad. Wiss. 1940 (1940), no. 2, 26 pp.

[27] B. C. Ngô and A. Genestier, Alcôves et p-rang des variétés abéliennes, Ann. Inst. Fourier (Grenoble) $\mathbf{5 2}$ (2002), no. 6, 1665-1680.

[28] F. Oort, A stratification of a moduli space of polarized abelian varieties in positive characteristic, in: Moduli of Curves and Abelian Varieties, 47-64, Aspects Math. E33, Friedr. Vieweg, Braunschweig, 1999.

[29] _ A stratification of a moduli space of abelian varieties, in: Moduli of Abelian Varieties (Texel Island, 1999), 345-416, Progr. Math. 195, Birkhäuser, Basel, 2001.

[30] Foliations in moduli spaces of abelian varieties, J. Amer. Math. Soc. 17 (2004), no. 2, 267-296.

[31] _ Minimal p-divisible groups, Ann. of Math. (2) 161 (2005), no. 2, 1021-1036.

[32] _ Foliations in moduli spaces of abelian varieties and dimension of leaves, in: Algebra, Arithmetic, and Geometry: in honor of Yu. I. Manin, Vol. II, 465-501, Progr. Math. 270, Birkhäuser Boston, Boston, MA, 2009.

[33] M. Rapoport, Compactifications de l'espace de modules de Hilbert-Blumenthal, Compositio Math. 36 (1978), no. 3, 255-335.

[34] M. Rapoport and Th. Zink, Period Spaces for p-divisible Groups, Annals of Mathematics Studies 141, Princeton University Press, Princeton, NJ, 1996.

[35] G. Van Der Geer, Hilbert Modular Surfaces, Ergebnisse der Mathematik und ihrer Grenzgebiete (3) 16, Springer-Verlag, Berlin, 1988. 
[36] E. Viehmann and T. Wedhorn, Ekedahl-Oort and Newton strata for Shimura varieties of PEL type, Math. Ann. 356 (2013), no. 4, 1493-1550.

[37] L. Xiao and X. Zhu, Cycles on Shimura varieties via geometric Satake, arXiv:1707.05700.

[38] C.-F. Yu, On reduction of Hilbert-Blumenthal varieties, Ann. Inst. Fourier (Grenoble) 53 (2003), no. 7, 2105-2154.

[39] - On the supersingular locus in Hilbert-Blumenthal 4-folds, J. Algebraic Geom. 12 (2003), no. 4, 653-698.

[40] _ Discrete Hecke orbit problems for Hilbert-Blumenthal varieties, NCTS-TPE technical report 2006-001, November 10, 2004, 20 pp.

[41] _ On the mass formula of supersingular abelian varieties with real multiplications, J. Aust. Math. Soc. 78 (2005), no. 3, 373-392.

[42] _ Irreducibility of the Hilbert-Blumenthal moduli spaces with parahoric level structure, J. Reine Angew. Math. 635 (2009), 187-211.

Chia-Fu $\mathrm{Yu}$

Institute of Mathematics, Academia Sinica and NCTS, Taipei 10617, Taiwan

E-mail address: chiafu@math.sinica.edu.tw

Ching-Li Chai

Department of Mathematics, University of Pennsylvania, Philadelphia, PA 19003, U.S.A.

E-mail address: chai@math.upenn.edu

Frans Oort

Mathematical Institute, Utrecht University, P.O. Box 80010, 3508 TA Utrecht,

Netherlands

E-mail address: f.oort@uu.nl 